\title{
Non-Equilibrium Green's Function Based Calculations of Spin Voltage in Cobalt Based Spinel Oxides
}

Anveeksh Koneru

Follow this and additional works at: https://researchrepository.wvu.edu/etd

\section{Recommended Citation}

Koneru, Anveeksh, "Non-Equilibrium Green's Function Based Calculations of Spin Voltage in Cobalt Based Spinel Oxides" (2017). Graduate Theses, Dissertations, and Problem Reports. 6004.

https://researchrepository.wvu.edu/etd/6004

This Dissertation is protected by copyright and/or related rights. It has been brought to you by the The Research Repository @ WVU with permission from the rights-holder(s). You are free to use this Dissertation in any way that is permitted by the copyright and related rights legislation that applies to your use. For other uses you must obtain permission from the rights-holder(s) directly, unless additional rights are indicated by a Creative Commons license in the record and/ or on the work itself. This Dissertation has been accepted for inclusion in WVU Graduate Theses, Dissertations, and Problem Reports collection by an authorized administrator of The Research Repository @ WVU.

For more information, please contact researchrepository@mail.wvu.edu. 


\title{
Non-Equilibrium Green's Function Based Calculations of Spin Voltage in Cobalt Based Spinel Oxides
}

\author{
Anveeksh Koneru \\ Dissertation submitted \\ to the Benjamin M. Statler College of \\ Engineering and Mineral Resources \\ at West Virginia University \\ in partial fulfillment of the requirements for the degree of \\ Doctor of Philosophy in \\ Mechanical Engineering \\ Terence Musho, Ph.D., Chair \\ David Mebane, Ph.D. \\ Aldo Romero, Ph.D. \\ Nithi T. Sivaneri, Ph.D. \\ Xueyan Song, Ph.D. \\ Nianqiang Wu, Ph.D. \\ Department of Mechanical and Aerospace Engineering \\ Morgantown, West Virginia \\ 2017
}

Keywords: Thermoelectric, Spinel, Spin Seebeck Effect, inverse spin Hall effect, ZT Copyright 2017 [Anveeksh Koneru] 


\begin{abstract}
Non-Equilibrium Green's Function Based Calculations of Spin Voltage in Cobalt Based Spinel Oxides
\end{abstract}

\author{
Anveeksh Koneru
}

Tougher sanctions on fossil fuel emissions and greater energy demand around the globe is forcing us to rely more on renewable energy resources. Clean energy technologies like thermoelectric power generation provides one solution to ease our dependence on non-renewable energy resources. Thermoelectric materials have the capability of directly converting heat to electricity. However, the efficiency of thermoelectrics has been limited due to the inherent coupling of the electronic and thermal carriers. More recently, an avenue to decouple these interactions has been experimental demonstrated using temperature gradient induced spin currents. With this new discovery comes the need for new transport models to understand and optimize their response. In providing a solution, this thesis is focused on the both the development of a 1D spin-transport model and the exploration of a spinel oxide design space comprising $\mathrm{Co}_{3} \mathrm{O}_{4}, \mathrm{Co}_{2} \mathrm{NiO}_{4}$ and $\mathrm{Co}_{2} \mathrm{ZnO}_{4}$ as the end configurations.

Ferromagnetic cobalt-based spinel oxides are potential candidates for spin-based thermoelectric energy conversion. Substitution of $\mathrm{Co}^{+2}$ and $\mathrm{Co}^{+3}$ ions with other transitional metal cations that exhibit +2 or +3 oxidation states, like $\mathrm{Ni}$ and $\mathrm{Zn}$, can affect the net spin-polarization and electronic conductivity of the material. First principles calculations using density functional theory (DFT) were performed on supercells of cobalt-based spinel oxides to obtain their electronic band structure and density of states. The band gap, electron effective mass, the Fermi energy level, and the magnetization of the material configuration were used to calculated its spin transport characteristics in the presence of a temperature gradient.

A spin driven transport model was developed that is based on a non-equilibrium Green's function (NEGF) approach. This model treats both spin channels independently and calculates electronic and spin conductivities, and spin-Seebeck coefficient of the material. The overall thermoelectric figure of merit, ZT, of the material increased when doped Ni and Zn. These trends identified a focus region around $\mathrm{Co}_{2.25} \mathrm{Ni}_{0.5} \mathrm{Zn}_{0.25} \mathrm{O}_{4}$ that has the potential for a huge spin-Seebeck enhancement. 


\section{TABLE OF CONTENTS}

Page

Chapter

LIST OF SYMBOLS . . . . . . . . . . . . . . . . . . . . xvi

1. INTRODUCTION AND LITERATURE REVIEW . . . . . . . . . 1

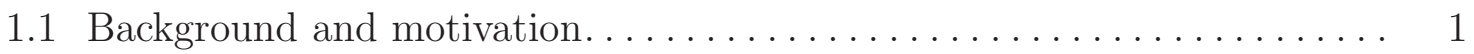

1.2 Thermoelectric energy conversion. . . . . . . . . . . . $8 \ldots \ldots$

1.2.1 Principle of operation of conventional thermoelectrics......... 8

1.2.2 Materials used for thermoelectric energy conversion applications . . 9

1.2.3 Thermal energy conversion using spin Seebeck effect.......... 13

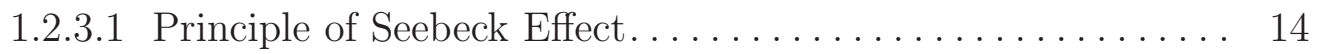

1.3 Composite Spin and Conventional Thermoelectric Generator . . . . . . . . 16

1.4 Cobalt containing spinel oxides $\ldots \ldots \ldots \ldots \ldots \ldots \ldots \ldots \ldots \ldots \ldots \ldots$

\section{COMPUTATIONAL APPROACH AND MODEL DEVELOPMENT 26}

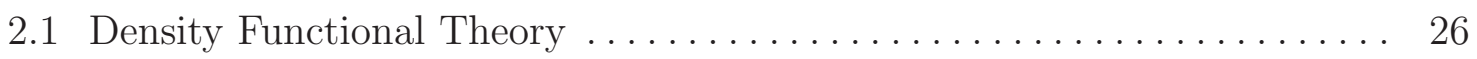

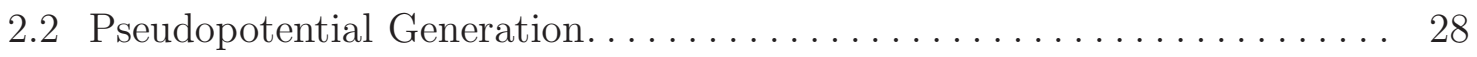

2.3 Transport Model Theory . . . . . . . . . . . . . . . . . . . . . 29

2.3.1 Non-Equilibrium Green's Function (NEGF) formalism . . . . . . . . 29

2.3.2 Calculation of the spin Seebeck coefficient............... 40

2.3 .3 Grid size and convergence .................... 41 
3. THEORY OF TRANSVERSE SPIN SEEBECK EFFECT . . . . . . 43

3.1 Spin Current Across the Magnetic and Non-Magnetic Interfaces . . . . . . 43

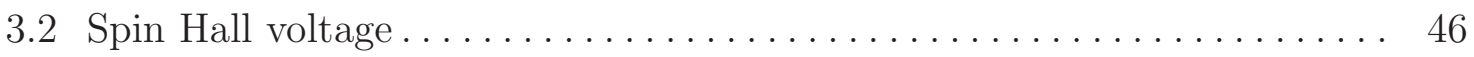

4. INVERSE SPIN HALL VOLTAGE IN LANTHANUM SUBSTITUTED YTTRIUM IRON GARNET . . . . . . . . . . . . . . . . . . 47

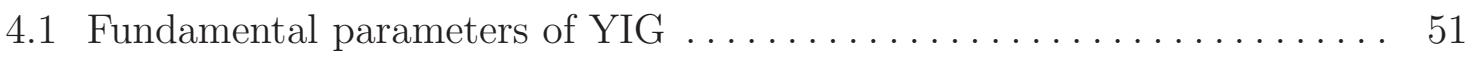

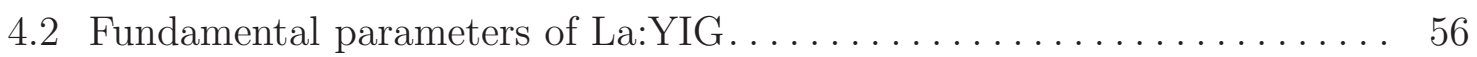

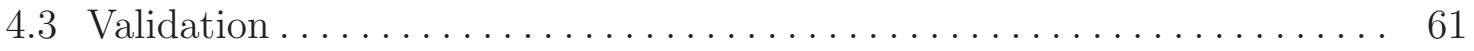

\section{EXPLORING DESIGN SPACE OF NI AND ZN SUBSTITUTED}

CO.................................. . 68

5.1 The effect of band gap and effective mass on Seebeck coefficient . . . . . 68

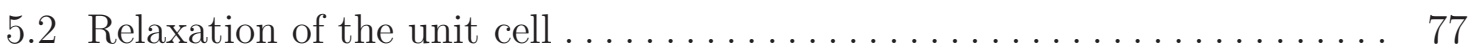

5.3 Spin polarized calculations using density functional theory . . . . . . . 78

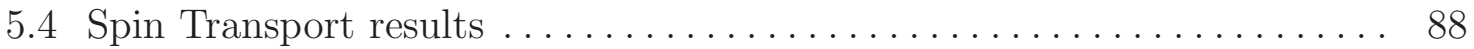

6. CONCLUSIONS . . . . . . . . . . . . . . . . . . . 97

REFERENCES. . . . . . . . . . . . . . . . . . . . . . . 99 


\section{LIST OF FIGURES}

Figure 1.1 Illustration of the energy loss in a modern internal combustion engine. Majority of this energy could potentially be recovered through waste heat recovery strategies (ex. thermoelectrics).................

Figure 1.2 Heat energy density in microprocessors linearly increases with the number of transistors. Its power density is estimated to be more than that of a nuclear reactor $[5] \ldots \ldots \ldots \ldots \ldots \ldots \ldots \ldots \ldots \ldots \ldots \ldots \ldots \ldots$

Figure 1.3 Efficiency of a TE device in the presence of different temperature bias. Each curve is specified with the respective $\mathrm{zT}$ from 1.0 to $10 . \ldots \ldots \ldots$

Figure 1.4 Timeline for thermoelectric material performance from 1990 to present. There has been a plateau of ZT since 2000's when nano-modification were implemented. To further increase the figure of merit new strategies such has spin caloric thermoelectrics are necessary...........

Figure 1.5 Conventional Thermoelectric generator. The series connection of p-type and n-type materials uses the temperature difference across the two

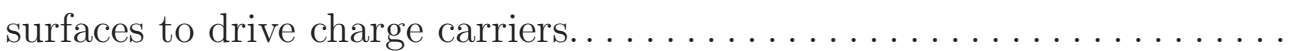

Figure 1.6 Spin Seebeck Effect. A net spin flux enters the metal contact $(\mathrm{Pt})$ when connected on top of a ferromagnetic material that is placed under a net thermal bias. The net spin causes results in a voltage $(\mathrm{V})$ across the two ends of Pt metal contact. ............................ 15 
Figure 1.7 Spin voltage induced into Pt contacts at various locations along the La:YIG device [67]. There is a linear dependence on the voltage and position along the device........................ 16

Figure 1.8 Top figure is a longitudinal configuration where the spin contact is perpendicular to the magnetic polarization. The bottom figure is a transverse configuration (configuration studied within) and is configured when a Pt metal contact is placed on the ferromagnet such that spin current is perpendicular to the heat gradient........... 17

Figure 1.9 Top is an isometric view of the combined conventional and spin based thermoelectric material. Bottom is an enlarged view of the thermoelectric generator showing the Pt metal contacts (in red color) attached on the transverse direction to the p-type and n-type thermoelectric legs. These Pt contacts generate additional spin voltage which can be combined with the conventional charge voltage to result in an increase of the overall ZT of the material. . . . . . . . . . . 19

Figure 1.10 A super cell of a 2x2x1 primitative unit cells that is based on a spinel structure comprising 56 atoms. The silver balls represent tetrahedral sites which have coordination number 4 , the blue balls represent octahedral sites which have coordination number 6 and the red balls represent oxygen atoms. The difference in spin between the tetrahedral and octahedral sub lattice allows control of the ferrimagnetic properties of the structure. $\ldots \ldots \ldots \ldots \ldots \ldots \ldots \ldots \ldots \ldots \ldots \ldots \ldots \ldots \ldots \ldots \ldots$

Figure 1.11 Compositional space comprising $\mathrm{Co}_{3} \mathrm{O}_{4}, \mathrm{Co}_{2} \mathrm{ZnO}_{4}$ and $\mathrm{Co}_{2} \mathrm{NiO}_{4}$ as end points. . . . . . . . . . . . . . . . . 
Figure 1.12 Crystal field splitting of $\mathrm{Ni}^{+2}, \mathrm{Ni}^{+3}, \mathrm{Co}^{+2}$ and $\mathrm{Co}^{+3}$ configurations.....

Figure 2.1 A three probe system consisting of left electrode with temperature $\mathrm{T}_{H}$, right electrode with temperature $\mathrm{T}_{C}$ and an ISHE electrode on the scattering region to measure the amount of current from the scattering region into the electrode. . . . . . . . . . . . . . . . .

Figure 2.2 Description of the scattering region divided into $\mathrm{N}$ lattice points. The number of grid points $\mathrm{N}$ depends on length of the lattice.......... 32

Figure 2.3 NEGF flow chart of the self-consistent method. The self-consistent criteria is when consecutive energy difference reaches below $1 \mathrm{e}-6 . \ldots \ldots$.

Figure 2.4 An illustration of density of states for spin-polarized ferromagnetic a) Metal b) Semiconductor. The electrochemical potential of the spin-up conduction electrons, $\mu_{\uparrow}$, is different from that of spin-down conduction electrons, $\mu_{\downarrow}$. The difference in the energy gap for spin-up(spin-down)

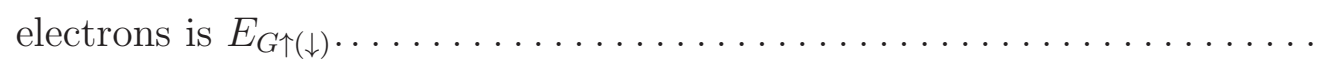

Figure 2.5 Varying $V_{I S H E}$ to neutralize spin-up and spin-down currents separately in the scattering region, gives the respective spin Seebeck coefficients

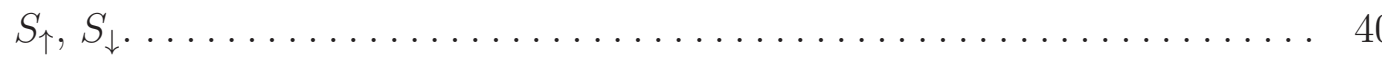

Figure 2.6 Increasing the grid size to see variation in the population density in the scattering region, given in blue color. For each grid point the respective computational time consumed is shown in red.

Figure 4.1 Experimental demonstration of spin seebeck effect in La:YIG at various

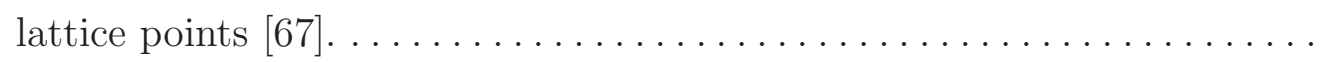
48 
Figure 4.2 Spin voltage induced into Pt contacts at various locations along the

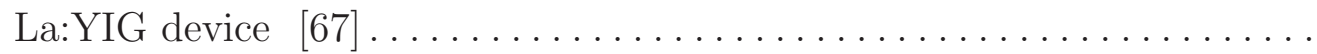

Figure 4.3 A 80 atom super cell of YIG. The green atoms represent $\mathrm{Fe}^{+3}$ ions, the gray atoms represent $\mathrm{Y}^{+3}$ ions and the red atoms represent $\mathrm{O}^{-2}$ ions. . . 50

Figure 4.4 The atom projected density of states of iron, yttrium and oxygen show their contributions towards the total density of states near the Fermi region in the YIG material lattice. The positive density of states represent the contribution due to spin up electrons and the negative density of states represent the contribution due to spin down electrons... 51

Figure 4.5 The spin up channel of YIG material $\ldots \ldots \ldots \ldots \ldots \ldots \ldots \ldots \ldots$

Figure 4.6 The spin down channel of YIG material $\ldots \ldots \ldots \ldots \ldots \ldots \ldots \ldots \ldots$

Figure 4.7 The valence band corresponds to the spin up channel and the conduction band corresponds to the spin down channel of YIG material.. 54

Figure 4.8 Effective mass of spin down conduction band of YIG in $\mathrm{X}$ to $\Gamma$ direction, $\left(\mathrm{m}_{x}\right)$. The blue color curve shows the fit of effective mass using the Equation 4.1 with a value of $\mathrm{m}_{x}=0.4821^{*} \mathrm{~m}_{e} \ldots \ldots \ldots \ldots \ldots$

Figure 4.9 Effective mass of spin down conduction band of YIG in $\Gamma$ to $\mathrm{L}$ direction, $\left(\mathrm{m}_{y}\right)$. The blue color curve shows the fit of effective mass using the Equation 4.2 with a value of $\mathrm{m}_{y}=0.5317^{*} \mathrm{~m}_{e} \ldots \ldots \ldots \ldots \ldots$

Figure 4.10 Effective mass of spin down conduction band of YIG in $\Gamma$ to $\eta$ direction, $\left(\mathrm{m}_{z}\right)$. The blue color curve shows the fit of effective mass using the Equation 4.3 with a value of $\mathrm{m}_{z}=0.5048^{*} \mathrm{~m}_{e} \ldots \ldots \ldots \ldots \ldots$. 57 
Figure 4.11 A 80 atom super cell of La:YIG. The green atoms represent $\mathrm{Fe}^{+3}$ ions, the gray atoms represent $\mathrm{Y}^{+3}$ ions and the red atoms represent $\mathrm{O}^{-2}$ ions. The four purple atoms in La:YIG super cell that replace the $\mathrm{Y}^{+3}$ ions represent $\mathrm{La}^{+3}$ ions. The positive density of states represent the contribution due to spin up electrons and the negative density of states represent the contribution due to spin down electrons........... 58

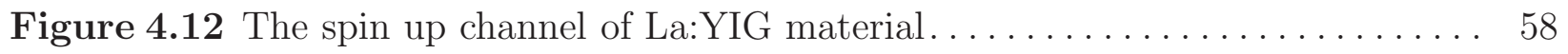

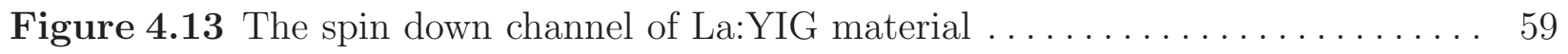

Figure 4.14 The valence band corresponds to the spin up channel and the conduction band corresponds to the spin down channel of La:YIG

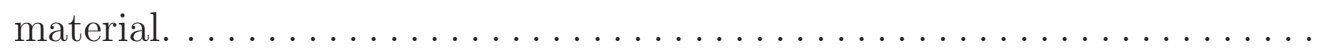

Figure 4.15 Effective mass of spin down conduction band of La:YIG in X to $\Gamma$ direction, $\left(\mathrm{m}_{x}\right)$. The blue color curve shows the fit of effective mass using the Equation 4.1 with a value of $\mathrm{m}_{x}=0.4462^{*} \mathrm{~m}_{e} \ldots \ldots \ldots \ldots$

Figure 4.16 Effective mass of spin down conduction band of La:YIG in $\Gamma$ to $\mathrm{L}$ direction, $\left(\mathrm{m}_{y}\right)$. The blue color curve shows the fit of effective mass using the Equation 4.2 with a value of $\mathrm{m}_{y}=0.4898^{*} \mathrm{~m}_{e} \ldots \ldots \ldots \ldots 1$

Figure 4.17 Effective mass of spin down conduction band of La:YIG in $\Gamma$ to $\eta$ direction, $\left(\mathrm{m}_{z}\right)$. The blue color curve shows the fit of effective mass using the Equation 4.3 with a value of $\mathrm{m}_{z}=0.4577^{*} \mathrm{~m}_{e} \ldots \ldots \ldots \ldots$

Figure 4.18 The atom projected density of states of iron, lanthanum, yttrium and oxygen show their contributions towards the total density of states near the Fermi region in the La:YIG material lattice. . . . . . . . . . 
Figure 4.19 Description of the scattering region divided into $\mathrm{N}$ lattice points. The number of grid points $\mathrm{N}$ depends on the length of the lattice. . . . . . . . 64

Figure 4.20 Validation of the model with the experimental data available for La:YIG. The $V_{I S H E}$ voltage in $\mu \mathrm{V}$ is calculated at the different points on the material lattice along the $\mathrm{x}$-direction. . . . . . . . . . .

Figure 5.1 The variation of current density generated in the scattering channel with band gap and effective mass of conduction electrons in the scattering channel. The temperature difference between the end contacts was $20 \mathrm{~K}$. The $\mathrm{x}$-axis represents the band gap in the material, $\mathrm{y}$-axis represents $\frac{\text { effectivemass }}{\text { massofelectron }}$ and z-axis represents the current density in the scattering channel on a log scale. An increase in the band gap causes an exponential decrease in the current density. An increase in effective mass causes a linear decrease in the cirrent density. . . . . . . . .

Figure 5.2 The variation of electrical conductivity $(\sigma,($ siemen $/ m))$ in the scattering region with band gap and effective mass of conduction electrons in the scattering channel. The temperature difference between the end contacts was $20 \mathrm{~K}$. The $\mathrm{x}$-axis represents the band gap in the material, y-axis represents $\frac{\text { effectivemass }}{\text { massofelectron }}$ and z-axis represents the electrical conductivity in a log scale. $\sigma$ decreases exponentially with an

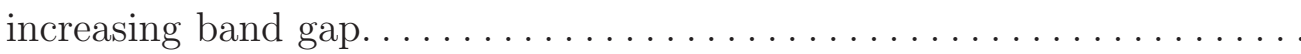


Figure 5.3 The variation of conventional Seebeck coefficient, in $\mu \mathrm{V} / \mathrm{K}$, with band gap and effective mass of conduction electrons in the scattering channel. The temperature difference between the end contacts was 20K. The $\mathrm{x}$-axis represents the band gap in the material, $\mathrm{y}$-axis represents $\frac{\text { effectivemass }}{\text { massofelectron }}$ and z-axis represents the conventional Seebeck coefficient. Higher band gap materials have high Seebeck coefficient. . . . 72

Figure 5.4 The variation of power factor, in $\mu \mathrm{W} / \mathrm{mK}^{2}$, with band gap and effective mass of conduction electrons in the scattering channel. The temperature difference between the end contacts was $20 \mathrm{~K}$. The $\mathrm{x}$-axis represents the band gap in the material, y-axis represents $\frac{\text { effectivemass }}{\text { massofelectron }}$ and $\mathrm{z}$-axis represents the power factor of the material in log scale. . . . 73

Figure 5.5 The variation of power factor, in $\mu \mathrm{W} / \mathrm{mK}^{2}$, with band gap and effective mass of conduction electrons in the scattering channel. The temperature difference between the end contacts was $20 \mathrm{~K}$. The $\mathrm{x}$-axis represents the band gap in the material, y-axis represents $\frac{\text { effectivemass }}{\text { massofelectron }}$ and $\mathrm{z}$-axis represents the power factor of the material in log scale. . . . 74

Figure 5.6 The variation of electronic contribution of thermal conductivity, $\kappa_{e}$ in $\mathrm{W} / \mathrm{mK}$, with variation in band gap and effective mass of conduction electrons in the scattering channel. The temperature difference between the end contacts was $20 \mathrm{~K}$. The x-axis represents the band gap in the material, y-axis represents $\frac{\text { effectivemass }}{\text { massofelectron }}$ and z-axis represents the $\kappa_{e}$ of the material. The low band gap materials have high electronic contribution towards the thermal conductivity compmared to the low

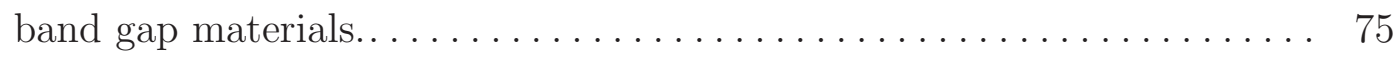


Figure 5.7 The variation of thermoelectric performance, ZT, with variation in band gap and effective mass of conduction electrons in the scattering channel. The temperature difference between the end contacts was 20K. The $\mathrm{x}$-axis represents the band gap in the material, $\mathrm{y}$-axis represents $\frac{\text { effectivemass }}{\text { massofelectron }}$ and z-axis represents the $\kappa_{e}$ of the material. The intermediate region having the band gaps around $0.14 \mathrm{eV}$ to $0.35 \mathrm{eV}$ seem to posess a ZT with a value of 0.5. Low band gap materials have high electronic thermal conductivity took a toll on their thermoelectric

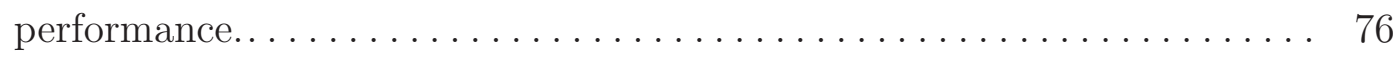

Figure 5.8 The atom projected density of states in $\mathrm{Co}_{3} \mathrm{O}_{4}$ spinel lattice. The Co atoms in the tetrahedral (tet) sites majorly contribute to the net spin polarization in the material due to their difference in the energy states while Co atoms in the Octahedral (oct) sites have minimal contribution

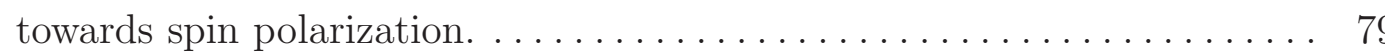

Figure 5.9 Atom projected density of states for $\mathrm{Co}_{2.5} \mathrm{Zn}_{0.5} \mathrm{O}_{4}$. Blue lines indicate the states due to cobalt atoms in the tetrahedral states that majorly influence the spin polarization and conductivity in a spinel matrix. . . . 80

Figure 5.10 Crystal field splitting of $\mathrm{Zn}^{+2}$ in tetrahedral occupancy. . . . . . . 81

Figure 5.11 Density of states for $\mathrm{Co}_{2} \mathrm{ZnO}_{4}$. This spinel configuration shows no net spin polarization due to zero contribution from completely filled $\mathrm{Zn}^{+2}$ atoms occupying tetrahedral sites. . . . . . . . . . . . . .

Figure 5.12 Density of states for $\mathrm{Co}_{2} \mathrm{ZnO}_{4}$. This spinel configuration shows no net spin polarization due to zero contribution from completely filled $\mathrm{Zn}^{+2}$ atoms occupying tetrahedral sites. . . . . . . . . . . . . . . . . 
Figure 5.13 Electron density plot of the $\mathrm{Co}_{2} \mathrm{Ni}_{0.25} \mathrm{Zn}_{0.75} \mathrm{O}_{4}$ showing electron cloud localised around $\mathrm{Ni}$ atoms on the tetrahedral sites. The energy range within $0.25 \mathrm{eV}$ associates the localised energy states only to $\mathrm{Ni}$ atoms which donot form a unifom interlink through out the cell and hence they cannot contribute to electronic conduction within the cell.........

Figure 5.14 A ternary plot of spin up and spin down band gaps of different configurations in the $\operatorname{design}$ space. $\ldots \ldots \ldots \ldots \ldots \ldots \ldots \ldots \ldots$

Figure 5.15 Spin up conduction and valance bands of $\mathrm{Co}_{3} \mathrm{O}_{4}$. The three effective mass $\mathrm{m}_{x}, \mathrm{~m}_{y}$ and $\mathrm{m}_{z}$ are calculated for three paths $\mathrm{X}$ to Gamma, $\Gamma$ to $\mathrm{L}$ and $\Gamma$ to $\mathrm{N}$ respectively. The calculated effective mass curves are plotted on the conduction band. This band structure plot gives the information about the band gap between conduction and valance band edges and the three effective mass.................. 86

Figure 5.16 Current in $\mathrm{A} / \mathrm{m}^{2}$ for the configuraion space. The $\mathrm{x}$-axis shows

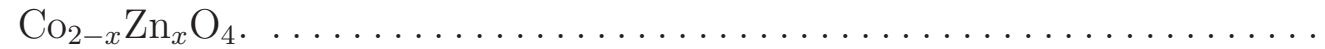

Figure 5.17 Electronic conductivity of the cobalt spinels, in Seimen/m for the configuraion space. High mobility associated with Ni substituted spinels has a high electronic conductivity and high band gap of Zn substituted cobalt spinels have low electronic conductivity. . . . . . . . . . 90

Figure 5.18 Electronic thermal conductivity of the cobalt spinels, in Seimen/m for the configuraion space. High mobility associated with Ni substituted spinels has a high electronic conductivity and electron contribution to thermal conductivity. $\ldots \ldots \ldots \ldots \ldots \ldots \ldots \ldots \ldots \ldots \ldots \ldots \ldots \ldots \ldots$ 
Figure 5.19 The Seebeck coefficient is proportional to the band gap in the material. Higer band gap associated to Zn substituted spinel shows a high Seebeck coefficient. Ni substituted cobalt spinel has the least band gap and hence has a low Seebeck coefficient. .................... 92

Figure 5.20 The power factor of the spinel configuration space. Power factor is directly proportional to the electrical conductivity of the material. Here, Ni substitution in the lattice showed the highest electrical conductivity and hence the power factor is highest for $\mathrm{Ni}$ occupation in tetrahedral sittes. . . . . . . . . . . . . . . . . . . . . . . 93

Figure 5.21 The thermoelectric performance of spinel oxides. The end configurations have a maximum $\mathrm{ZT}$ value of 0.15 , but the intermediate configurations having $\mathrm{Ni}$ and Zn substituted in the spinel lattice show an increase in the ZT to a value $0.3 \ldots \ldots \ldots \ldots \ldots \ldots \ldots \ldots \ldots \ldots \ldots$

Figure 5.22 The total voltage output from the cell increases as the Seebeck coefficient increases. The inverse spin Hall voltage is improved due to the magnetization and the increase in the difference between the two

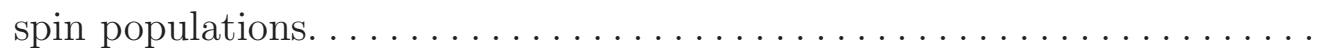

Figure 5.23 Spin Figure of merit of the configuraion space. Including the inverse spin Hall voltage, that is predominant in Ni substitution improved the $\mathrm{ZT}$ of $\mathrm{Ni}$ from 0.05 to 0.3 , which is almost 1 fold increase in ZT....... 96 


\section{LIST OF TABLES}

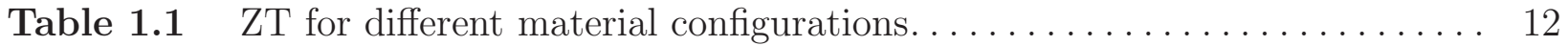

Table 1.2 Typical Cost per Kg of different material configurations [29] [57] . . . . 13

Table 4.1 Parameters used in the experimental setup as reported in literature [67] and the parameters used in verification. ............... 64

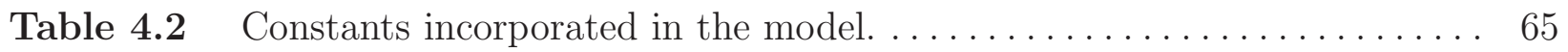

Table 5.1 The effect of Hubbard U parameter on the band gap and effective mass spin down conduction electrons of La:YIG material............ 68

Table 5.2 The band gap, effective mass of spin up and spin down conduction channels and, the magnetic moment of each configuration along the

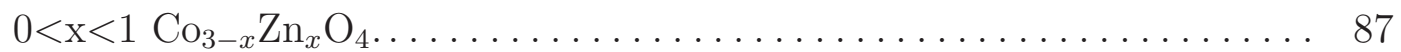

Table 5.3 The band gap, effective mass of spin up and spin down conduction channels and, the magnetic moment of each configuration along the

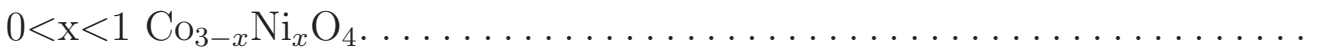

Table 5.4 The band gap, effective mass of spin up and spin down conduction channels and, the magnetic moment of each configuration along the

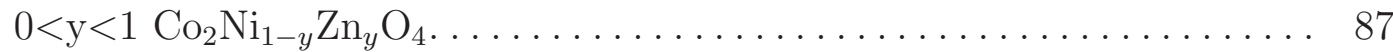




\section{LIST OF SYMBOLS}

$\eta_{\max }=$ Maximum efficiency

$\theta=$ Spin Hall angle of Platinum metal

$\mathrm{TW}=$ Terawatt

$\mathrm{TE}=$ Thermoelectric

$\mathrm{ZT}=$ Thermoelectric figure of merit

$\mathrm{S}=$ Seebeck coefficient

$\mathrm{S}_{\uparrow}=$ Spin up Seebeck coefficient

$\mathrm{S}_{\downarrow}=$ Spin down Seebeck coefficient

$\kappa=$ Total thermal conductivity

$\kappa_{e}=$ Electronic thermal conductivity

$\kappa_{p}=$ Phonon thermal conductivity

$\sigma=$ Electrical conductivity

$\mathrm{T}_{c}=$ Temperature of cold contact

$\mathrm{T}_{h}=$ Temperature of hot contact

$\mathrm{T}_{F}=$ Temperature of magnons in ferromagnetic material

$\mathrm{T}_{N}=$ Temperature of non-magnetic material

$\mathrm{L}=$ Lorentz number

$\mathrm{E}=$ Energy

$\mathrm{E}_{e m f}=$ electromotive force in $\mathrm{eV}$

$\mathrm{J}_{s}=$ Net spin current

$\mathrm{J}_{f l}=$ spin fluctuation current

$\mathrm{PV}=$ Photovoltaic

$\Delta \mathrm{T}=$ difference in temperature between hot and cold contacts

$\mathrm{NM}=$ Non-magnetic metal 
$\mathrm{FM}=$ Ferromagnetic material

$\mathrm{H}=$ Hamiltonian

$\mathrm{H}_{o}=$ External magnetic field

$\mathrm{V}_{I S H E}=$ Inverse spin Hall voltage

$\hbar=$ Reduced Plank's constant

$\mathrm{M}_{s}=$ Saturation magnetization

$\mathrm{DFT}=$ Density functional theory

NEGF $=$ Non-Equilibrium Green's Function

$f_{l(r)}=$ Fermi-Dirac distribution function

$\Xi=$ Transmission function

$\epsilon=$ Energy level

$\mu=$ Electrochemical potential

$E_{c}=$ Conduction band edge

$m^{*}=$ Effective mass

$m_{\downarrow}^{*}=$ Effective mass of spin down electrons

$m_{\uparrow}^{*}=$ Effective mass of spin up electrons

$\chi=(0,0,1 / 2) \mathrm{k}$-point

$\Gamma=(0,0,0) \mathrm{k}$-point

$\eta=(0,-1 / 2,1 / 2) \mathrm{k}$-point

$E_{c x}=$ Conduction band edge in $\chi$ to $\Gamma$ direction

$E_{c y}=$ Conduction band edge in $\Gamma$ to $(1 / 2,0,0)$ direction

$E_{c z}=$ Conduction band edge $\Gamma$ to $\eta$ direction

$g_{r}=$ Real part of spin mixing conductance

$\boldsymbol{m}=$ unit vector parallel to the magnetization in the material

$\dot{\boldsymbol{m}}=$ Rate of change of magnetization

$\mathrm{V}=$ Volume of the NM metal

$\alpha^{\prime}=$ Damping enhancement due to spin pumping 
$H_{e f f}=$ Effective external magnetic field

$\omega=$ Frequency

$\omega_{o}=$ Ferromagnetic resonance frequency

$\chi($ omega $)=$ transverse dynamic susceptibility matrix

$\boldsymbol{h}^{\prime}=$ Resultant magnetic field acting on magnetization

$\gamma=$ Electron gyro-magnetic ratio

$L_{s}^{\prime}=$ Interfacial Seebeck coefficient

$\rho=$ Electrical resistivity

$l=$ Length of non-magnetic metal contact

$\psi=$ Many particle wave function

$\Sigma^{\text {out }}=$ Energy outflow

$\Sigma^{i n}=$ Energy inflow

$\Sigma_{l}^{\text {out }}=$ Energy outflow at the left contact

$\sum_{r}^{\text {out }}=$ Energy outflow at the right contact

$\{s\}=$ Inflow energy from source

$[G]=$ Green's Function

$G^{n}=$ Electron density

$\Gamma_{l}=\mathrm{NxN}$ anti-Hermitian matrix of $\Sigma_{l}^{\text {out }}$

$\Gamma_{r}=\mathrm{NxN}$ anti-Hermitian matrix of $\sum_{r}^{\text {out }}$

$\mathrm{a}=$ distance between cell centers

$E_{H}=$ Hartree Energy

$T_{s}=$ Kinetic energy

$E_{x c}=$ Exchange-correlation energy functional

$V_{x c}=$ Exchange-correlation potential

LSDA $=$ Local spin density approximation

GGA $=$ Generalised gradient approximation

$\mathrm{LDA}=$ Local density approximation 
$E_{x c}^{L D A}=$ Exchange-correlation energy functional using LDA

$\mathrm{PBE}=$ Perdew-Burke-Ernzerhof

$\mathrm{GGA}+\mathrm{U}=$ Generalised gradient approximation + Hubbard $\mathrm{U}$ parameter 


\section{CHAPTER 1}

\section{INTRODUCTION AND LITERATURE REVIEW}

\subsection{Background and motivation}

It is estimated that over $90 \%$ of worlds electricity which is generated from heat engines, typically operate with 30-40\% efficiency [1]. If we look at energy distribution in internal combustion engines, represented in Figure 1.1, approximately $40 \%$ of the fuel energy is emitted as exhaust gas, 30\% is dissipated through engine coolant, $5 \%$ is lost as radiation and friction, only $25 \%$ is available for vehicle mobility and to power some on-board accessories [2],[3]. It has been reported that, US manufacturing sector alone loses more than $3000 \mathrm{TW}$ of energy as waste heat, an energy equivalent to 1.72 billion barrels of oil [4]. If the current rate of oil usage for energy production is continued, the reserves are bound to deplete and emissions will pose a greater threat to human life.

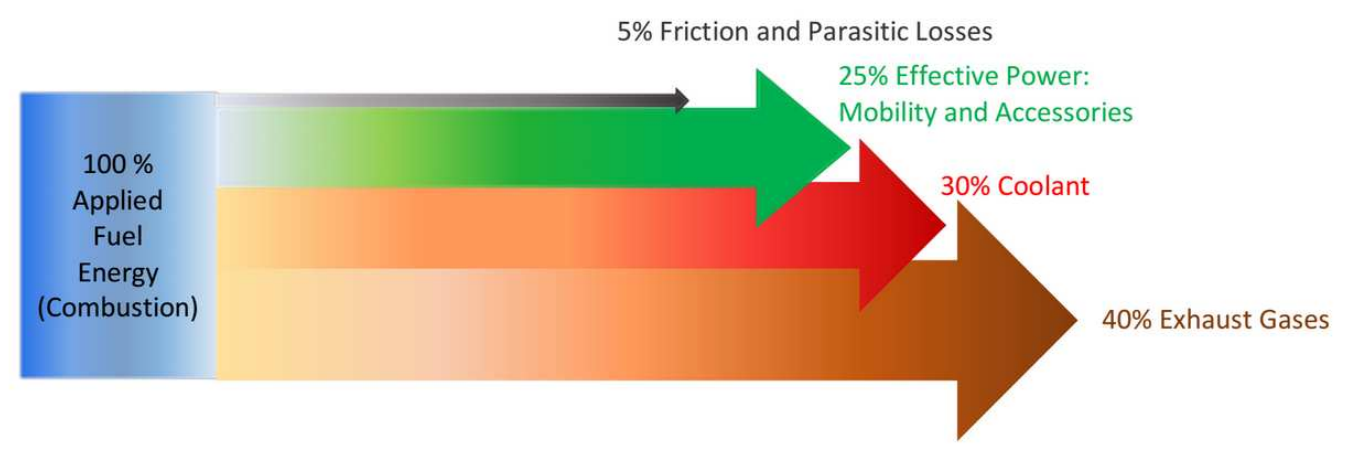

Figure 1.1 Illustration of the energy loss in a modern internal combustion engine. Majority of this energy could potentially be recovered through waste heat recovery strategies (ex. thermoelectrics). 
Even in electronics, a huge amount of heat energy is released from microprocessors, whose heat energy density is more than that of a nuclear reactor. This has been depicted by University of Texas research scientists as shown in Figure 1.2 [5]. With the increase in the number of transistors per unit area, the power density measured in $\mathrm{W} / \mathrm{cm}^{2}$ is more than that of a Nozzle in a rocket. In order to protect the processors from such high heat during high processing rates, an on board dissipation system is installed for better dissipation.

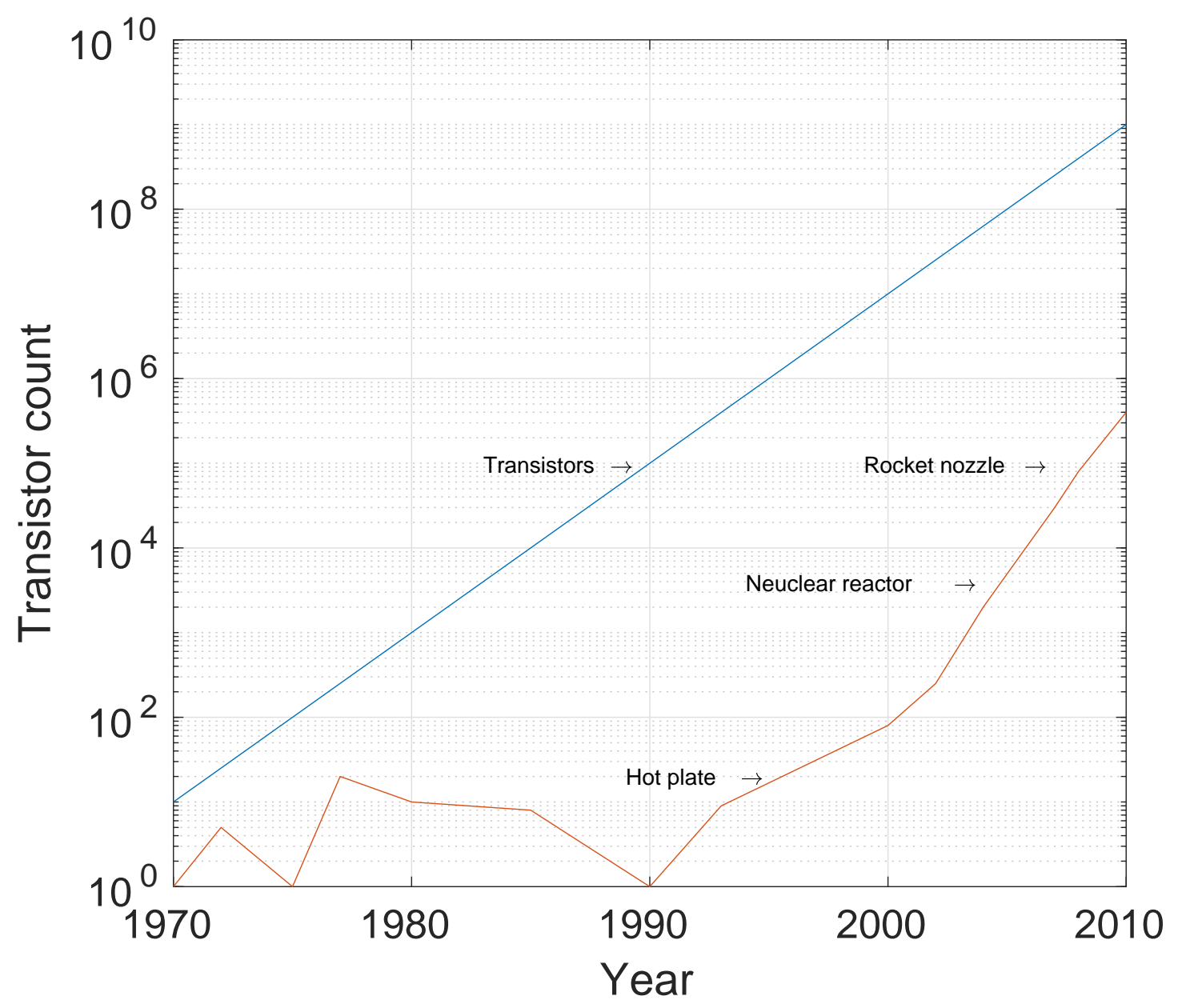

Figure 1.2 Heat energy density in microprocessors linearly increases with the number of transistors. Its power density is estimated to be more than that of a nuclear reactor [5].

In heat engines and microprocessors a tremendous amount of energy is lost to waste 
heat. Different ways of heat conversion technologies have be incorporated to convert waste heat back to electricity. Several example of heat conversion technologies in organic Rankin cycles (ORC) [6, 7], thermionic [8, 9], and thermoelectrics. The latter case being the focus of this research with all having the potential to diminish green house gas emissions while also improving the overall cycle efficiency. Thermoelectric (TE) energy conversion is one of the most promising way to convert low grade waste heat directly into electricity. While TEs are a promising technology to answer several of the proposed questions there still a need to improve the efficiency of the devices. As the technology stands currently there is a fundamental barrier that is just now being surpassed that has limited the efficiency of said devices. The technology barrier is now being surpassed by taking a new approach that uses an underlying principle of the spin Hall effect. This requires taking a new perspective on how the charge carriers transport through the material and determining what materials are optimal for such operation.

The performance of conventional thermoelectric materials that generate energy from fermionic transport can be calculated by their thermoelectric figure of merit, ZT, given by Equation 1.1. Higher the ZT of a TE material, higher will be its capability to convert waste heat to electricity. To improve the ZT, a material must embody a high electronic conductivity while simultaneously being a poor thermal conductor. This can be done by increasing the numerator in the ZT equation, the power factor $S^{2} \sigma$, and simultaneously decreasing the value of the denominator, the thermal conductivity $(\kappa)$. But according to Wiedemann-Franz law, given in Equation 1.2, the ratio of electronic contribution of thermal conductivity, $\kappa$, to the electrical conductivity $(\sigma)$ at any particular temperature is a constant, equal to Lorentz number. This equation equates that the electric and thermal conductivities are proportional and there is a physical limit. Granted the Wiedemann-Franz law is only valid for metallic based materials, this relation is only slightly modified or semiconducting material. That being said, the Wiedeman-Franz law presents a physical relation that is extremely challeng- 
ing to bypass and therefore the ZT of bulk and nano-modified materials has not realized a significant improvement.

The figure of merit for both conventional and spin based thermoelectrics is given by the following expression,

$$
Z T=S^{2} \sigma T / \kappa
$$

where $\mathrm{S}$ is Seebeck coefficient, $\sigma$ is electrical conductivity, $\mathrm{T}$ is the temperature and $\kappa$ is thermal conductivity which includes electron and phonon contribution to thermal conductivity.

Similarly, the Wiedemann-Franz law is given by,

$$
\kappa / \sigma=L T
$$

where L Lorentz number (for metals $\mathrm{L}$ is $2.44^{*} 10^{-8} \mathrm{~W} \Omega \mathrm{K}^{-2}$ ) and $\mathrm{T}$ is temperature.

It should be noted that the Wiedmann-Franz law is no longer valid as depending on the spin channel configuration the spin transport carriers are not traveling in the transverse direction relative to the thermal gradient.

As performance of most engineering appliances is compared with their respective efficiencies, the performance of a TE material can also be represented in terms of efficiency using the Equation 1.3. A TE device with different ZT and under different temperature bias, at $300 \mathrm{~K}$ operational temperature is provided in the Figure 1.3. To be able to compete with heat engines, a TE material must have an efficiency greater than $20 \%$. For instance a material having $\mathrm{ZT}=3.0$, present in a $300 \mathrm{~K}$ temperature bias at $300 \mathrm{~K}$ operating temperature will show an efficiency 20\%. No material has yet achieved a ZT>3 [10]. Most commercial TE modules operate at a ZT value below 1 and at temperature bias less than $200 \mathrm{~K}$ which 
means less than $10 \%$ operational efficiency.

$$
\eta_{\max }=\frac{T_{h}-T_{c}}{T_{h}} \frac{\sqrt{1+Z T}-1}{\sqrt{1+Z T}+\frac{T_{c}}{T_{h}}}
$$

Here, $T_{h}$ is the temperature of the hot surface, $T_{c}$ is the temperature of the cold surface, $z T$ is the thermoelectric figure of merit for the device under $T_{h}-T_{c}$ temperature gradient and $\eta_{\max }$ is the efficiency of the device.

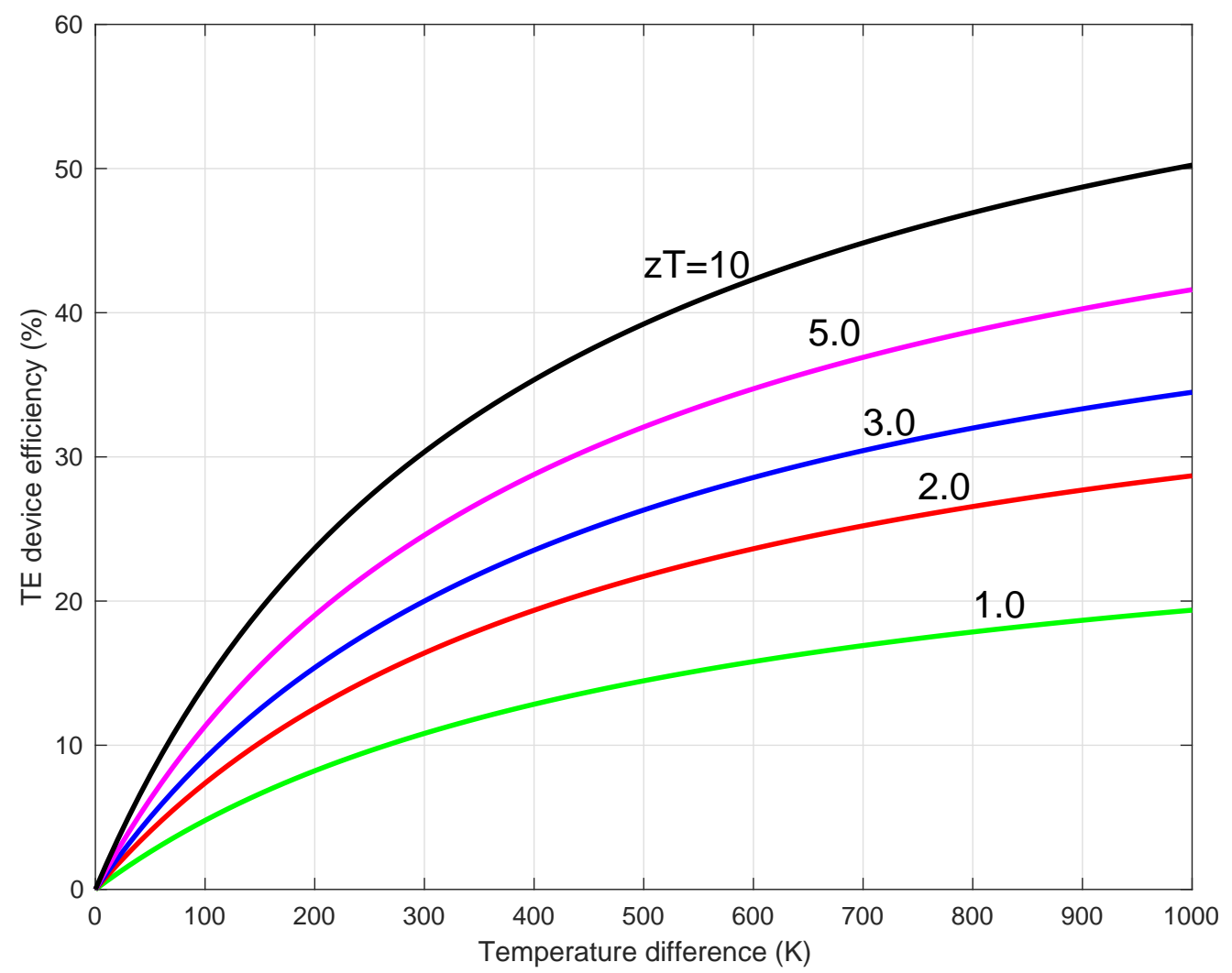

Figure 1.3 Efficiency of a TE device in the presence of different temperature bias. Each curve is specified with the respective zT from 1.0 to 10 .

Among the alternative energy sources like sun, wind, geothermal and tidal; PV and TE technologies use the energy received from Sun and are competing with the traditional 
heat engines. The efficiency of PV technologies range between $8 \%$ and $20 \%$ [11] [12], TE technologies operate between $5 \%$ and $10 \%$ efficiencies [13] [14], where as Rankine cycle heat engines operate near $42 \%$ efficiency. The cost per kilo watt hour (12-30 cents per Kwh of PV [15] compared to 2.5 to 6 cents per Kwh for coal fired power plants). Current solar based PV system utilizes the photon energy present in light to generate electric energy. But the heat part of sun light goes waste during the photon energy conversion to voltage. So, proper incorporation of TE materials near the heat source can to convert the vented heat energy to electricity thereby improving the overall performance of the system. Currently available commercial thermoelectric materials based of telluride material like $\mathrm{PbTe}, \mathrm{Bi}_{2} \mathrm{Te}_{3}$ [16] which operate in a temperature range $250^{\circ} \mathrm{C}$ to $500^{\circ} \mathrm{C}$ are highly toxic in nature and expensive. Hence, Te based thermoelectrics are mainly used for spacecraft applications. For a wide range of commercial applications their performance has to be moderatly improved through nano-modification and diminishing the lattice contribution to the figure of merit, ZT given in Equation 1.1.

The current status of thermoelectric materials is shown in Figure 1.4. From early 1990 's to the early 2000's the ZT of the material has been around 1, while from the late 2000's to the early 2010's saw an improvement in the ZT to 2 due to the effect of reduced dimensionality on improving material properties. Currently there are reports of materials reaching a ZT of 2.6 [17]. With the advent in theoretical insight along with advancements in nano material fabrication and processing technologies, thermoelectric materials with improved ZT $(>3)$ are expected in the next 5-10 years.

Energy conversion technologies have always been an intergral part of energy research. In the recent years, thermal gradient driven spin transport has gained momentum due to its wide scope in solid state electronics to convert waste heat into useful form of energy [18]. Though the anamolous Hall effect, which is the underlying mechanism in spin transport, 


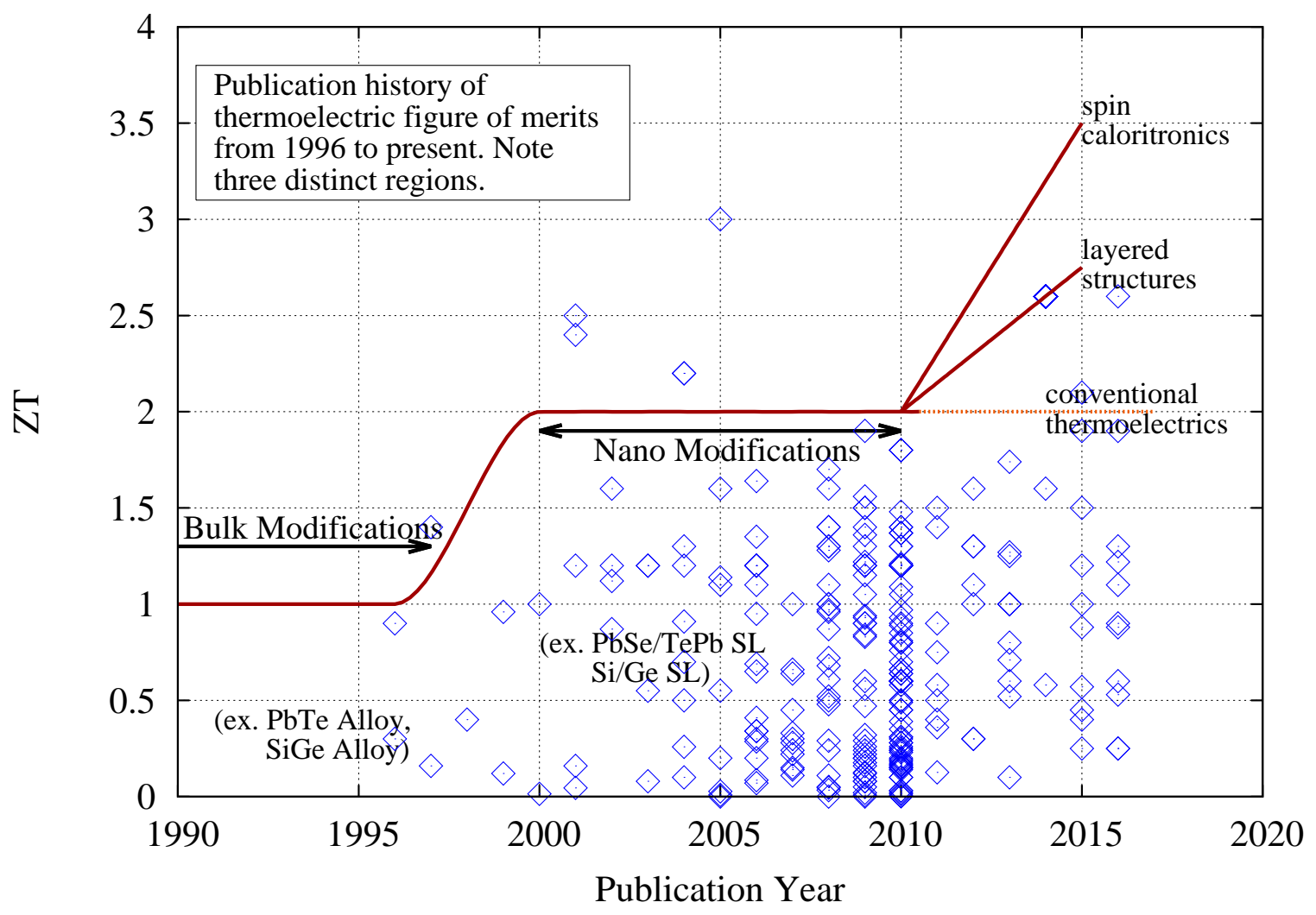

Figure 1.4 Timeline for thermoelectric material performance from 1990 to present. There has been a plateau of ZT since 2000's when nano-modification were implemented. To further increase the figure of merit new strategies such has spin caloric thermoelectrics are necessary.

is similar to that of a thermoelectric generator, the energy transfer in spintronic devices mainly takes place by magnons (spin waves) in a ferromagnetic material. These magnons follow Bosonic quasiparticle relation, which permits low energy transmission in the absence of Pauli exclusion requirements. This is a deviation from the classic quasiparticle behavior of Fermionic electrons and holes in p-type and n-type junctions. This provides a possibility to bypass the limitation of conventional charge dynamics.

One possible way to improve the ZT in the conventional thermoelectric modules is by extracting additional voltage from the spin associated with magnons, thereby increasing the power factor, without impacting the fermion transport. This theoretical study employs two fundamental freedoms present in electrons, the charge and the spin, and treats them as independent entities thus paving more freedom to tune the properties and improve the TEs 
performance.

\subsection{Thermoelectric energy conversion}

Solid-state energy conversion devices made of thermoelectric materials are capable of directly converting temperature difference to voltage and are potential candidates for wide range of energy applications.

\subsubsection{Principle of operation of conventional thermoelectrics}

The operation principle of thermoelectric materials can be seen in Figure 1.5, where the temperature gradient across p-type and n-type materials pushes the quasi-particles out of equilibrium causing their respective majority charge carriers (holes and electrons) to move towards the cold surface, thus yielding a voltage difference across their ends sufficient enough to drive electric current through an external load. This was first observed by Thomas Johann Seebeck in the year 1821 when he discovered that a compass needle deflected when it was placed in a closed loop formed by two dissimilar metals, whose ends were maintained at different temperatures. This was due to the creation of current in that closed loop and hence the magnetic field. The EMF generated across the ends is given by Equation 1.4.

$$
E_{e m f}=-S * \nabla T
$$

where $\mathrm{S}$ is Seebeck coefficient, which is a measure of the amount of voltage output per $1 \mathrm{~K}$ temperature bias and $\nabla \mathrm{T}$ is the temperature gradient across the ends. 


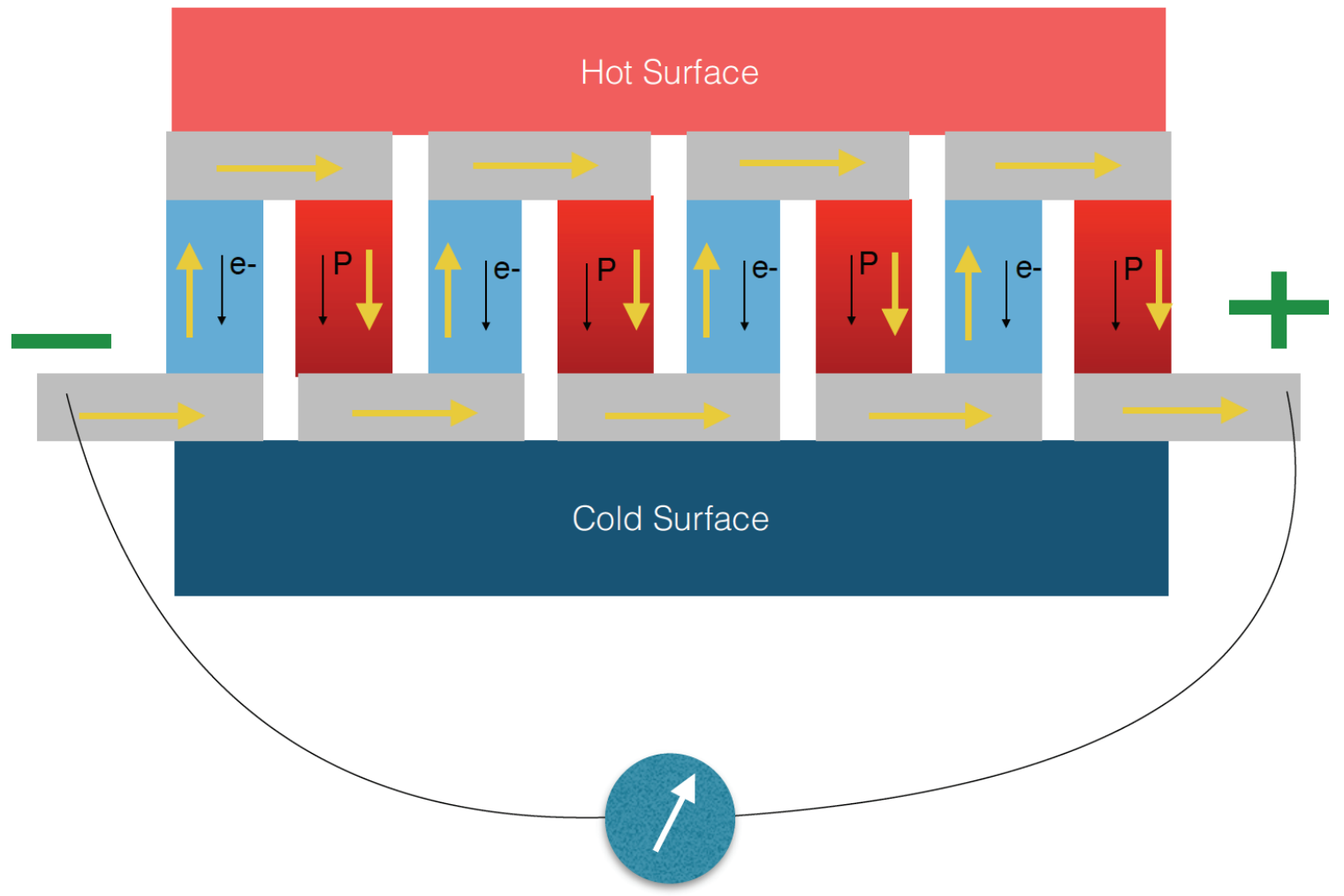

Figure 1.5 Conventional Thermoelectric generator. The series connection of p-type and n-type materials uses the temperature difference across the two surfaces to drive charge carriers.

\subsubsection{Materials used for thermoelectric energy conversion applications}

For the past two decades there has been a surge in thermoelectric research to improve the TE generator performance owing to its potential in waste heat harvesting applications and being silent (no moving parts), reliable and scalable [19]. This surge can also be linked to the advancement in the science of nanostructuring [20] through nano material fabrication technology [3] and quest for green energy over the past decade.

Pure metals are not good thermoelectric materials as they have low Seebeck coefficient and high thermal conductivity. Insulators such as metal oxides have high Seebeck coefficient and low thermal conductivity but the charge density is low, so electrical conductivity is quiet less. Generally, electrical conductivity is accompanied with thermal conductivity. Successful dissociation of these two characteristics can lead to phonon glass electron crystal (PGEC) concept, which means that the material exhibits electronic properties of a crystal and ther- 
mal properties of a glass. Such properties are best desired for any thermoelectric material. Semiconductor materials show substantially better thermoelectric properties compared to metals as a results of the ability to tailor their carrier attributes (ex. majority population).

Substitutional effects have been long tested and proved to successfully enhance the desired properties of materials. In 2006 Y. Nishino et al [21], experimentally tested the influence of $\mathrm{Ge}$ substitution in $\mathrm{Fe}_{2} \mathrm{VAl}$ on lattice thermal conductivity, electrical resistivity and Seebeck coefficient. Substitution of transition metals or heavy metals in general cause reduction of group velocity of phonons along with the increase in phonon scattering probability, which is why it is theorized that heavy metal substitutions increase phonon scattering while keeping the electric conductivity of the material the same. Similar experimental analysis was performed by many research groups who investigated $\mathrm{Pd}$ substitution effect on $\mathrm{NaCo}_{2 x} \mathrm{Pd}_{x} \mathrm{O}_{4}$ [22], elemental substitutions in $\mathrm{Ru}_{2} \mathrm{Ge}_{3}$ [23], $\mathrm{Cu}$ doping in perovskite-type $\mathrm{LaFeO}_{3}$ [24], Nb-doped $\mathrm{Sr}_{0.95} \mathrm{La}_{0.05} \mathrm{TiO}_{3}$ ceramics [25], Cu substituted $\mathrm{Bi}_{2} \mathrm{Ba}_{2} \mathrm{Co}_{2 x} \mathrm{Cu}_{x} \mathrm{O}_{y}$ oxides [26] and heavy element substitution in $\mathrm{Fe}_{2} \mathrm{VAl}$ [27]. It was observed that in each study electrical conductivity and Seebeck coefficient were enhanced while maintaining thermal conductivity to be almost constant. This caused an improvement in the power factor and leading to increase in the zT.

The complexity of the crystal structure also influences the thermal conductivity. With the complexity in the crystal structure of semiconducting oxides the thermal conductivity showed a decreasing trend as determined by Toberer et al [28] and Saniya et al [29]. Different complex structures like Skutterudites [30], Tellurides [31], Perovskites [32], Half-Heuslers [33], and spinels [34] [35] etc, which comprise various metal cations in their material lattices, have a unique interplay between their respective band gap, carrier mobility and effective mass. These were thoroughly investigated by different substitutions thereby engineering the phonon dispersion and band gap of the material. Table 1.1 provides a gist of different material lat- 
tices that were investigated to improve their potential as TE candidates. Each of the complex structures allowed reasonable substitutions whose sole purpose was to decrease thermal conductivity and increase electronic transport to yield higher power factor.

From late 2000's to early 2010's, an approach taken to improve the TE performance was by grain boundary engineering and exploring the inherent lattice structure of layered materials that exhibit better TE properties. Layered thermoelectrics like SnSe [36], [17] showed high ZT value of 2.62 due to its layered structure that exhibits low thermal conductivities and high power factors, making it suitable for TE applications. This inherited property in the structure raised much interest in the field and has potential characteristics of a TE material.

While performance is a necessary quality of a TE material, its cost also plays an important role in selecting the material for specific applications. Table 1.2 provides cost of various material lattices with respect to the crystal structure. It may be noted that the material costs vary from time to time due to demand, availability and international treaties between countries. The table provides a cost information extracted from 2014 cost database prepared by department of energy [56]. The cobalt based spinel oxides were reported to have low cost of $\$ 345 / \mathrm{Kg}$ compared to other materials yet also providing a competitive performance with a ZT 1.4 or an efficiency of around $12 \%$.

Maximising electronic transport while minimizing thermal transport has been the major focus in the TE field. Spin associated with electron has largely been left under-utilised for thermal energy conversion. A new avenue for extracting additional voltage is possible through employing the electrons spin and converting to useful voltage using the phenomena of spin Seebeck effect. The field which studies of the effect of thermal gradient on spin transport in a spin-polarised material is named as spin caloritronics [58]. The past 5 years 
Table 1.1 ZT for different material configurations

\begin{tabular}{|c|c|c|c|}
\hline Material & $\mathrm{ZT}$ & Year Published & Ref. \\
\hline \multicolumn{4}{|l|}{ Skutterudites } \\
\hline $\mathrm{Yb}_{0.19} \mathrm{Co}_{4} \mathrm{Sb}_{12}$ & 1 & 2000 & [37] \\
\hline $\mathrm{In}_{0.25} \mathrm{Co}_{4} \mathrm{Sb}_{12}$ & 1.2 & 2006 & [38] \\
\hline $\mathrm{Ba}_{0.08} \mathrm{La}_{0.05} \mathrm{Yb}_{0.04} \mathrm{Co}_{4} \mathrm{Sb}_{12}$ & 1.7 & 2011 & [39] \\
\hline $\mathrm{Sr}_{0.09} \mathrm{Ba}_{0.11} \mathrm{Yb}_{0.05} \mathrm{Co}_{4} \mathrm{Sb}_{12}+\mathrm{Yb}_{2} \mathrm{O}_{3}$ & 1.6 & 2015 & [40] \\
\hline \multicolumn{4}{|l|}{ Tellurides } \\
\hline $\mathrm{CsBi}_{4} \mathrm{Te}_{6}$ & 0.8 & 2000 & [41] \\
\hline BiSbTe bulk & 1.2 & 2008 & [42] \\
\hline \multicolumn{3}{|l|}{ Perovskites } & {$[31]$} \\
\hline $\mathrm{Sr}_{1-x} \mathrm{La}_{x} \mathrm{TiO}_{3}$ & 0.1 & 2001 & [32] \\
\hline SrTiO3 & 1.7 & 2011 & [39] \\
\hline $\begin{array}{c}\mathrm{CaMn}_{1-x} \mathrm{Nb}_{x} \mathrm{O}_{3} \\
\text { nanocomposites }\end{array}$ & 0.3 & 2008 & {$[43]$} \\
\hline PbTe-Si quantum dots & 0.9 & 1999 & [44] \\
\hline $\mathrm{Pb}_{9.6} \mathrm{~S}_{0.2} \mathrm{Te}_{3} \mathrm{Se}_{7}$ & 1.2 & 2007 & [45] \\
\hline $\mathrm{Si}_{80} \mathrm{Ge}_{20} \mathrm{P}_{2}$ & 1.3 & 2008 & [46] \\
\hline $\mathrm{Bi}_{2} \mathrm{Te}_{2.7} \mathrm{Se}_{0.3}$ & 1.04 & 2011 & [47] \\
\hline $\mathrm{Bi}_{2} \mathrm{Te}_{3}$ & 1.1 & 2012 & [48] \\
\hline \multicolumn{4}{|l|}{ Other Thermoelectric materials } \\
\hline$\beta-\mathrm{Cu}_{2-x} \mathrm{Se}$ & 1.5 & 2008 & [49] \\
\hline $\begin{array}{l}\text { Misfit layered calcium cobaltite } \mathrm{Ca}_{3} \mathrm{Ba}_{0.05} \mathrm{Co}_{4} \mathrm{O}_{9+} \\
\text { Chalcogenides }\end{array}$ & 0.52 & 2015 & {$[50]$} \\
\hline $2 \%$ Na-doped PbTe-PbS & 1.5 & 2011 & [51] \\
\hline $\begin{array}{l}1 \% \text { Al-doped PbSe } \\
\text { Clathrates }\end{array}$ & 1.3 & 2012 & {$[52]$} \\
\hline $\mathrm{Ba}_{8} \mathrm{Au}_{5.3} \mathrm{Ge}_{40.7}$ & 0.9 & 2011 & [53] \\
\hline $\mathrm{Ba}_{8} \mathrm{Ga}_{16} \mathrm{Sn}_{30}$ & 1.45 & 2012 & [54] \\
\hline \multicolumn{4}{|l|}{ layered structures } \\
\hline $\mathrm{SnSe}$ & 2.6 & 2014 & {$[36]$} \\
\hline Na-doped SnSe & 0.8 & 2016 & [55] \\
\hline
\end{tabular}

has seen a surge in this research due to its potential in energy conversion applications and data trasfer in electronics.

The efficiency of thermoelectrics has been limited due to the inherent coupling of the electronic and thermal carriers. Many different approaches like grain boundary scat- 
Table 1.2 Typical Cost per Kg of different material configurations [29] [57]

\begin{tabular}{||ccc||}
\hline Material & Maximum ZT & Average Material Cost (\$/Kg) \\
\hline \hline Cobalt Oxides & 1.4 & $\$ 345$ \\
Clathrates & 1.5 & $\$ 5310$ \\
Chalcogenides & 1.5 & $\$ 730$ \\
Skutterudites & 1.5 & $\$ 562$ \\
\hline
\end{tabular}

tering [59], band structure engineering [60], [61], substitutional effects [62] were incorporated to improve the efficiency of thermoelectrics. Though these methods improved the performance to certain extent, the phonon and electron interactions still hamper the commercial applicability of thermoelectrics. More recently, an avenue to decouple these interactions has been experimental demonstrated using temperature gradient induced spin currents [63], [64], [65], [66], [67], [58]. With this new discovery comes the need for new transport models to understand and optimize their response. In providing a solution, this research is focused on the both the development of a 1D spin-transport model and validating the model using the available experimental data.

\subsubsection{Thermal energy conversion using spin Seebeck effect}

Using spin Seebeck effect, voltage can be generated even from the spin current generated due to heat flowing applied insulating materials. In 2008 Uchida et al [65], experimentally demonstrated the spin current injection from a ferromagnetic film to a non magnetic material due to the application of thermal gradient. Interestingly, this spin current signal can be observed in a macroscopic scale (several millimetres) as opposed to spin diffusion length that can be detected only in few nanometer range. 


\subsubsection{Principle of Seebeck Effect}

When a non-magnetic (NM) metal with high spin orbital coupling (like Pt, Th) is attached on a ferromagnetic material (FM) that is present in an applied thermal bias, a non-equilibrium electron spin polarization in the FM causes a spin current to be injected from the FM into the NM metal. This spin current is then converted into a voltage due to the phenomena of inverse spin Hall effect. This principle is represented in Figure 1.6.

Conventional thermoelectric energy conversion utilizes the principle of Seebeck effect [68] to convert thermal gradient into electric voltage. In this effect, the energy conversion takes place when majority charge carriers drift away from the region of high temperature. A new approach to design thermoelectric modules relies on a slightly different principle involving the electron's spin. The pioneering research in 2008 by Uchida et.al. [65] has opened a new avenue to extract additional heat energy by utilizing the intrinsic angular momentum of electrons, colloquially known as spin. This field that explores charge, spin and energy transport due to temperature difference is called spin caloritronics [64], [69] and is a transpiring field with immense potential in heat conversion applications.

When a thermal bias is applied to any material, the majority charge carriers tend to drift towards the cold side. While in a spin-polarised material, this drift rate is different for spin up and spin down electrons. That particular spin component which has lower effective mass will drift faster towards the cold side compared to the other spin component. This results in redistribution of the spin along the material lattice. Hence, a metal contact attached at different locations on the FM material experiences a different amount of spin injection. This has been experimentally observed by Uchida et al in 2010, where the spin voltage detected by $\mathrm{Pt}$ contacts at different locations along the $\mathrm{LaY}_{2} \mathrm{Fe}_{5} \mathrm{O}_{12}$ magnetic insulator were different as shown in Figure 4.2. The theory behind this effect is discussed more thoroughly in chapter 2 . 


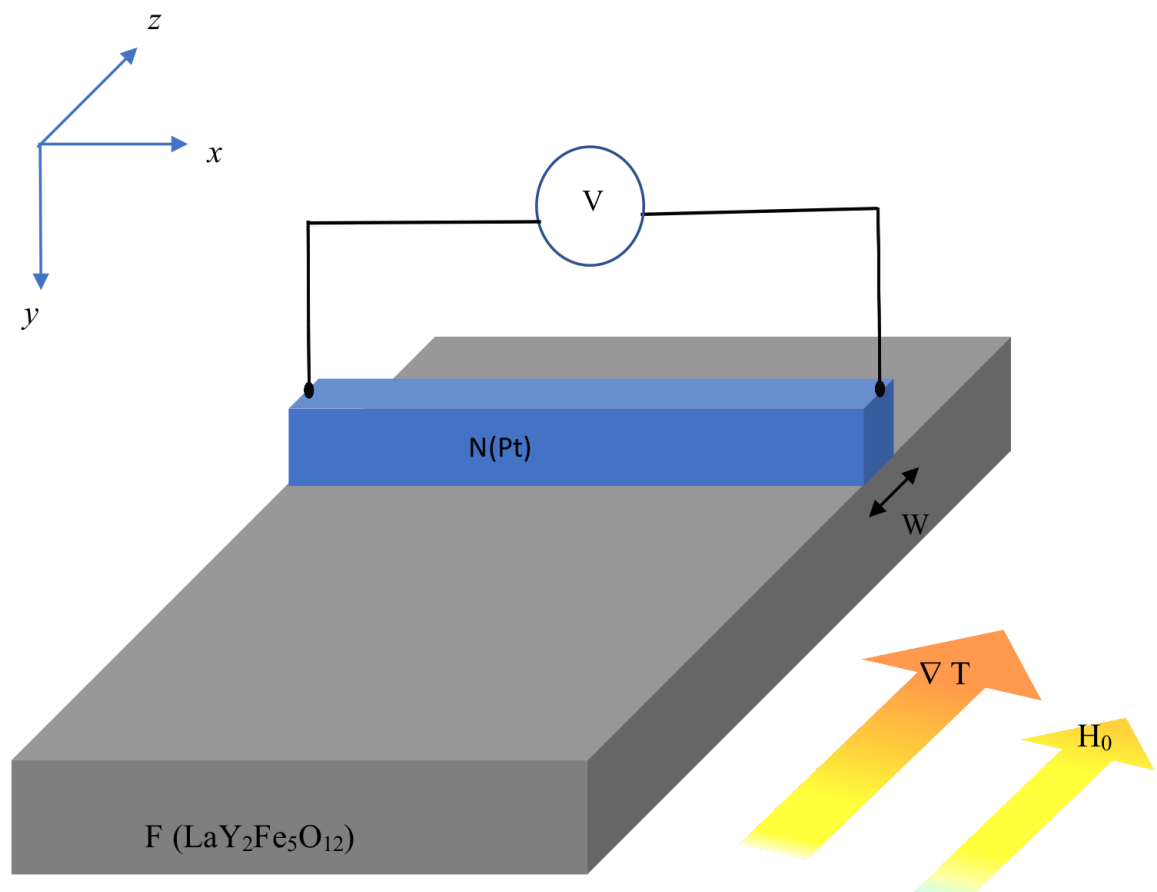

Figure 1.6 Spin Seebeck Effect. A net spin flux enters the metal contact (Pt) when connected on top of a ferromagnetic material that is placed under a net thermal bias. The net spin causes results in a voltage (V) across the two ends of Pt metal contact.

There are two types of configurations that are capable of converting the spin current to voltage. A longitudinal configuration, shown in 1.8 where the $\nabla \mathrm{T}$ and $\mathrm{J}_{s}$ are parallel to each another while a transverse configuration where $\nabla \mathrm{T}$ and $\mathrm{J}_{s}$ are perpendicular as shown in Figure 1.8. In longitudinal configuration, entire charge current is converted to spin current and converted to voltage in the attached NM metal, while in a transverse configuration the NM contacts experience a different spin current at different locations along the material. Longitudinal configuration employs only spin component of electrons to generate voltage while the transverse configuration accomodates a facility to extract both spin and charge current to generate voltage. Hence in addition to the charge current, an additional spin 


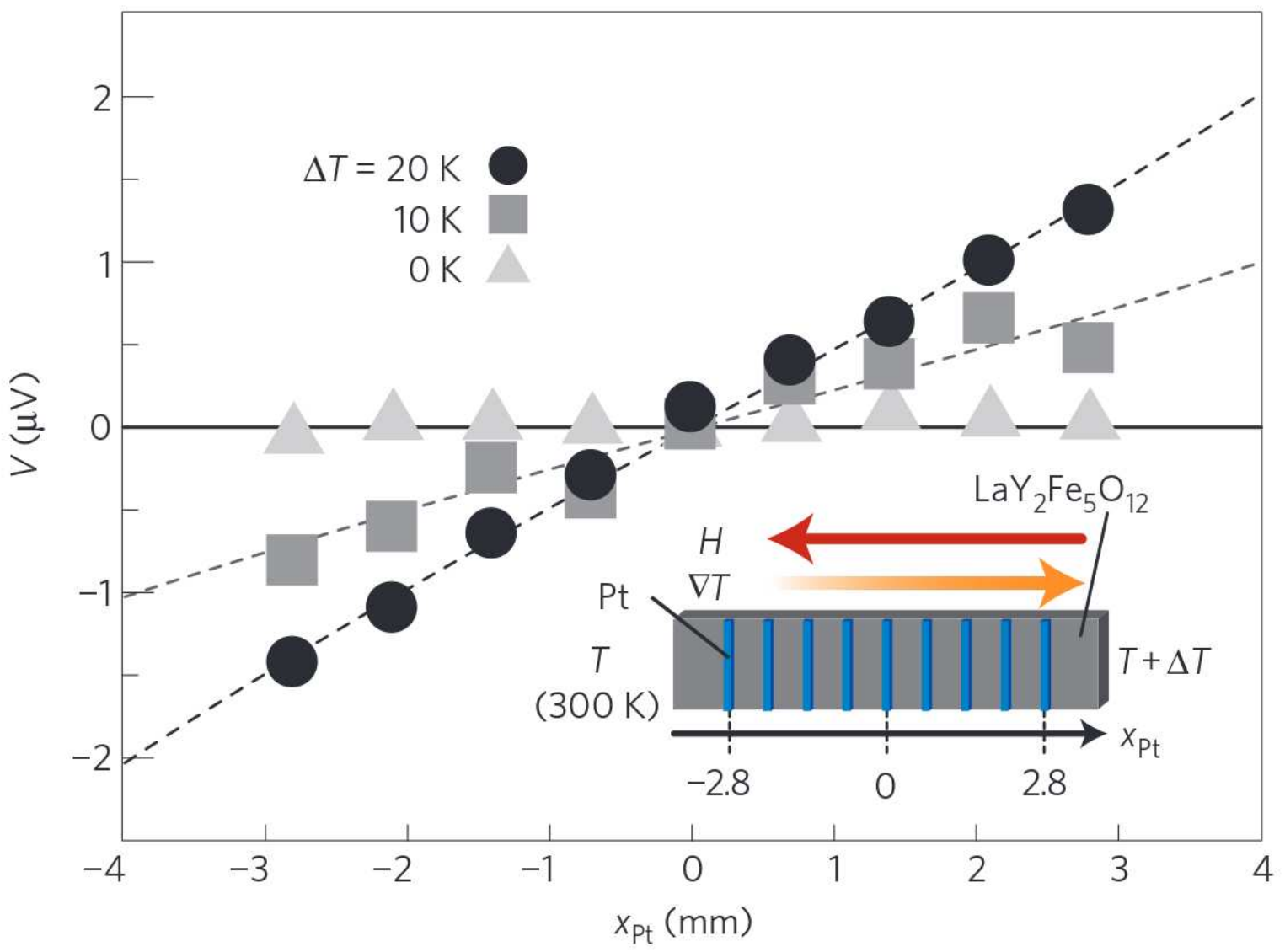

Figure 1.7 Spin voltage induced into Pt contacts at various locations along the La:YIG device [67]. There is a linear dependence on the voltage and position along the device.

current can be extracted in a transverse configuration.

\subsection{Composite Spin and Conventional Thermoelectric Generator}

The combined conventional and spin based thermoelectic design is shown in Figure 1.9. Here both n-type and p-type magnetic materials have Pt contact electrodes deposited transversely near the cold side. There are two interconnect frameworks, one in blue the other in red, throughout the TE module that collect the generated spin voltage. As each side of the TE material is capable of injecting spin into the Pt contact, each of the square cross-sectioned pillar equips four Pt contacts that convert spin current to electric voltage, hence in theory, there can be a 4 fold increase in the spin voltage. 


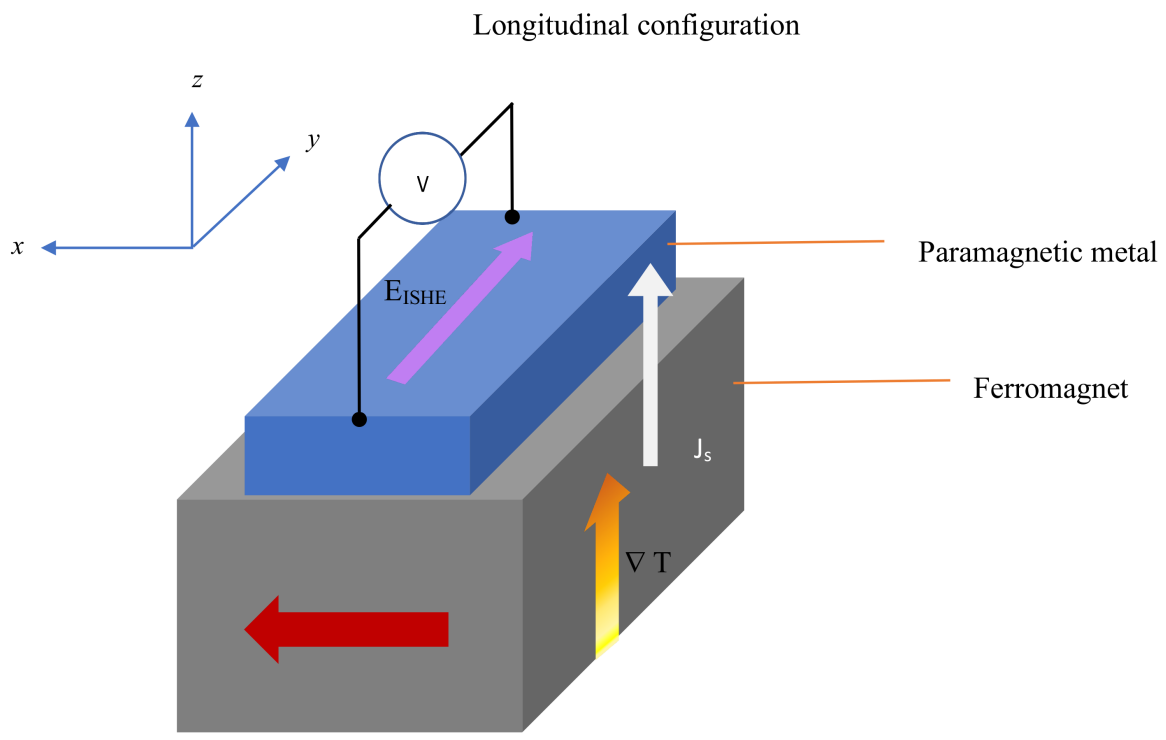

Transverse configuration

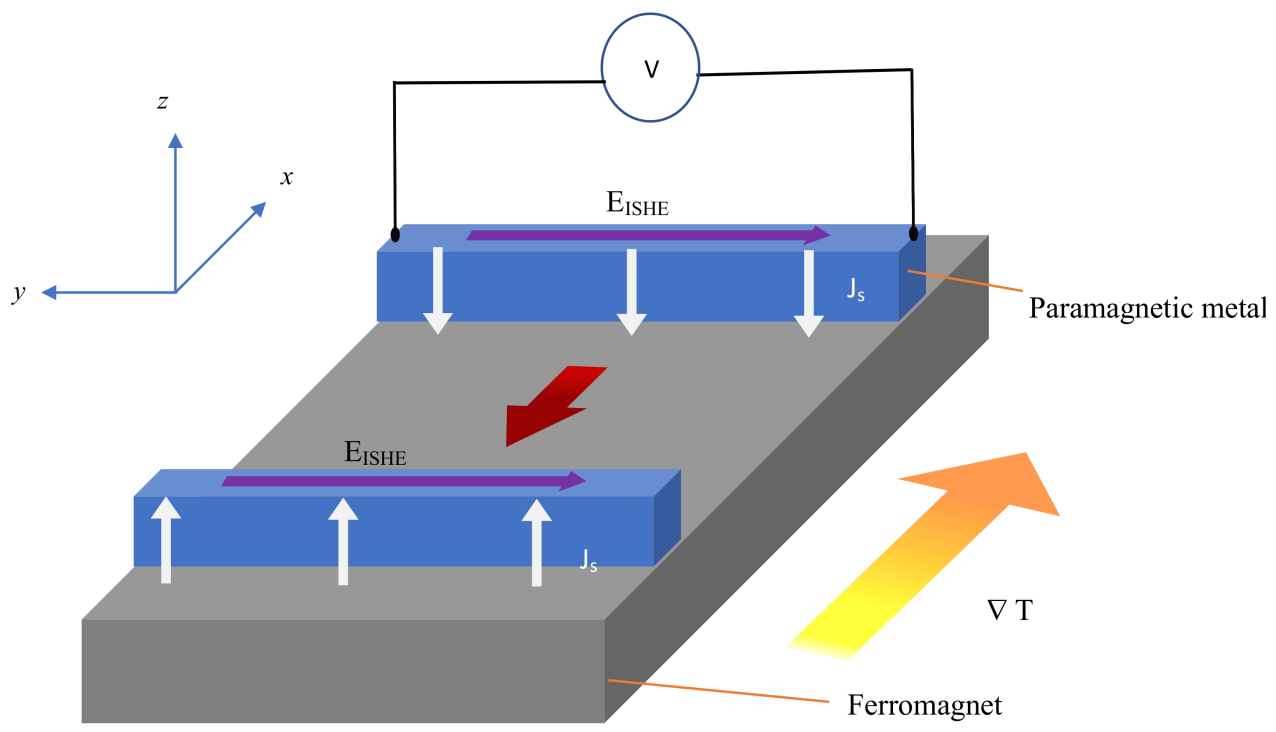

Figure 1.8 Top figure is a longitudinal configuration where the spin contact is perpendicular to the magnetic polarization. The bottom figure is a transverse configuration (configuration studied within) and is configured when a Pt metal contact is placed on the ferromagnet such that spin current is perpendicular to the heat gradient. 
In the Figure 1.9, the enlarged view of the p-type and n-type leg is shown. overlap of the frameworks is depicted with an arc that connects positive and negative terminals. To eliminate the possibility of interconnect overlap, entire blue framework can be deposited below the red framework and can extract voltage. So, in addition to charge voltage that is in effect in current conventional thermoelectrics, a spin voltage can also be extracted from spin-polarized magnetic materials, which when combined can yield a higher voltage thereby increasing the ZT of the material.

Compared to clathrates or half-heuslers alloys, silicides or tellurides, Co based spinel oxides are relatively cheaper and also abundantly available [70]. This research investigates a low cost, spin-polarized and complex structured cobalt spinels due to their potential in spin caloritronic applications.

\subsection{Cobalt containing spinel oxides}

To extract the spin contribution towards generation of additional voltage, the material has to be spin polarized. Spinel material lattice has one such feature of accommodating cations with different oxidation states. Wide range of transition metals can be substituted in various sites inside the lattice that contribute to a net spin-polarization. The compounds exhibiting spinel structure are represented by a generic formula $\mathrm{AB}_{2} \mathrm{O}_{4}$ where $\mathrm{A}$ and $\mathrm{B}$ are metal cations occupying (8) tetrahedral and (16) octahedral sites with an oxidation state of +2 and +3 , respectively in a 56 atom unit cell. Figure1.10 shows a spinel structure arrangement of atoms in a cubic unit cell. In the case of $\mathrm{AB}_{2} \mathrm{O}_{4}$ exhibiting a normal spinel structure, all $\mathrm{A}^{+2}$ cations occupy tetrahedral sites (coordination number 4 ) and $\mathrm{B}^{+3}$ cations occupy octahedral sites (coordination number 6). Whereas in the case of inverse spinel structure all $\mathrm{A}^{+2}$ cations occupy half of octahedral sites and $\mathrm{B}^{+3}$ cations fill the remaining octahedral and tetrahedral sites. 

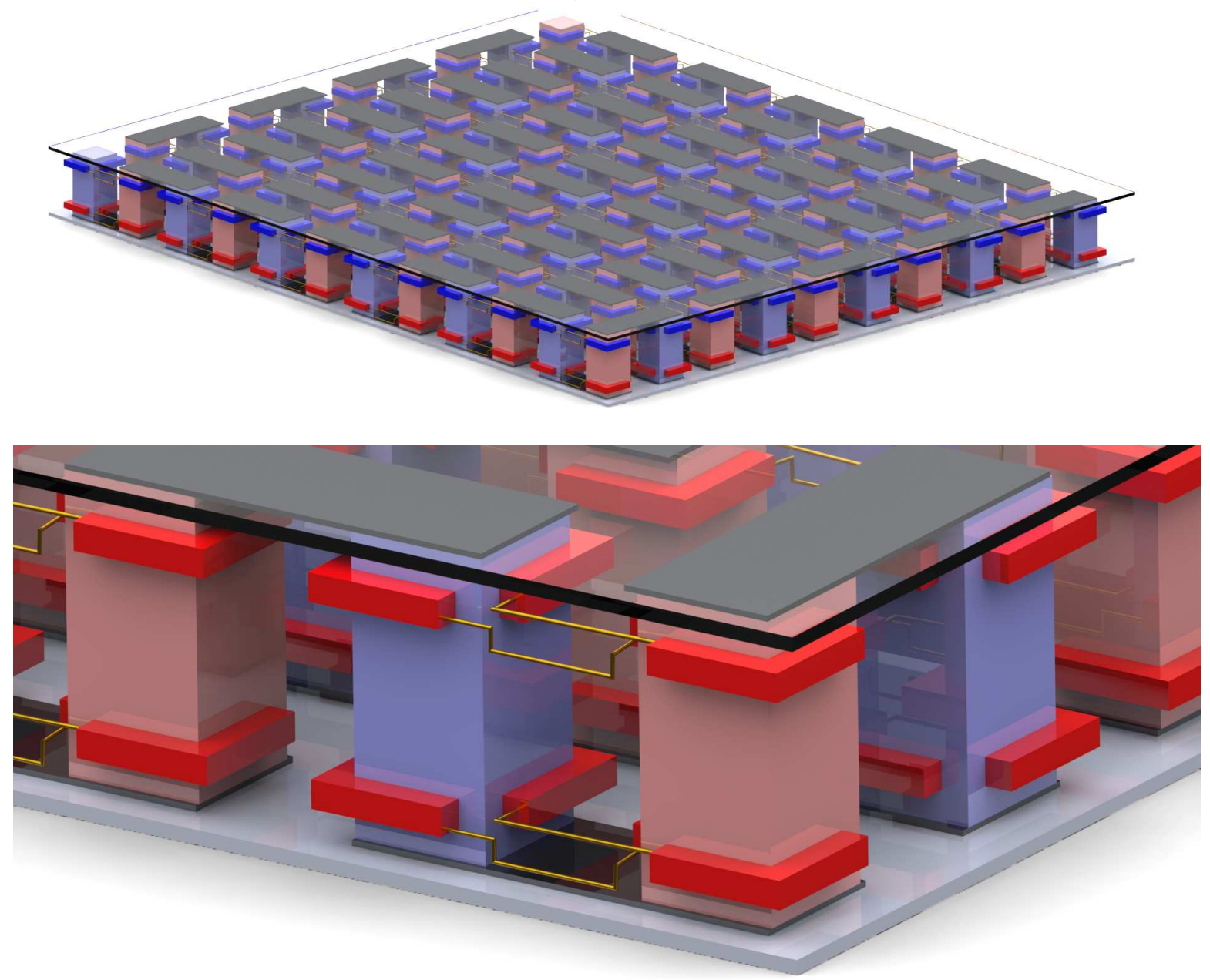

Figure 1.9 Top is an isometric view of the combined conventional and spin based thermoelectric material. Bottom is an enlarged view of the thermoelectric generator showing the Pt metal contacts (in red color) attached on the transverse direction to the p-type and n-type thermoelectric legs. These Pt contacts generate additional spin voltage which can be combined with the conventional charge voltage to result in an increase of the overall ZT of the material.

A study of series of solid solutions of $\mathrm{Co}_{3-x} \mathrm{Ni}_{x} \mathrm{O}_{4}$ [71] and $\mathrm{Co}_{3-x} \mathrm{ZnO}_{4}[72], 0 \leq \mathrm{x} \leq 1$, by Appandairajan et al; showed more than 10 folds increase in electrical conductivity while the Seebeck coefficient decreased. As discussed in the previous section, for optimal performance of any thermoelectric material, high power factor with lower thermal conductivity is 


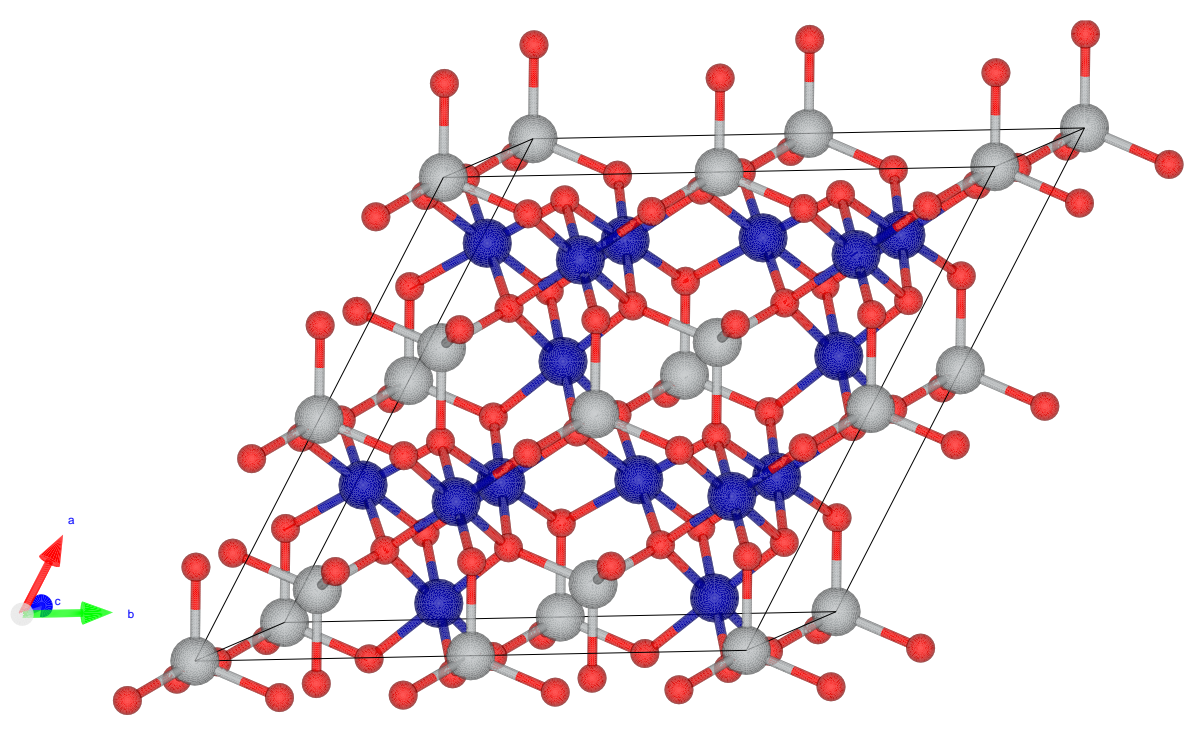

Figure 1.10 A super cell of a 2x2x1 primitative unit cells that is based on a spinel structure comprising 56 atoms. The silver balls represent tetrahedral sites which have coordination number 4 , the blue balls represent octahedral sites which have coordination number 6 and the red balls represent oxygen atoms. The difference in spin between the tetrahedral and octahedral sub lattice allows control of the ferrimagnetic properties of the structure.

desired. It is estimated that by substituting Co with $\mathrm{Zn}$ and $\mathrm{Ni}$, the thermal conductivity can be decreased while improving electrical conductivity. As represented in Figure1.11 an intermediate configuration comprising right amount of $\mathrm{Zn}$ and $\mathrm{Ni}$, the configuration of which can be written as $\mathrm{Co}_{3-x-y} \mathrm{Zn}_{x} \mathrm{Ni}_{y} \mathrm{O}_{4}$ for $0 \leq \mathrm{x}+\mathrm{y} \leq 1$, is expected to show enhanced thermoelectric properties compared to pure $\mathrm{Co}_{3} \mathrm{O}_{4}$.

In addition to the promising behaviour as conventional thermoelectrics, the normal spinel configuration, $\left(\mathrm{Co}^{+2}\right)_{\text {tet }}\left(\mathrm{Ni}_{x}^{+3} \mathrm{Co}_{2-x}^{+3}\right)_{\text {oct }} \mathrm{O}_{4}$, and inverse spinel configurations $\left(\mathrm{Co}^{+3}\right)_{\text {tet }}\left(\mathrm{Ni}_{x}^{+2} \mathrm{Co}_{2-x}^{+3}\right)_{\text {oct }} \mathrm{O}_{4}$ have high net spin polarization in them. This enables these materials to be capable of generating spin current in the presence of heat gradient. Hence, Ni and Zn substituted cobalt spinel oxides are studied in this research for their spin thermoelectric capability.

Cobalt based spinels have attracted wide applications as electrodes in $\mathrm{H}_{2} \mathrm{O}$ electrolysis [73], p-type carrier-selective interlayers in organic solar cells [74], supercapacitors, transpar- 


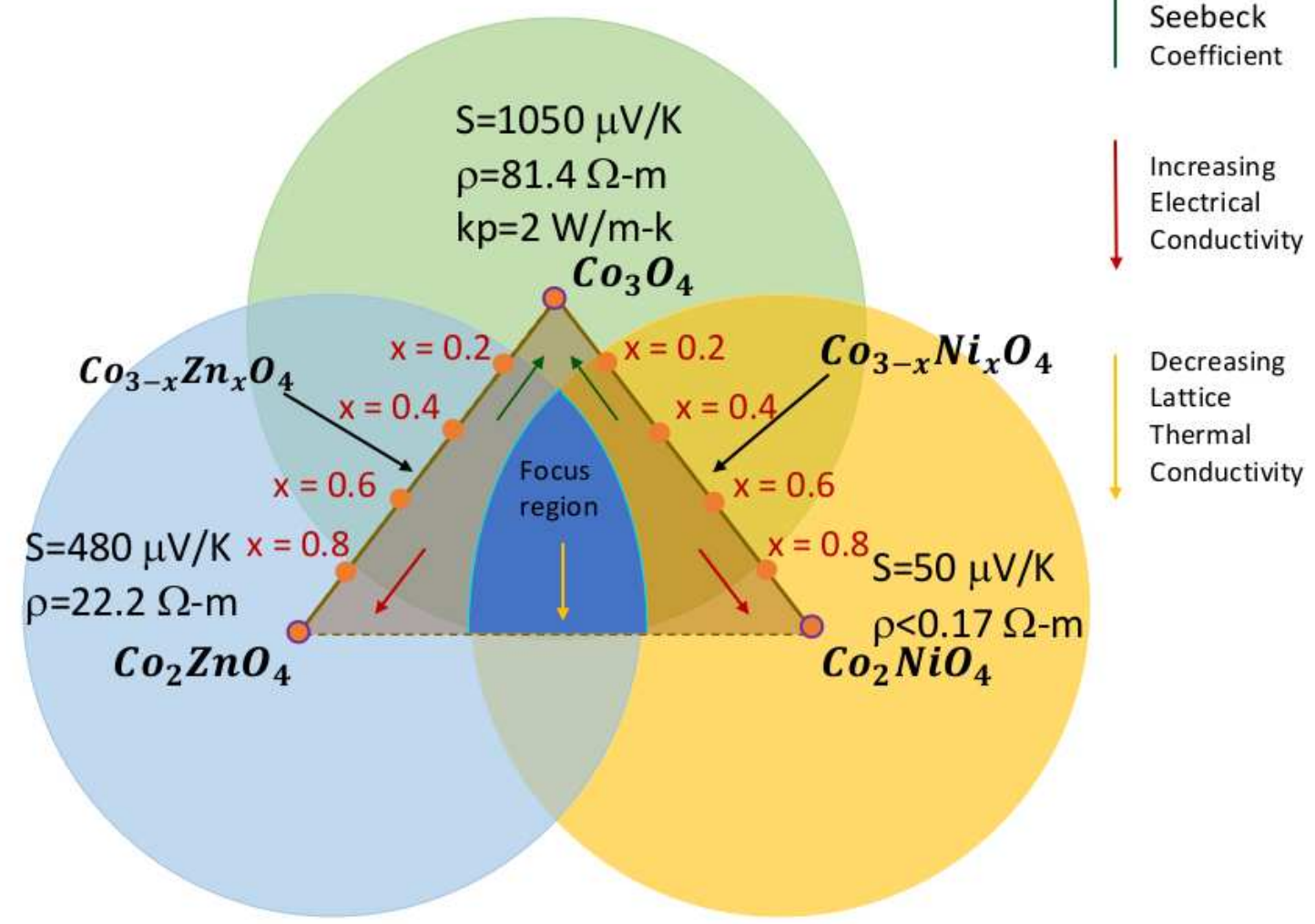

Figure 1.11 Compositional space comprising $\mathrm{Co}_{3} \mathrm{O}_{4}, \mathrm{Co}_{2} \mathrm{ZnO}_{4}$ and $\mathrm{Co}_{2} \mathrm{NiO}_{4}$ as end points. ent conductor films [75], gas sensors [76], CO oxidizing materials [77]. There have been experimental investigations on $\mathrm{Co}_{3-x} \mathrm{Ni}_{x} \mathrm{O}_{4}$ like the work by $\mathrm{N}$. Bahlawane et al [78], M. Guene et al [79] and N.K. Appandairajan et al [71] that demonstrated a large increase in electrical conductivity with Ni substitution in the spinel matrix of cobalt, similarly the transition from antiferromagnetic to ferrimagnetic behavior with the $\mathrm{Ni}$ has been verified by N.K.Appandairajan et al [80]. Though the scope of this compound for various applications is high, a fundamental understanding of the doping influence is not well understood. By understanding the mechanism for this large increase in conductivity the properties can be tailored for a particular application. Therefore, this research investigates the influence of nickel and zinc on the properties of cobalt containing spinels which can assist in predicting the ground state properties of other intermediate compositions. 
When $\mathrm{Ni}$, which is stable in +2 or +3 oxidation state in a spinel structure, is added to this compound, it preferentially substitutes $\mathrm{Co}^{+3}$ in octahedral sites [81] resulting in an inverse spinel structure. Though the preferential substitution of $\mathrm{Ni}^{+2}$ in octahedral sites has appeared ambiguous, the relative tendency for $\mathrm{Ni}$ to form high spin configuration in a spinel structure can be taken into account when studying the ground state properties. Hence, with $\mathrm{Ni}^{+2}$ in tetrahedral sites leading to a normal spinel structure and $\mathrm{Ni}^{+2}$ in octahedral sites leading to an inverse spinel structure or a partial inverse spinel structure, that is caused due to the presence of $\mathrm{Ni}^{+2}$ in both tetrahedral and octahedral sites, can be studied for their respective ground state properties and to create an insight over 10 fold increase in its electrical conductivity due to tetragonal distortion [78].

In the case of Zn substitution into the spinel lattice, the initial study can be performed with $\mathrm{Zn}^{+2}$ substituting $\mathrm{Co}^{+2}$ in tetrahedral sites forming a normal spinel. It has been experimentally verified that $\mathrm{Zn}^{+2}$ preferentially substitutes $\mathrm{Fe}^{+2}$ in $\mathrm{Fe}_{3-x} \mathrm{Zn}_{x} \mathrm{O}_{4}$ spinel configuration [78], [82]. Also, the experimental work by N.H. Perry et al; confirms that a solid solution exists for $\mathrm{Co}_{3} \mathrm{O}_{4}-\mathrm{Co}_{3-x} \mathrm{ZnO}_{4}$ fro $0 \leq \mathrm{x} \leq 0.9$ [83]. Now, with the $\mathrm{Zn}$ and $\mathrm{Ni}$ substitutions in Co spinel lattice, the electrical conductivity showed an upward trend, while for both cases Seebeck coefficient has decreased considerably. A first principle investigation of the solid solution lines for $\mathrm{Co}_{3} \mathrm{O}_{4}-\mathrm{Co}_{2} \mathrm{ZnO}_{4}$ and $\mathrm{Co}_{3} \mathrm{O}_{4}-\mathrm{Co}_{2} \mathrm{NiO}_{4}$ can elucidate the reason behind the enhancement in electrical conductivity which leads to a configurational space of further interest.

The approach taken in this research was to use density functional theory (DFT) to investigate $\left(\mathrm{Co}_{1-x}^{+2} \mathrm{Co}_{x}^{+3}\right)_{\text {tet }}\left(\mathrm{Ni}_{x}^{+2} \mathrm{Co}_{2-x}^{+3}\right)_{\text {oct }} \mathrm{O}_{4}$ and $\left(\mathrm{Zn}_{x}^{+2} \mathrm{Co}_{1-x}^{+2}\right)_{t e t}\left(\mathrm{Co}_{2}^{+3}\right)_{\text {oct }} \mathrm{O}_{4}$ for $\mathrm{x}$ ranging from 0 to 1 to calculate the bonding, electronic and magnetic properties. Furthermore, the electrical conductivity as a function of oxidation state of $\mathrm{Ni}$ in octahedral coordination and the 
evolution from spinel to inverse spinel can be investigated.

The normal and inverse spinel configurations can be represented by $\left(\mathrm{Co}^{+2}\right)_{t e t}\left(\mathrm{Ni}_{x}^{+3} \mathrm{Co}_{2-x}^{+3}\right)_{\text {oct }} \mathrm{O}_{4}$ and $\left(\mathrm{Co}^{+3}\right)_{t e t}\left(\mathrm{Ni}_{x}^{+2} \mathrm{Co}_{2-x}^{+3}\right)_{\text {oct }} \mathrm{O}_{4}$ respectively. Here, $\mathrm{Ni}^{+3}$ and $\mathrm{Ni}^{+2}$ represent low spin and high spin configurations respectively, whose crystal field splitting according to crystal field theory is shown in Figure1.12. Hence, to study the effect of increasing concentration of $\mathrm{Ni}$ in Octahedral sites, ground state properties will be calculated for normal and inverse spinel configurations of $\left(\mathrm{Co}^{+2}\right)_{t e t}\left(\mathrm{Ni}_{x} \mathrm{Co}_{2-x}^{+3}\right)_{\text {oct }} \mathrm{O}_{4}$ for each $\mathrm{x}=0.0,0.125,0.25,0.375,0.5,0.625$, 0.750, 0.875 and 1.0 using DFT $+\mathrm{U}$ approach on a $2 \times 2 \times 1$ supercell.

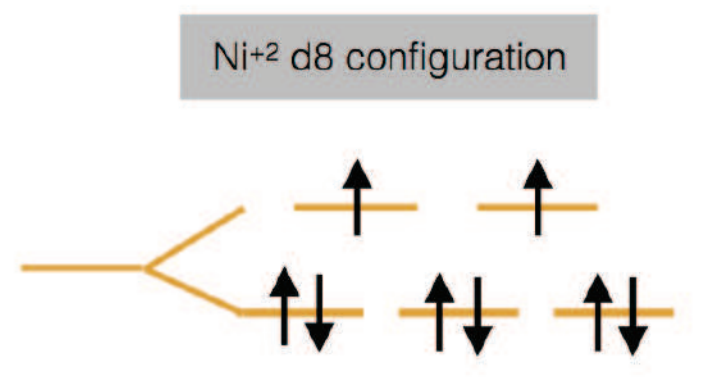

$\mathrm{Ni}^{+3} \mathrm{~d} 7$ configuration

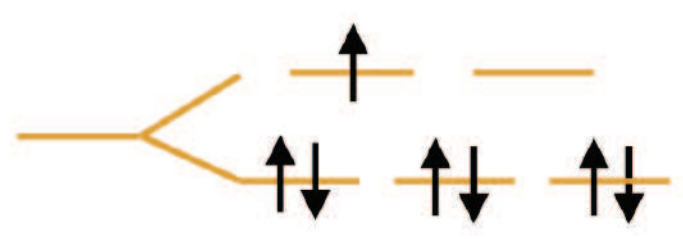

\section{$\mathrm{Co}^{+2} \mathrm{~d} 7$ configuration}

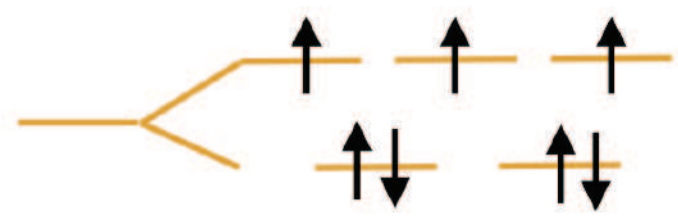

$\mathrm{Co}^{+3} \mathrm{~d} 6$ configuration

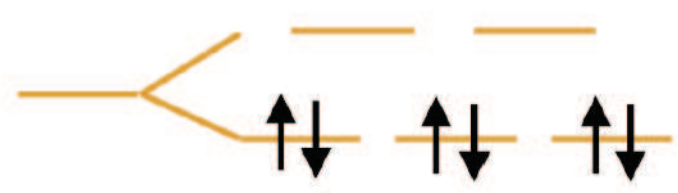

Figure 1.12 Crystal field splitting of $\mathrm{Ni}^{+2}, \mathrm{Ni}^{+3}, \mathrm{Co}^{+2}$ and $\mathrm{Co}^{+3}$ configurations

Also, to study the effect of increasing concentration of $\mathrm{Zn}$ in tetrahedral sites, ground state properties will be calculated for normal spinel configurations of $\left(\mathrm{Zn}_{x}^{+2} \mathrm{Co}_{1-x}^{+2}\right)_{t e t}\left(\mathrm{Co}_{2}^{+3}\right)_{\text {oct }} \mathrm{O}_{4}$ for each $\mathrm{x}=0.0,0.125,0.25,0.375,0.5,0.625,0.750,0.875$ and 1.0 using DFT $+\mathrm{U}$ approach on a $2 \mathrm{x} 2 \mathrm{x} 1$ supercell. 
In order to theoretically predict the inverse spin Hall voltage $\left(\mathrm{V}_{I S H E}\right)$ of spin polarized materials, we developed a 1-D spin transport model by combining non equilibrium Green's function formalism (NEGF) [84] and spin transport theory [85]. In this approach, first the fundamental parameters of a material such as lattice constant, band gap, Fermi energy, effective mass and magnetization of the material lattice, were calculated using density functional theory (DFT). The NEGF model used this information to calculate the surface current for the spin channels independently and spin transport theory will then be used to calculate the spin current injection and inverse spin Hall voltage generated in the attached metal contact.

NEGF modeling has been implemented in various studies to describe the quantum transport in different materials in the presence of thermal bias [86]' [87]. There also available softwares like TranSIESTA [88] that incorporate the application of this formalism. A combination of DFT and NEGF [86]; or NEGF and spin transport theory [87], were used to describe the electron transport but a combination of DFT+NEGF+spin transport theory to calculate the $\mathrm{V}_{I S H E}$ in magnetic materials is near to less in literature. Hence, this research article aims to develop a 1-D model using a combination of NEGF formalism and spin transport theory by incorporating the parameters obtained from DFT calculations.

Transverse spin Seebeck configuration, Figure 1.8, which is the major scope of this research, has been experimentally verified using La:YIG [67] and NiFe [89]. Insulating magnetic materials like La:YIG displaying spin Seebeck effect is a strong indication of magnaon driven spin Seebeck that is caused due to spin redistribution in a material and also proves the capability of spin carriers in a material lattice to generate voltage. In addition to this, semiconducting or insulating oxide magnetic materials have immense scope in this emerging field due to their ability to accommodate wide variety of substitutions and tune various electronic and magnetic properties. Hence we chose La:YIG as our material of study to validate and verify the developed model. $\mathrm{LaY}_{2} \mathrm{Fe}_{5} \mathrm{O}_{12}$ (La:YIG) material lattice is obtained by substituting one Yttrium with Lanthanum in $\mathrm{Y}_{3} \mathrm{Fe}_{5} \mathrm{O}_{12}$ (YIG). There have been few theoretical 
studies reporting the fundamental property study using DFT to calculate lattice parameter, electronic band structure and effective mass data for YIG [90], [91], [92] but there is no available electronic band structure data for La:YIG. Hence, first principle calculations based of DFT were performed on YIG to compare the effective mass and band gap data with literature available for YIG, and a similar approach has been applied to calculate the electronic band structure data for La:YIG. 


\section{CHAPTER 2}

\section{COMPUTATIONAL APPROACH AND MODEL DEVELOPMENT}

\subsection{Density Functional Theory}

First principle density function theory (DFT) is a quantum mechanical modeling tool useful to predict ground state electronic structure. DFT's ability to reduce the interaction between 1 mole of electrons ( $10^{23}$ ) using an approximate Schrödinger equation to a single particle effective theory, makes it widely applicable to approximate electronic, magnetic, spectroscopic and structural properties of materials. The calculation of fundamental properties is done by solving the non-relativistic Kohn-Sham equations [93] that incorporate DFT formalism which is mainly based on Hohenberg-Kohn theorem [94]. Hohenberg-kohn theorem states that,

1. All ground state properties of a system, including total energy, are some functional of the ground state charge density,

2. The correct ground state charge density minimizes the functional.

According to Kohn-Sham, the total energy of the system is given by,

$$
E(n)=T_{s}(n)+E_{H}(n)+E_{x c}(n)+\int V_{e x t}(r) n(r) d r
$$

where $\mathrm{n}$ is the charge density, $\mathrm{T}_{s}(\mathrm{n})$ is the kinetic energy of the non-interacting system, $\mathrm{E}_{H}(\mathrm{n})$ is the Hartree energy, $\mathrm{E}_{x c}(\mathrm{n})$ is the exchange-correlation energy functional which contains information pertaining to exchange interactions between electrons and all other correlational effects in a many-electron system and $\mathrm{V}_{\text {ext }}(\mathrm{r})$ is the exchange-correlation potential. The exchange-correlation energy functional, $\mathrm{E}_{x c}(\mathrm{n})$, can be approximated in DFT 
by LSDA (local spin density approximation) or GGA (generalised gradient approximation). In this thesis, DFT calculations were performed through plane-wave basis incorporated in Quantum Espresso package [95].

By varying the charge density until the global minimum in the energy functional is reached, ground state of that system is achieved. In order to solve the Equation 2.1, exchange-correlation functional approximated by a commonly known approximation method called Local-density approximation (LDA), in which exchange-correlation energy only depends on the density is evaluated.

$$
E_{x c}^{L D A}(n)=\int n(r) \epsilon(n(r)) d r
$$

In Equation $2.2 \epsilon(n(r))$ is the exchange correlation energy per particle with electron density $n(r)$. In an attempt to improve LDA, generalized gradient approximation (GGA) is developed where it assumes a gradient of the electron density at any point in space. In this work, the improved approximation method, GGA, was used to approximate the functional.

For the complete calculation of the thermoelectric properties the approach taken was to derive several material attributes from the ground state calculation. These parameters were then used in the transport model, which will be discussed in the next section. The parameter derived from the DFT calculation include the effective mass $\left(\mathrm{m}^{*}\right)$, Fermi energy, band edges, magnetization, and lattice parameter. All these parameters were obtained from band structure, structural and magnetization data from a fully relaxed (low energy state) supercell. This was carried out for a range of composition and sublattice configurations. Perdew, Burke and Ernzerhof (GGA-PBE) [96, 97] approximation was used to describe the exchange-correlation functional as implemented in the Quantum-Espresso package [95]. Ultrasoft pseudopotentials were used to describe the interaction between the ions and electrons. 
The selection of GGA-PBE is based on its potential in correcting for over-binding in strongly correlated condensed matter that arises from the over analyticity of the funcationals. The pseudopotentials were provided from the PSLibrary 1.0.0 database.

$\mathrm{Ab}$ initio calculations of the electronic band structure and structural properties were performed based on density functional theory using the plane wave scheme as implemented in Quantum Espresso package [95]. In all the calculations of YIG and La:YIG materials, the plane wave energy cut-off of $1220 \mathrm{eV}$ was used to yield high convergence in energies, where the relative energy convergence of $10^{-9} \mathrm{eV}$ was implemented. The structures were relaxed until the forces on each ion reached below $1 \mathrm{meVA}^{-1}$. All the calculations were spinpolarized and the atomic cores were described by ultrasoft pseudo potentials [98], as they efficiently handle localised electrons and provide accurate results comparable to all-electron calculations. The atomic valance of $4 \mathrm{~s}^{1} 3 \mathrm{~d}^{7}, 5 \mathrm{~s}^{2} 4 \mathrm{~d}^{1}$ and $2 \mathrm{~s}^{2} 2 \mathrm{p}^{4}$ was used for Fe, $\mathrm{Y}$ and $\mathrm{O}$ in the respective pseudopotentials for YIG and La:YIG materials. Calculations were performed on 80 atom super cell using a 4x4x4 Monkhorst-Pack k-point grid.

\subsection{Pseudopotential Generation}

Pseudopotential is an approximation where an effective potential is generated to describe an atom assuming core electrons to be frozen, as the valance electrons surrounding the core region are the ones that effectively contribute in bonding properties. The electrons present at the core of an atom are highly localized and are tightly bound to the nucleus and moreover the energy of such electron core falls way below the conduction band and hence are assumed to be frozen. The valance electrons described by the pseudo-wavefunctions with fewer nodes significantly contributes in making the plane-wave basis set very practical to use. Hence this effective potential when used reduces the computational time. 
For Co atoms and $\mathrm{Ni}$ atoms, the respective electrons in $3 \mathrm{~d}^{7} 4 \mathrm{~s}^{2}$ and $3 \mathrm{~d}^{8} 4 \mathrm{~s}^{2}$ were treated to be the valance electrons and the remaining core shell electrons were replaced by their respective core potentials. The calculations were performed with a planewave kinetic energy cut off of $1225 \mathrm{eV}$ on a 56 atom supercell with 8x8x8 k-point grid. All the calculations were spin polarized. The Hubbard U was calculated by performing a series of calculations for different values ranging from 2 to $6 \mathrm{eV}$ for each of $\mathrm{Co}$ and $\mathrm{Ni} 3 \mathrm{~d}$ orbitals. After comparing the resulting band gap and magnetic moment to the literature, the values of 4.4 and $3.2 \mathrm{eV}$ for Co and Ni respectively, were chosen to maintain consistency with the results. For each composition, the structures were relaxed until the forces on each ion reached below $1 \mathrm{meVA}^{-1}$.

\subsection{Transport Model Theory}

Using DFT, a supercell arrangement or a system of isolated molecules can be solved for their electronic structure relaxation type solid state physics problems. In case of quasiparticle transport phenomena calculations across two boundaries, the effects of chemical potential due an external bias or variation of charge density in the scattering region while considering temperature effects cannot be solved using DFT alone to replicate the boundary conditions in a quantum transport. However, the combination of DFT with NEGF formalism is a powerful tool to study quantum transport phenomena in nanoscale region. The NEGF formalism is a self-consistent method, where Schrödinger's equation and Poisson's equation are solved self-consistently for a copmosite effective mass system.

\subsubsection{Non-Equilibrium Green's Function (NEGF) formalism}

A composite system modeled in this research is shown in Figure 2.1 which is divided into four regions, i.e., the left contact, the scattering region, the right contact and the in- 
verse spin Hall effect electrode (ISHE) which typically is Pt metal. The ISHE electrode is a conceptual floating probe used to calculate net spin flux flow between the FM and NM at various locations along the lattice. These probes are conceptually used to extract electrons from the device or inject into the device, at the region of study, to effectively calculate the scattering and transmission of electrons due to the applied temperature bias. The model incorporates the scattering effects that include connection of the channel to the contacts on the two ends and interactions withing the channel. To describe the system, two components that represent outflow $\left[\Sigma^{\text {out }}\right]\{\psi\}$ and inflow $\{\mathrm{s}\}$ from the contacts should be added to the usual time-independent Schrödinger equation represented by $\mathrm{E}\{\psi\}=[\mathrm{H}]\{\psi\}$ which is given by the Equation 2.3 .

$$
E\{\psi\}=[H]\{\psi\}+\left[\Sigma^{o u t}\right]\{\psi\}+\{s\}
$$

where $\psi$ is the many-particle wave function between the contacts.

From the above, $\{\psi\}$ can be written as the following,

$$
\{\psi\}=[G]\{s\} .
$$

Where $[\mathrm{G}]$ is,

$$
\begin{array}{r}
{[G]=\left[E I-H-\Sigma^{\text {out }}\right]^{-1},} \\
\text { replacing } \quad \Sigma^{\text {out }}=\Sigma_{l}^{\text {out }}-\Sigma_{r}^{\text {out }}, \\
{[G]=\left[E I-H-\Sigma_{l}^{\text {out }}-\Sigma_{r}^{\text {out }}\right]^{-1} .}
\end{array}
$$

Here, the $\Sigma_{l}^{\text {out }}$ and $\Sigma_{r}^{\text {out }}$ terms are the outflow energies at the left and right contacts respectively. In addition to the outflow and inflow terms, the electrostatic potential energy (U) of 


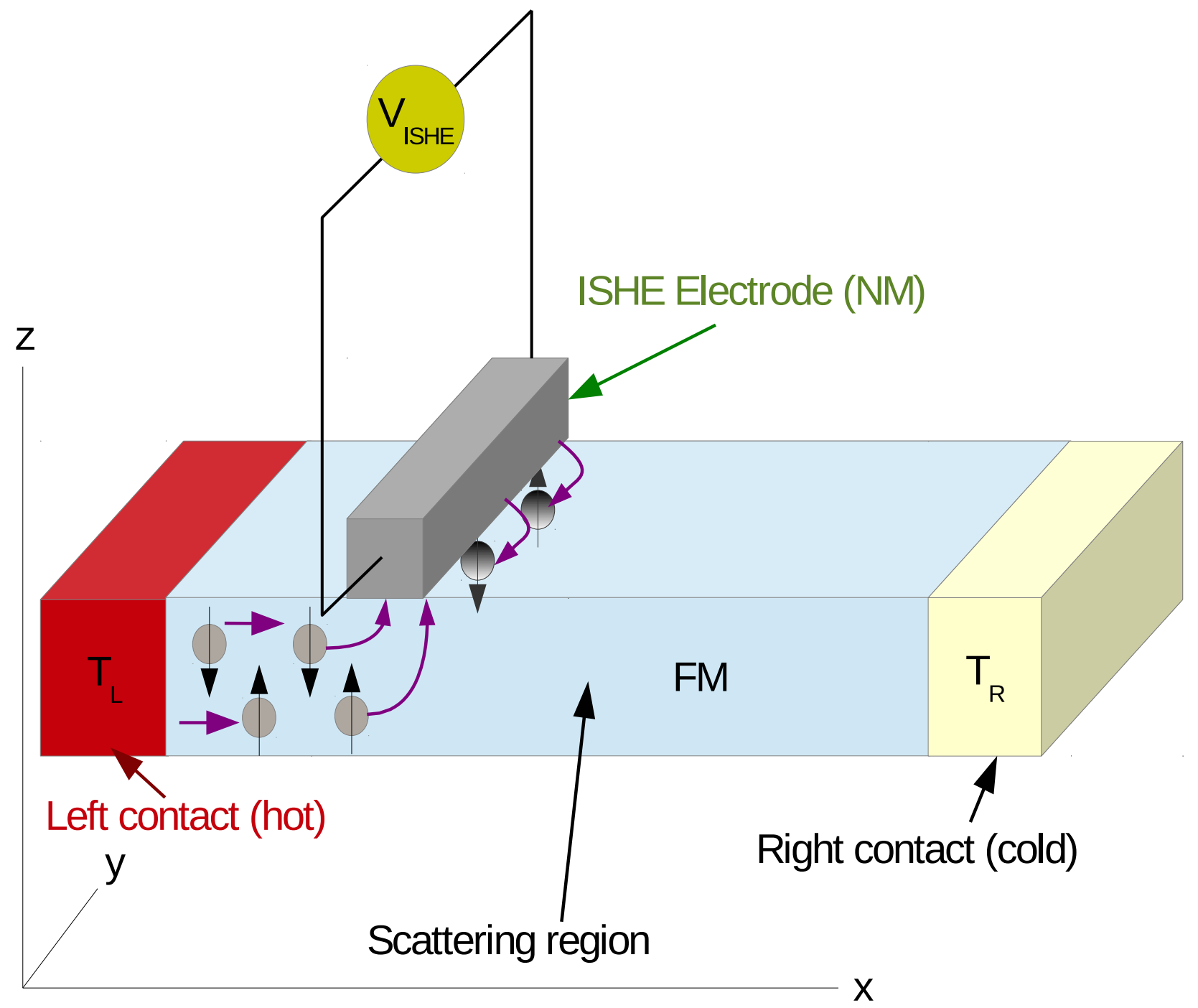

Figure 2.1 A three probe system consisting of left electrode with temperature $\mathrm{T}_{H}$, right electrode with temperature $\mathrm{T}_{C}$ and an ISHE electrode on the scattering region to measure the amount of current from the scattering region into the electrode. 


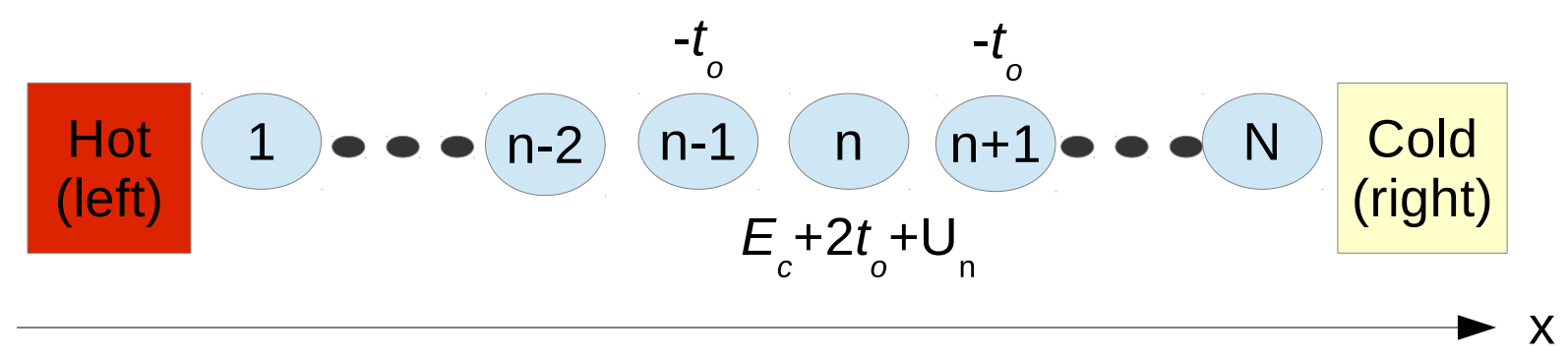

Figure 2.2 Description of the scattering region divided into $\mathrm{N}$ lattice points. The number of grid points $\mathrm{N}$ depends on length of the lattice.

the channel has to be included in Equation 2.5 which now becomes,

$$
[G]=\left[E I-H-U-\Sigma_{l}^{o u t}-\Sigma_{r}^{o u t}\right]^{-1}
$$

The electron density in the scattering region which can be written as $\{\psi\}\{\psi\}^{\dagger}$, generally represented by $\mathrm{G}^{n}$, varies with the applied thermal bias. Using Equation $2.4, \mathrm{G}^{n}$ can be written as $G^{n}=\{\psi\}\{\psi\}^{\dagger}=[G]\{s\}\{s\}^{\dagger}[G]^{\dagger}$. Where $\{s\}\{s\}^{\dagger}$ is written as $\Sigma^{i n}$ (the inflow from the source), $\mathrm{G}^{n}$ can now be written as,

$$
G^{n}=[G] \sum^{i n}[G]^{\dagger}
$$

As there are contacts that interfere with the system, analytical solution cannot be obtained for the Equation 2.7. One way to solve this equation is by expressing the material as finite discrete volumes with points at cell centers as shown in Figure 4.19, and representing those points through a matrix form. In the current research the proposed model will assume isotropic behavior at the cross-section of each lattice point along the scattering region, and assume a 1-dimensional model. At each lattice point (n) along the x-direction, the amount of spin current generated due to the difference in the two spin populations at that position 
can be calculated. This spin current is injected into the attached ISHE electrode at that position which is converted into spin voltage due to the principle of inverse spin Hall effect. In order to calculate the spin current at each lattice point, the population density can be calculated by applying NEGF model to solve Equation 2.7.

The Equation 2.7 represents the fundamental equation behind NEGF formalism. The inflow energy from the contacts occur due to the non-equilibrium thermal difference at the hot and cold ends on the left and right contacts respectively and can be calculated using Equation 2.8. Calculating each of the terms on the right hand side of the Equation 2.7, yields the electron density in the channel,

$$
\begin{gathered}
\Sigma_{l}^{i n}=\Gamma_{l} * f_{l}, \\
\sum_{r}^{i n}=\Gamma_{r} * f_{r}, \\
\Sigma^{i n}=\Sigma_{l}^{i n}+\Sigma_{r}^{i n},
\end{gathered}
$$

where $f_{l}$ and $f_{r}$ are temperature-dependent Fermi-Dirac distribution of the continuous energy states $\epsilon$ at left and right contacts respectively. The Fermi function can be described by Equation 2.9. Here, $\mathrm{T}_{l(r)}$ is the temperature of the contact at left(right) end and $\epsilon$ is the energy. It is through this Fermi-Dirac distribution that an explicit temperature bias is applied to the system.

$$
f_{l(r)}(\epsilon)=\frac{1}{1+e^{\left.\left(\epsilon-\mu_{l(r)}\right) /\left(k_{B} T_{l(r)}\right)\right]}}
$$

When the temperature bias is applied to the scattering region by the end contacts, the conduction electrons tend to move away from the hot contact. The two spin channels (spin-up $(\uparrow)$ and spin-down $(\downarrow))$ associated to electrons move at different rates due to their different effective mass values. The effective mass of the $\uparrow$ and $\downarrow$ conduction electrons depends on the curvature of the respective conduction bands and can be calculated by taking the harmonic mean of the effective masses in the three reciprocal vectors directions in the 
Brillouin zone as given in Equation 4.4.

$$
\begin{array}{r}
E_{\downarrow(\uparrow)}(k)=E_{\downarrow(\uparrow) c x}+\frac{\hbar^{2} * k_{x-\Gamma}^{2}}{2 * m_{\downarrow(\uparrow) x}^{*}}, \\
E_{\downarrow(\uparrow)}(k)=E_{\downarrow(\uparrow) c y}+\frac{\hbar^{2} * k_{y-\Gamma}^{2}}{2 * m_{\downarrow(\uparrow) y}^{*}}, \\
E_{\downarrow(\uparrow)}(k)=E_{\downarrow(\uparrow) c z}+\frac{\hbar^{2} * k_{z-\Gamma}^{2}}{2 * m_{\downarrow(\uparrow) z}^{*}}, \\
m_{\downarrow(\uparrow) \text { conduction }}^{*}=3 *\left[\frac{1}{m_{\downarrow(\uparrow) x}^{*}}+\frac{1}{m_{\downarrow(\uparrow) y}^{*}}+\frac{1}{m_{\downarrow(\uparrow) z}^{*}}\right]^{-1},
\end{array}
$$

where $\mathrm{k}$ is the wavevector and $\mathrm{E}_{\downarrow(\uparrow) c x}, \mathrm{E}_{\downarrow(\uparrow) c y}$ and $\mathrm{E}_{\downarrow(\uparrow) c z}$ are the conduction band edges in the $\mathrm{x}-\Gamma, \mathrm{y}-\Gamma$ and $\mathrm{z}-\Gamma$ directions respectively of $\downarrow(\uparrow)$ electrons. Using the effective mass values of $\downarrow(\uparrow)$ electrons and the respective electronic band gaps, the amount of current generated in the scattering region due to the temperature bias between the contacts can be calculated.

Every electron has multiple energy levels available to accommodate their movement. Depending on the electron's eigen energy, charge transport dominates in certain energy bands which can be calculated from transmission function given by,

$$
\Xi=\operatorname{Trace}\left[\Gamma_{l} G \Gamma_{r} G^{\dagger}\right]
$$

where $\Gamma_{l}$ and $\Gamma_{r}$ are $\mathrm{NxN}$ left and right contact anti-Hermitian matricies of $\Sigma_{l}^{\text {out }}$ and $\Sigma_{r}^{\text {out }}$ respectively that govern the inscattering and outscattering from contacts. All these parameters 
can be calculated from,

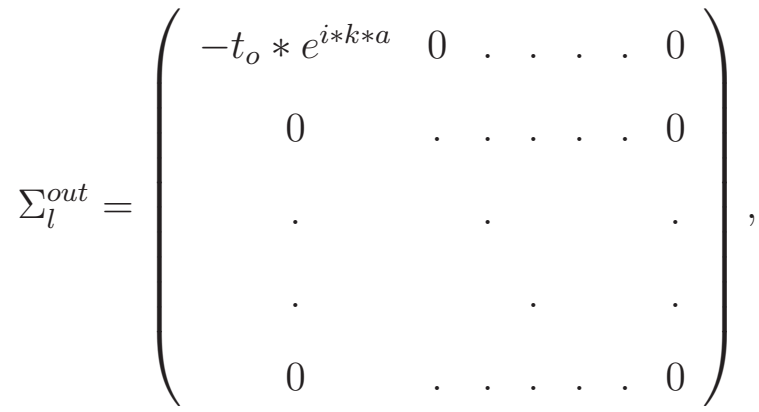

$$
\begin{aligned}
& \Gamma_{l}=i *\left[\Sigma_{l}^{\text {out }}-\Sigma_{l}^{\text {out }}{ }^{\dagger}\right],
\end{aligned}
$$

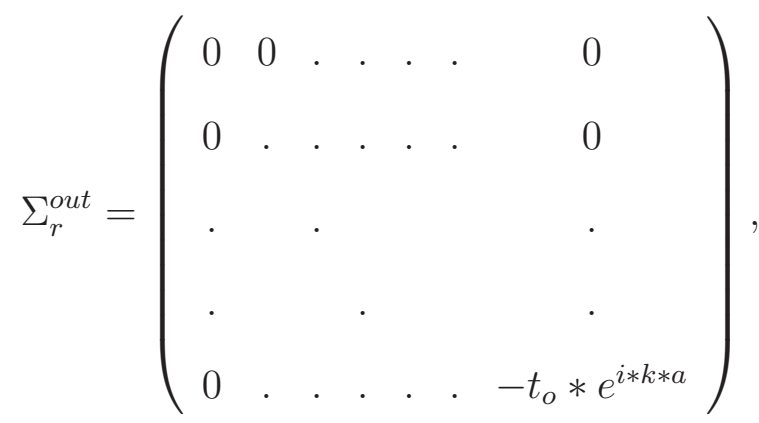

$$
\begin{aligned}
& \Gamma_{r}=i *\left[\Sigma_{r}^{\text {out }}-\Sigma_{r}^{\text {out }}{ }^{\dagger}\right],
\end{aligned}
$$

where $\mathrm{k}$ is $\cos ^{-1}\left(1-\frac{E}{2 t_{o}}\right)$. E is the energy level in the fine spectrum of energy bands, $-t_{o} * e^{i * k * a}$ $t_{o}$ is analytical wavefunction given by,

$$
t_{o}=\frac{\hbar^{2}}{2 * m_{e} * a^{2} * q},
$$

where $\mathrm{a}$ is the distance between cell centers. The distance between the cell centers is also correlated with the energy cut-off. $\mathrm{m}_{e}$ is the conduction electron effective mass calculated from Equation 4.4, q is the charge of an electron, $\hbar$ is the reduced Planck's constant.

The transmission function $(\Xi)$ of an energy band when multiplied with the Fermi functions, gives the conductance for each energy level. By integrating the quantum conductance of an electron in a fine spectrum of energy bands, the overall quantum conductance 
of the electron with that eigen energy can be obtained. Incorporating the same process for all the eigen energy states, the overall quantum conductance in the material lattice can be obtained. The quantum conductance multiplied with $\frac{q^{2}}{h}$ yields the current generated by a electron of one eigen energy. As given in Equation 2.17, taking the sum of total current for all eigen energy states, gives the net current in the scattering region.

$$
I_{\text {tot }}=\sum_{\epsilon=E_{\min }}^{\epsilon=E_{\max }} \frac{q^{2}}{h} \Xi(\epsilon)\left(f_{l}-f_{r}\right) d E
$$

A lattice divided into $\mathrm{N}$ lattice points, as shown in Figure 4.19, can be described by an $\mathrm{NxN}$ matrix called the Hamiltonian matrix. The higher value of $\mathrm{N}$ gives better approximation by increasing the cut off energy while also taking a toll on the computational expense. A 1-D Hamiltonian matrix with $\mathrm{N}$ lattice points, $[\mathrm{H}]_{N * N}$, can be written as given in Equation 2.18. The NxN Hamiltonian matrix has 'N' eigen values which are the eigen energy states of the scattering region. In Equation 2.18, $\mathrm{E}_{c}$ is the conduction band edge that can be obtained from DFT calculations and $t_{o}$ is represented in Equation 2.16. It can be observed that the Hamiltonian matrix of the scattering region depends on the parameters obtained from DFT calculations and the fineness of the grid. 


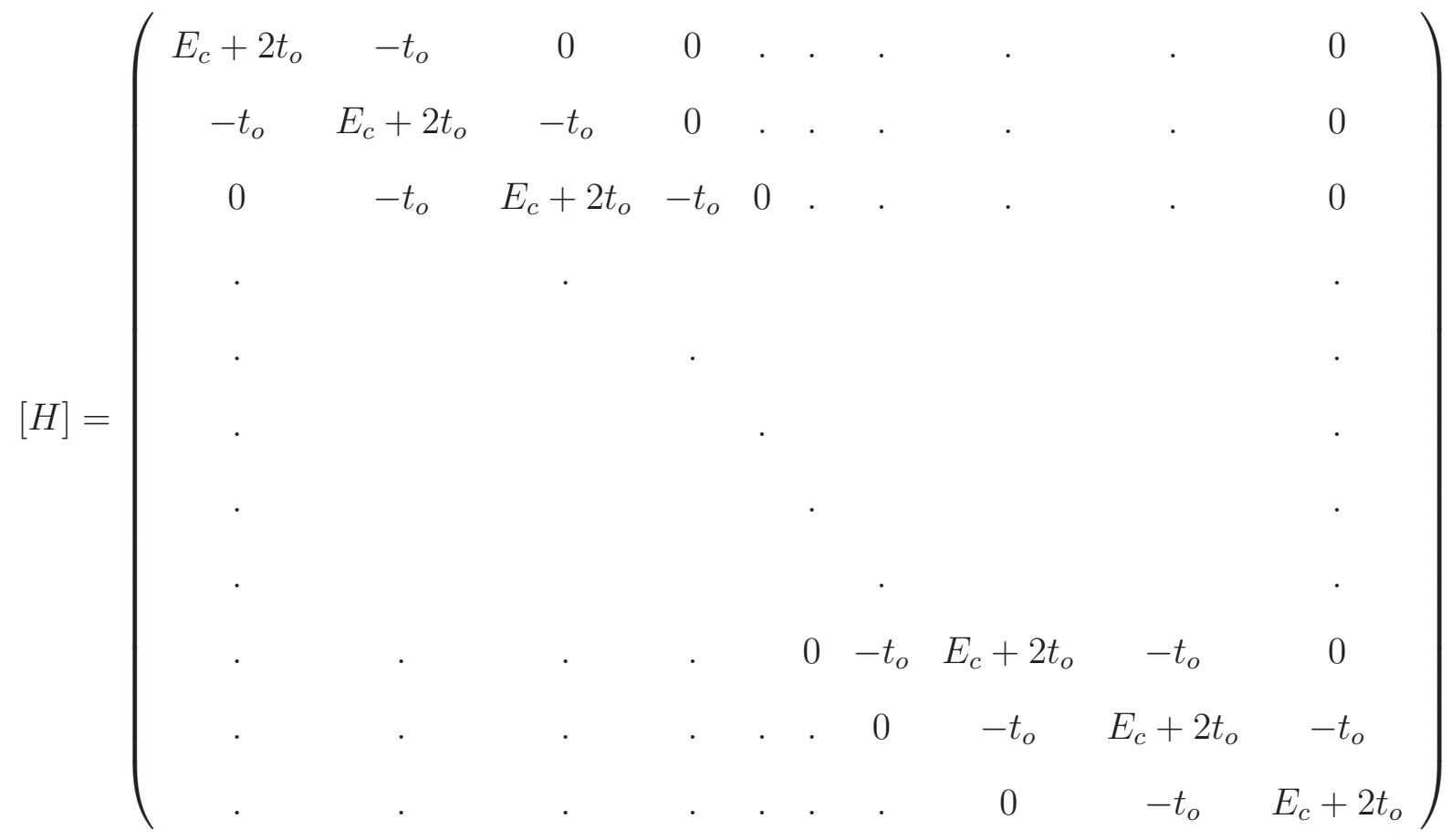

For each energy level, the potential of the scattering channel (U) and the electron density $(\mathrm{N})$ can be calculated iteratively until self-consistency in the system is reached. This is described in the flow chart in Figure 2.3. The self consistent procedure accounts for the electron correlation within each spin channel. After a converged self-consistent estimation of $\mathrm{U}$, the electron density for that eigen energy state, which is the trace of $G^{n}$ matrix, is used to calculate the current generated at that state. As, isotropic conditions are assumed at each lattice point, trace of $G^{n}$ matrix in Equation 2.7 gives electron density per unit area.

Summing the currents over all eigen energy states gives the total current in the scattering region. Using the converged value of $U$ at each energy level, the current in the channel at that respective energy level can be calculated. By summing the currents at all energy levels, the total current in the scattering region can be calculated.

In this research the spins will be treated independent of each other. This commonly is 


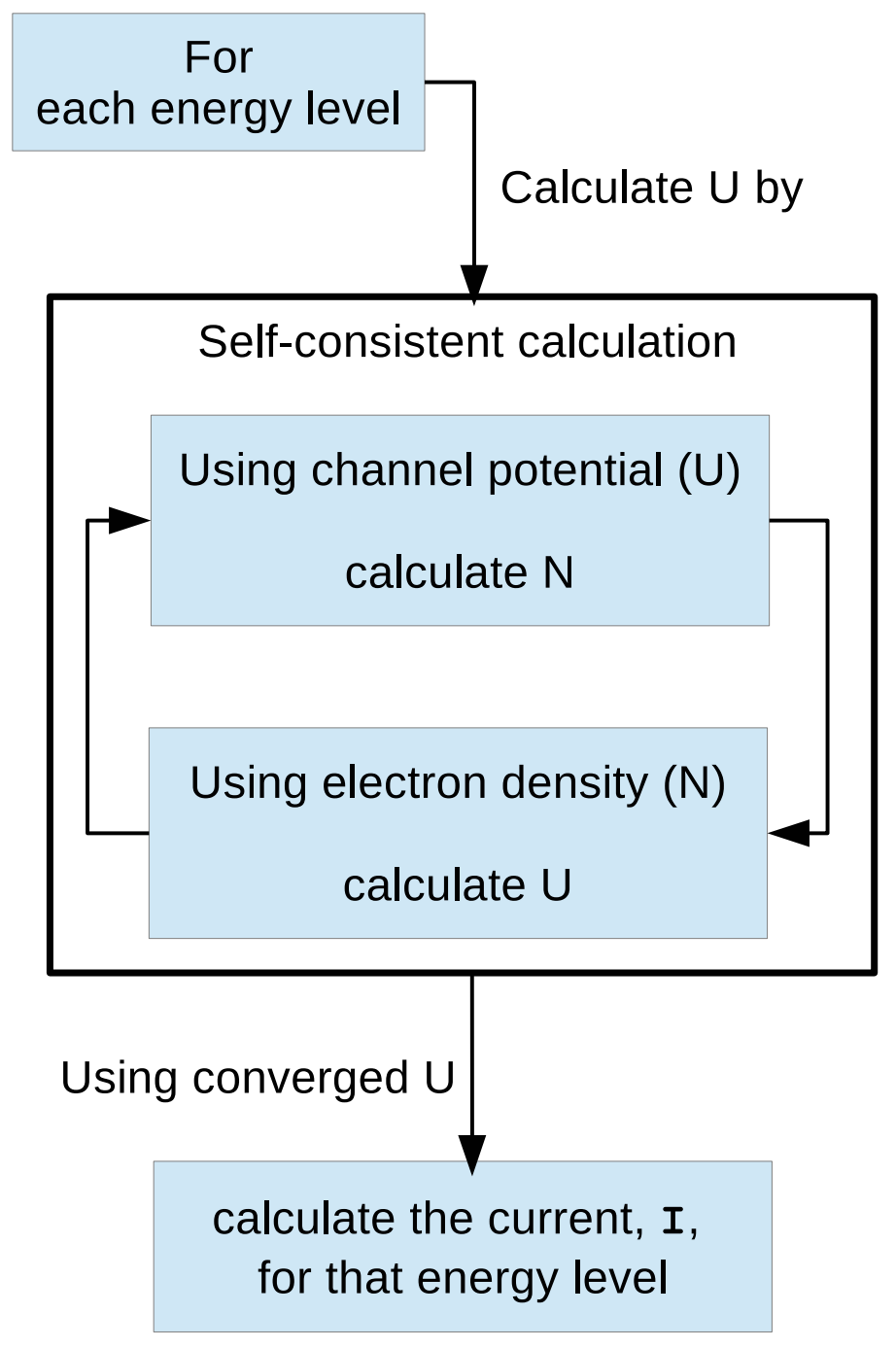

Figure 2.3 NEGF flow chart of the self-consistent method. The self-consistent criteria is when consecutive energy difference reaches below 1e-6.

referred to as the Stoner model [99], visually depicted in Figure 2.4. In doing so, the currents of the spin-up, $\mathrm{I}_{\uparrow}$, and spin-down, $\mathrm{I}_{\downarrow}$, can be obtained from Equation 2.19, for the respective $\Xi_{\uparrow}$ and $\Xi_{\downarrow}$ obtained from Green's formalism. In a ferromagnetic material there is a net spin-polarization present in the lattice. This can lead to spin-polarized charge current under temperature bias. The difference in the Fermi level of spin-up and spin-down conduction electrons in a material causes imbalance between the populations of spin-up and spin-down electrons that can lead to spin-polarized charge current. 


$$
\begin{aligned}
I_{\text {tot }(\uparrow)} & =\sum_{\epsilon=E_{\min }}^{\epsilon=E_{\max }} \frac{q^{2}}{h} \Xi_{(\uparrow)}(\epsilon)\left(f_{l(\uparrow)}-f_{r(\uparrow)}\right) d E \\
I_{\text {tot }(\downarrow)} & =\sum_{\epsilon=E_{\min }}^{\epsilon=E_{\max }} \frac{q^{2}}{h} \Xi_{(\downarrow)}(\epsilon)\left(f_{l(\downarrow)}-f_{r(\downarrow)}\right) d E
\end{aligned}
$$

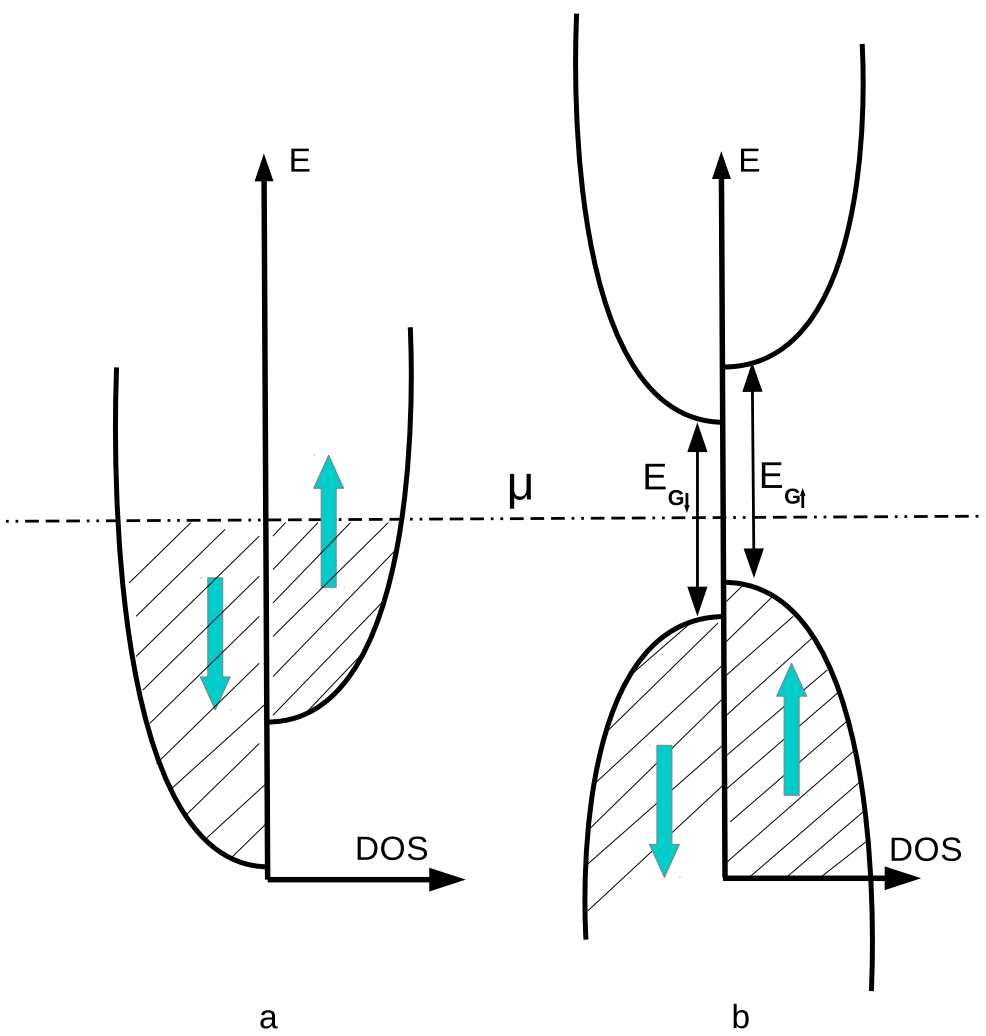

Figure 2.4 An illustration of density of states for spin-polarized ferromagnetic a) Metal b) Semiconductor. The electrochemical potential of the spin-up conduction electrons, $\mu_{\uparrow}$, is different from that of spin-down conduction electrons, $\mu_{\downarrow}$. The difference in the energy gap for spin-up(spin-down) electrons is $E_{G \uparrow(\downarrow)}$. 


\subsubsection{Calculation of the spin Seebeck coefficient}

For $\uparrow, \downarrow$ components, when a temperature gradient $(\Delta \mathrm{T})$ is applied, the spin Seebeck coefficient can be written as $\mathrm{S}_{\uparrow(\downarrow)}=\frac{V_{I S H E \uparrow(\downarrow)}}{\Delta T}$. Figure 2.5 shows a model I-V ISHE curve for spin-up and spin-down currents that can be obtained in a ferromagnetic material.

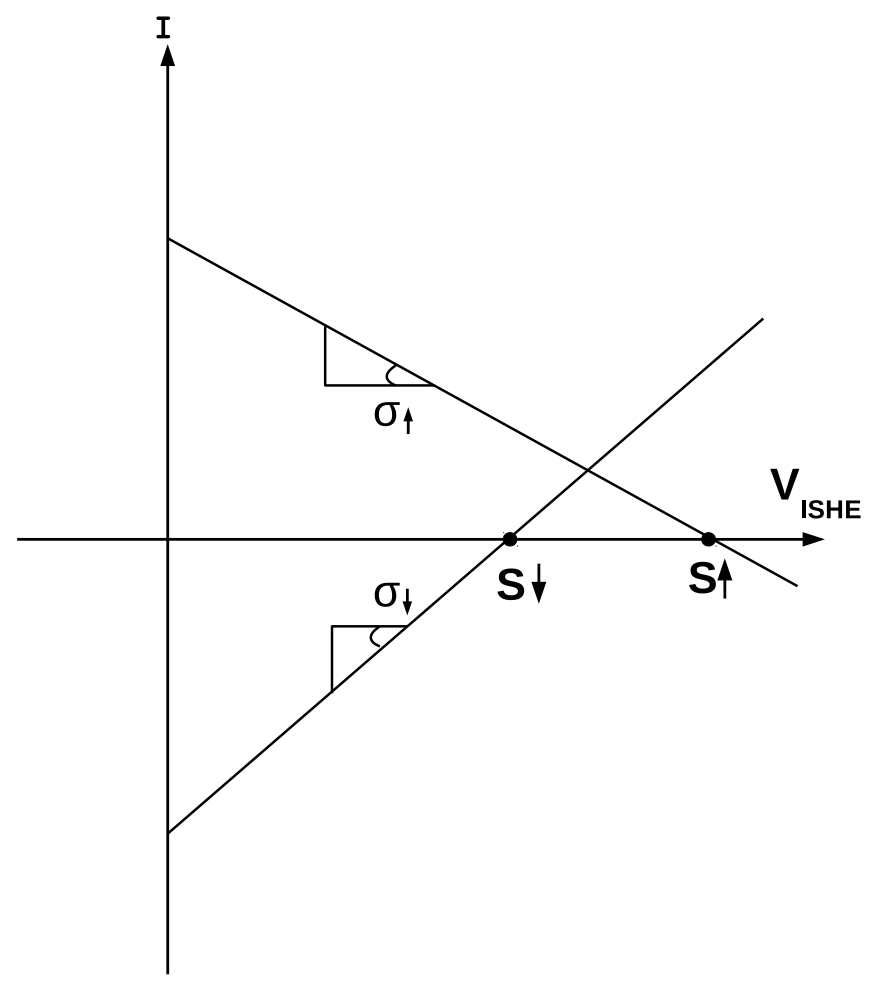

Figure 2.5 Varying $V_{I S H E}$ to neutralize spin-up and spin-down currents separately in the scattering region, gives the respective spin Seebeck coefficients $S_{\uparrow}, S_{\downarrow}$.

A voltage in the reverse bias will be applied to the ISHE electrode $\left(V_{I S H E}\right)$, Figure 2.1, which will inject spin current into the ferromagnetic (FM) material (scattering region in the current model), that opposes the spin current generated in the FM due to temperature bias. 
This $\left(V_{I S H E}\right.$ will be iterated until current flow is zero between the left contact and ISHE electrode. The respective slopes in the IV curve, Figure 2.5, gives conductivity $(\sigma)$ of spinup and spin-down electrons. The $V_{I S H E}$ for which $I_{\uparrow}$, from Equation 2.19, is zero gives the

$V_{I S H E \uparrow}$ and the $S_{\uparrow}$ at that temperature would be $\mathrm{S}_{\uparrow}=\frac{V_{I S H E \uparrow}}{\Delta T}$. A similar iterative procedure can yield $\mathrm{S}_{\downarrow}$. The electronic contribution to thermal conductivity $k_{e}$ can be calculated from Fourier's law, $k_{e}=\frac{I_{\uparrow}+I_{\downarrow}}{A} \frac{\Delta x}{\Delta T}$.

The presence of temperature gradient along the ferromagnetic material changes the density flux of spin-up and spin-down electrons. Exploring the substitutional effects to improve the difference in this spin-up and spin-down density flux will make a material suitable for spin caloritronic applications. In this $\mathrm{PhD}$ work, the effect of substitutions on the spin transport of Cobalt spinel oxides will be studied, to elucidate their behavior in the presence of temperature gradient and test their candidature as spin caloritronic materials.

\subsubsection{Grid size and convergence}

Choosing an apt grid size for calculations is necessary to get consistent and reliable trend. The Figure 2.6 shows the variation of population density with the increase in grid points in the scattering region. The population density reaches a value of $7^{*} 10^{15}$ starting from 300 grid point fine mesh in a 36nm scattering region. But with the increase in the mesh size the computational time increases exponentially reaching 9 hours to complete a calculation when initiated on an 8 processors. In Figure 2.6, a grid size of 200, which represents $90 \%$ of the converged value, can be chosen without compromising on the quality of the data. Hence for each Hamiltonian a convergence study was performed and apt grid size was chosen for calculations. 


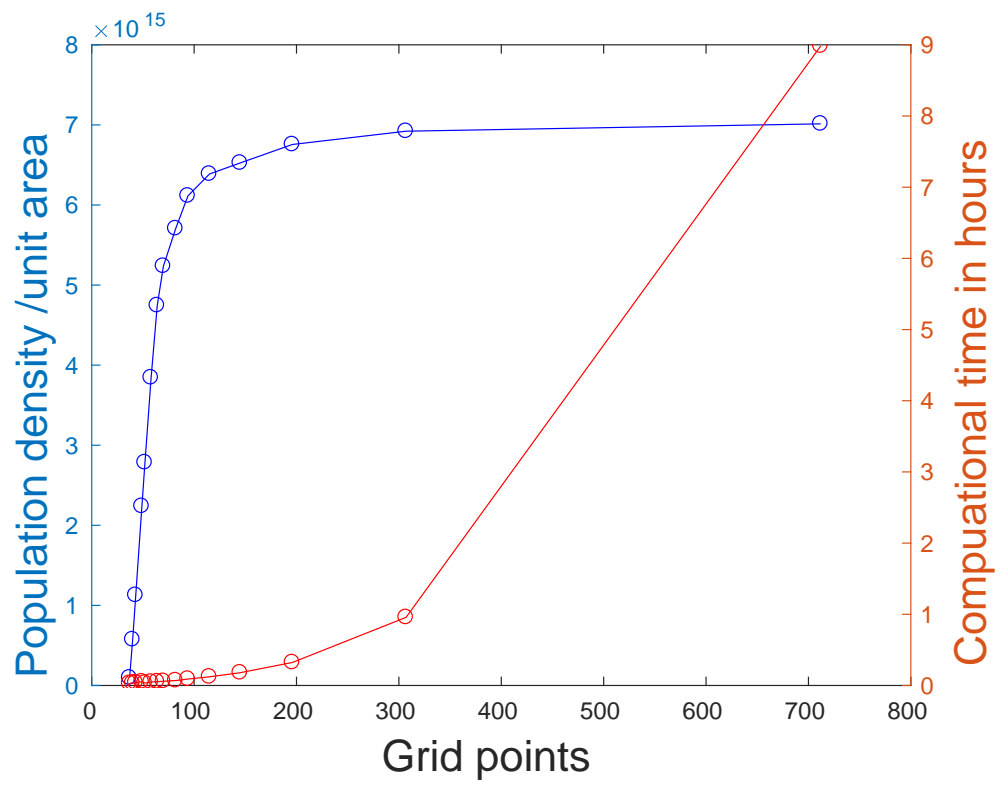

Figure 2.6 Increasing the grid size to see variation in the population density in the scattering region, given in blue color. For each grid point the respective computational time consumed is shown in red. 


\section{CHAPTER 3}

\section{THEORY OF TRANSVERSE SPIN SEEBECK EFFECT}

Spin caloritronics is a transpiring field with immense potential in heat conversion applications. The interplay between the temperature gradient and charge transfer in spinpolarized materials can be explored to extract high inverse spin Hall voltage across an attached metal electrode on the surface. This is the operation principle of spin Seebeck effect.

When a temperature gradient $(\nabla \mathrm{T})$ is applied, the $\uparrow(\downarrow)$ components move away from

hot source at different rates and hence have different spin Seebeck coefficients, $\mathrm{S}_{\uparrow(\downarrow)}$ causing a spin redistribution across the material lattice. The presence of temperature gradient along the ferromagnetic material changes the density flux of spin-up and spin-down electrons. Exploring the substitutional effects to improve the difference in this spin-up and spin-down

density flux will make a material suitable for spin caloritronic applications. Sometimes the substitutions make the material spin-polarized while also making the material conductive which hampers the application of the material for thermoelectrics. Hence theoretical characterization of a material performance for spin caloritronic applications can be made using the developed model in this research.

\subsection{Spin Current Across the Magnetic and Non-Magnetic Interfaces}

At each location along the $\mathrm{x}$ axis, in Figure 4.19, different amount of spin current is injected into the NM contact from the FM material. In a conventional Seebeck effect, the temperature difference between the junctions of two dissimilar metal joints generates a net voltage. Likewise, in case of spin Seebeck effect (SSE), the difference in the temperature 
between the magnons (electron spin waves) in the ferromagnetic film (considered to be close to that of the FM temperature) and the electrons in the attached NM contact (considered to be close to that of the NM temperature), generates an inverse spin Hall voltage in the NM contact.

As SSE was observed even in magnetic insulators, the Spin Seebeck coefficient cannot be fully expressed as a sole function of conduction electrons. Hence, a microscopic theory proposed by Xiao et al [85] was employed in this research. As in conventional Seebeck effect where the temperature difference between the left and right contacts drives the charge current, here the temperature difference between the FM $\left(\mathrm{T}_{F}\right)$ and $\mathrm{NM}\left(\mathrm{T}_{N}\right)$ drives the spin current across the interface of FM and NM. The model approximates that the difference between the $\mathrm{T}_{F}$ and $\mathrm{T}_{N},\left(\Delta T=T_{F}-T_{N}\right)$, changes from a positive value at the hot end to a negative value at the cold end, flipping the sign at the center which in close approximation to the experimental findings [67].

The spin pumping current from FM to NM occurs due to thermal non-equilibrium between the two, given by Equation 3.1. The thermal spin pumping current $\mathrm{J}_{s p}$ from FM to $\mathrm{NM}$ is proportional to $\mathrm{T}_{F}$ and spin current fluctuations $\mathrm{J}_{f}$ from $\mathrm{NM}$ to $\mathrm{FM}$ is proportional to $\mathrm{T}_{N}$, given by Johnson-Nyquist [100]' [101]. Only the real component of the $\mathrm{J}_{s p}$ contributes to the net $\mathrm{J}_{s}$ towards the NM because the imaginary component averages to zero. The real part of the spin current from FM into the NM as extracted from literature [102] is given by,

$$
J_{s p}=\frac{\hbar}{4 \pi}\left[g_{r} \boldsymbol{m} \times \dot{\boldsymbol{m}}\right]
$$

where $\hbar$ is the reduced Plank's constant, $g_{r}$ is the real part of spin mixing conductance, $\boldsymbol{m}$ is the unit vector that is parallel to the manetization in the material, $\dot{\boldsymbol{m}}$ is the rate of change of magnetization due to thermally activated dynamics in magnetization in FM. To compensate the energy transfer from FM into $\mathrm{NM}$ in the form of spin, a fluctuating spin current, $\mathrm{J}_{f}$, 
flows from NM to FM, as extracted from literature [100]' [101] is given by,

$$
J_{f}=\frac{-M_{s} V}{\gamma}\left[\gamma \boldsymbol{m} \times \boldsymbol{h}^{\prime}\right]
$$

where $\mathrm{M}_{s}$ is the saturation magnetization in the FM, V is the FM volume, $\gamma$ is the electron gyro-magnetic ratio and $\boldsymbol{h}^{\prime}$ is the resultant magnetic field.

Hence, the non-equilibrium thermal difference between FM and NM causes the net spin current, $\mathrm{J}_{s}$, from FM to NM in the z-direction given by $\mathrm{J}_{s}=\mathrm{J}_{s p^{-}} \mathrm{J}_{f l}$

$$
\boldsymbol{J}_{s}=\frac{M_{s} V}{\gamma}\left[\alpha^{\prime}\left\langle m_{x} \dot{m}_{y}-m_{y} \dot{m}_{x}\right\rangle-\gamma\left\langle m_{x} h_{y}^{\prime}-m_{y} h_{x}^{\prime}\right\rangle\right]
$$

where $\alpha^{\prime}$ is the damping enhancement due to spin pumping given by $\gamma \hbar g_{r} / 4 \pi M_{s} V$, the $\mathrm{x}$ and y subscripts of $\boldsymbol{m}$ and $\dot{\boldsymbol{m}}$ are the respective components along x and y axes respectively (see Figure 2.1). Using mean square deviation $\langle\boldsymbol{m} \dot{\boldsymbol{m}}\rangle$ and $\left\langle\boldsymbol{m} \dot{\boldsymbol{h}}^{\prime}\right\rangle$ can be approximated by Equation 3.4 and Equation 3.5 respectively as extracted from Xiao et al [85].

$$
\begin{gathered}
\left\langle\dot{m}_{i}(t) m_{j}(0)\right\rangle=\frac{-\sigma^{2}}{4 \pi \alpha} \int\left[\chi_{i j}(\omega)-\chi_{i j}^{*}(\omega)\right] e^{i \omega t} d \omega \\
\left\langle m_{i}(t) h_{j}^{\prime}(0)\right\rangle=\frac{-\sigma^{\prime 2}}{2 \pi \gamma} \int \chi_{i j}(\omega) e^{i \omega t} d \omega
\end{gathered}
$$

$\chi(\omega)$ is the transverse dynamic susceptibility matrix given by Equation 3.6,

$$
\chi(\omega)=\frac{1}{\left(\omega_{o}-i \alpha \omega\right)^{2}-\omega^{2}}\left[\begin{array}{cc}
\omega_{o}-i \alpha \omega & -i \omega \\
i \omega & \omega_{o}-i \alpha \omega
\end{array}\right]
$$

where $\omega_{o}=\gamma H_{\text {eff }}$ is the FM resonance frequency. $H_{\text {eff }}$ is the external magnetic field applied along the direction of thermal bias that causes saturation magnetization in the material.

Hence, by plugging Equations 3.5 and 3.4 into Equation 3.3 and rearranging for the 
spin current transferred from FM to NM in the z direction as given in literature [85] will be,

$$
\left\langle J_{s}\right\rangle=\frac{\gamma \hbar g_{r} k_{B}}{2 \pi M_{s} V}\left(T_{F M}-T_{N M}\right)
$$

\subsection{Spin Hall voltage}

The factor $\frac{\gamma \hbar g_{r} k_{B}}{2 \pi M_{s} V}$ is called interfacial spin Seebeck coefficient represented by $\mathrm{L}_{s}^{\prime}$. The Spin Seebeck voltage can be calculated based on the spin Hall current in the NM that is generated due to inverse spin Hall effect [103]. The DC spin Hall current in the attached $\mathrm{NM}$ (Pt in this case) along y-direction is,

$$
J_{c}(x) \hat{\boldsymbol{y}}=\theta_{H} \frac{2 e\left\langle J_{s}\right\rangle}{\hbar A} \hat{\boldsymbol{z}} \times \hat{\boldsymbol{x}}
$$

where $\theta_{H}$ is the spin Hall angle of NM contact (Pt in this case). The Inverse spin Hall voltage generated at location $\mathrm{x}$ is given by Equation 3.9,

$$
V_{I S H E}(x)=\rho l J_{c}(x)=\frac{l * J_{c}(x)}{\sigma}
$$

where $\rho$ is the electrical resistivity, $l$ is the length of the NM contact and $\mathrm{J}_{c}(\mathrm{x})$ is the spin current from FM to NM as calculated in Equation 3.8. 


\section{CHAPTER 4}

\section{INVERSE SPIN HALL VOLTAGE IN LANTHANUM SUBSTITUTED YTTRIUM IRON GARNET}

Spin Seebeck theory and NEGF transport theory combined with DFT can be used to theoretically calculate the spin voltage in the attached NM metal on the scattering region. As a model has been developed in this research, validating this model with the available experimental data is necessary.

The experimental investigations reported for transverse spin Seebeck are not many in comparison to longitudinal spin Seebeck effect. The reason being that longitudinal spin Seebeck based thermal energy extraction device fabrication is simpler when compared to a transverse spin Seebeck device fabrication. Transverse spin Seebeck based energy extraction though complicated in design, can enhance the overall voltage output when compared to the longitudinal configuration. As this research is focused on transverse mode of operation as discussed in Chapter 1, the implemented theoretical model was first compared to the experimental data available on transverse configuration of YIG based device. Once the model was validated, it was used to demonstrate its applicability to new design spaces.

To calculate the transverse spin Seebeck effect in ferromagnetic material lattices, a spin transport model, as described by Xiao et al [85], was employed. To implement this model to the unknown design space formed by cobalt spinel materials, several parameters have to be optimized to match the available experimental data. In the literature, transverse spin Seebeck configuration was investigated on ferrimagnetic insulators like lanthanum substituted Yttrium Iron Garnet (La:YIG) [67]. This material has drawn attention due to the 
spin transfer capability in the presence of temperature gradient across the lattice, as shown in Figure 4.1.

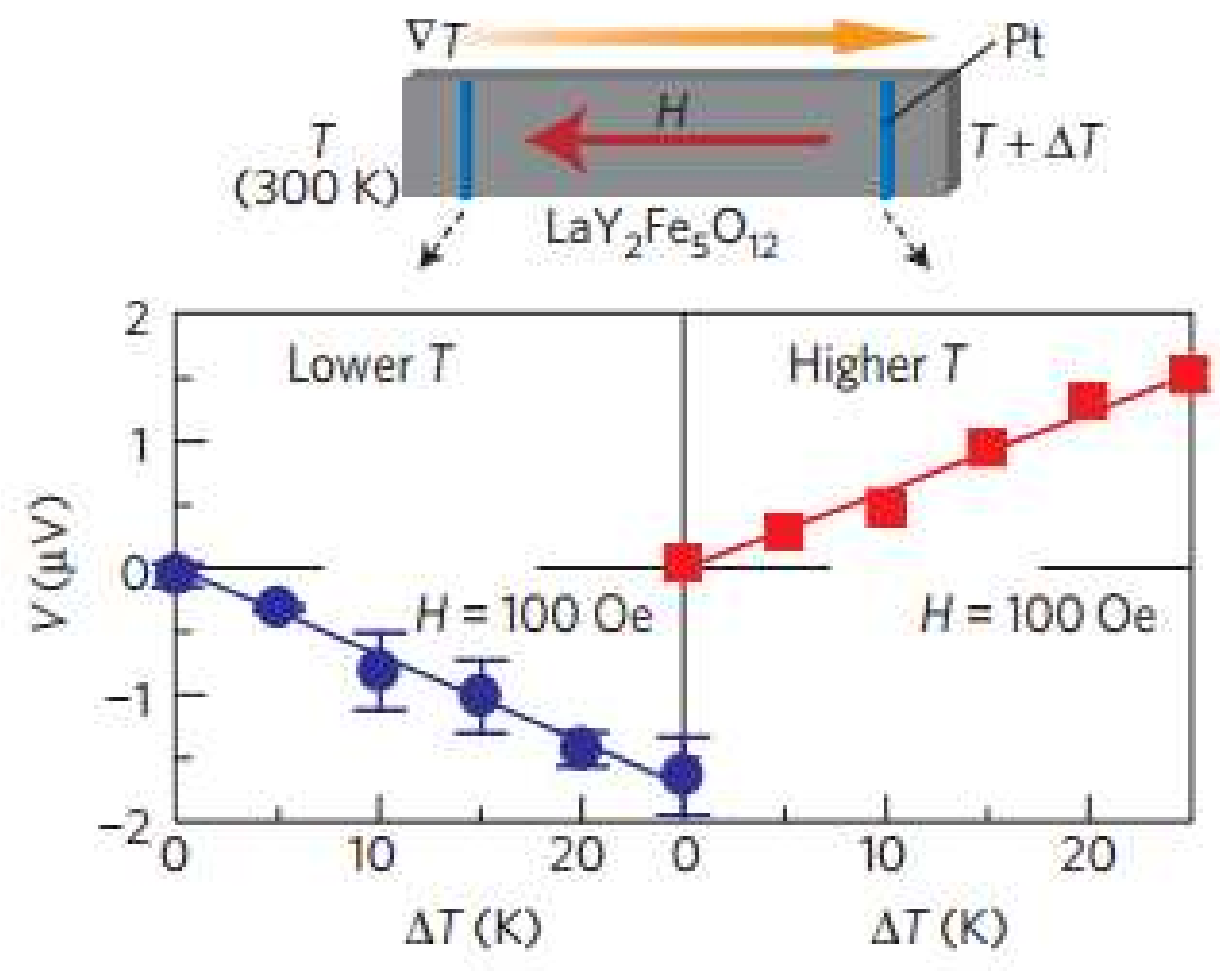

Figure 4.1 Experimental demonstration of spin seebeck effect in La:YIG at various lattice points [67].

Experimental trend from literature, as shown in Figure 4.1, depicts the linear increase in the inverse spin-Hall voltage with the increase in the temperature difference at the ends as reported by Uchida et al [67] in 2010. In their study, Pt electrode are placed at the longitudinal ends of the material to extract the maximum spin induced voltage. Specifically the transverse spin conversion arrangement has also displayed a linearly increasing voltage when measured along the device length as shown in Figure 4.2. It is interesting to note that the voltage signal changes its sign at the centre. This trend was reproduced using the NEGF 
based spin transport model, as will be discussed later in this chapter.

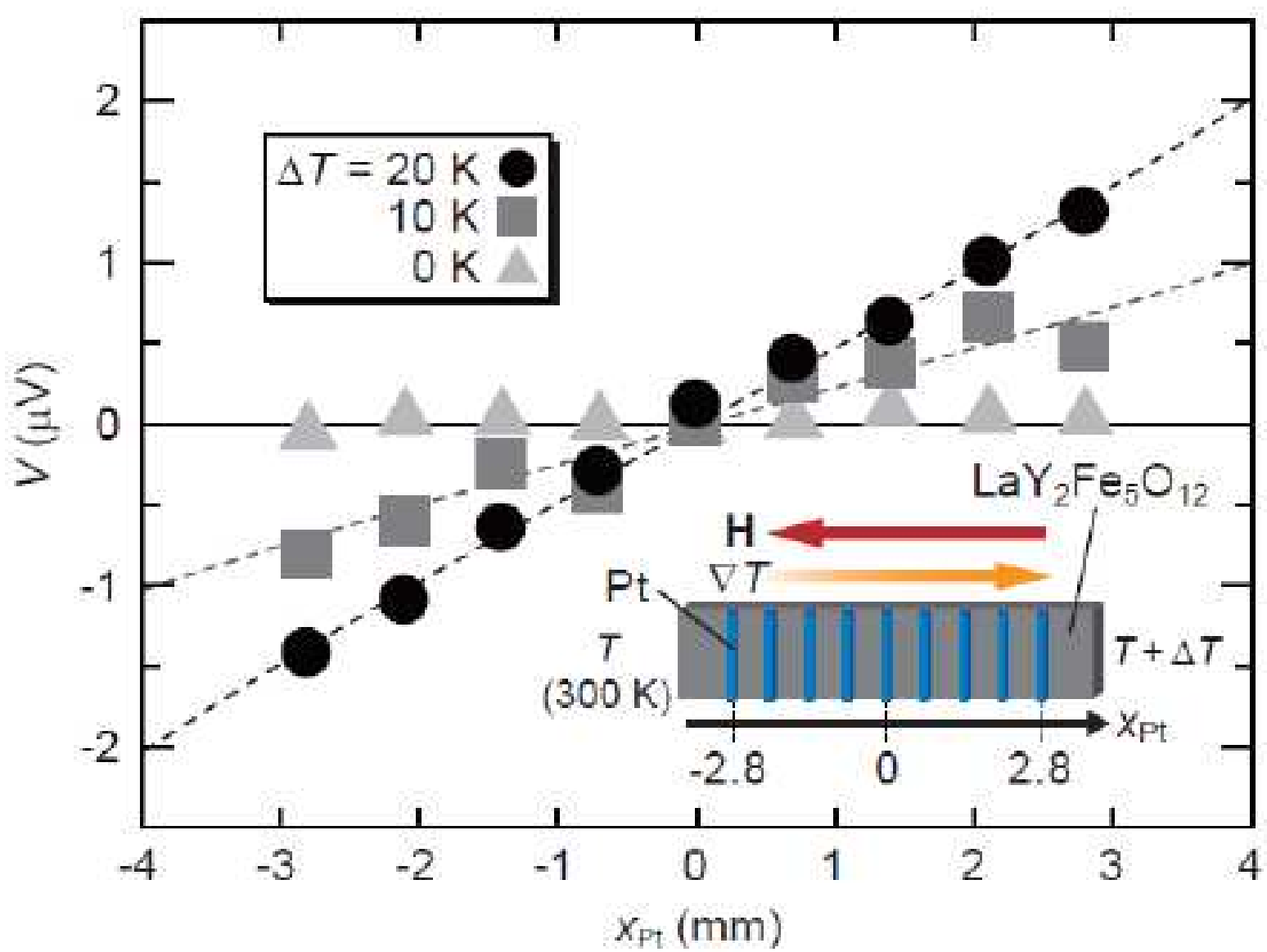

Figure 4.2 Spin voltage induced into Pt contacts at various locations along the La:YIG device [67]

Transverse spin Seebeck configuration, which is the major scope of this research, has been experimentally verified using La:YIG [67] and NiFe [89]. Insulating magnetic materials like La:YIG displaying spin Seebeck effect is a strong indication of magnaon driven spin Seebeck that is caused due to spin redistribution in a material and also proves the capability of spin carriers in a material lattice to generate voltage. In addition to this, semiconducting or insulating oxide magnetic materials have immense scope in this emerging field due to their ability to accommodate wide variety of substitutions and tune various electronic and magnetic properties. Hence we chose La:YIG as our material of study to validate and ver- 


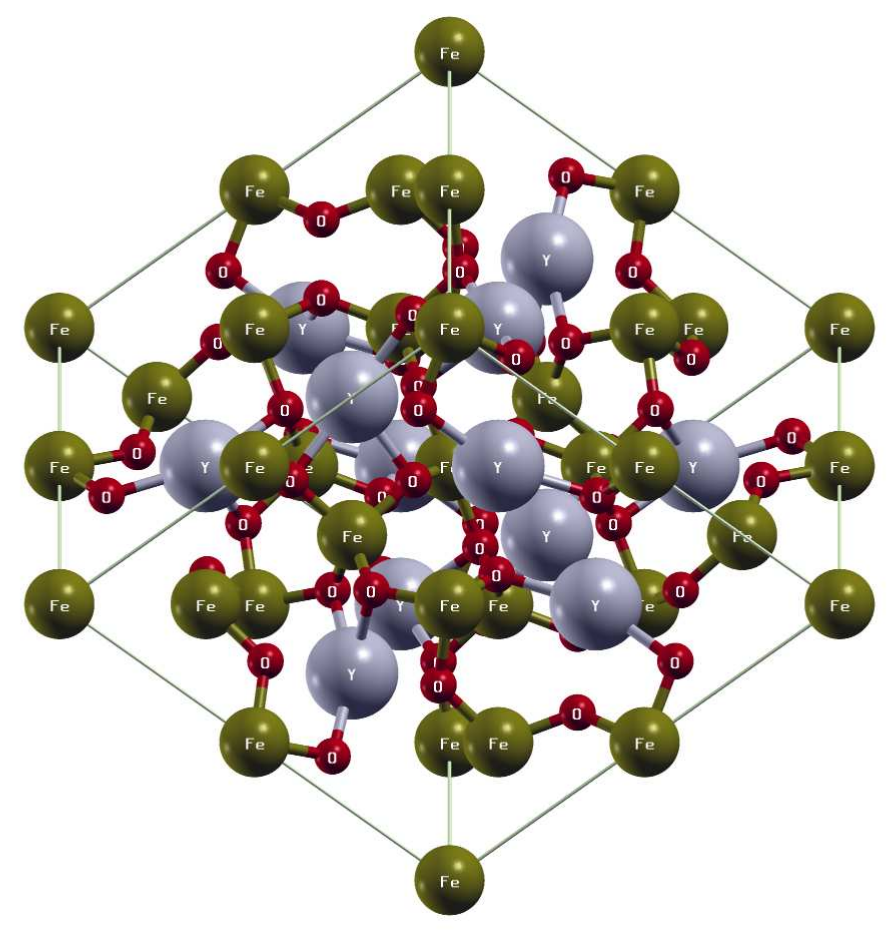

Figure 4.3 A 80 atom super cell of YIG. The green atoms represent $\mathrm{Fe}^{+3}$ ions, the gray atoms represent $\mathrm{Y}^{+3}$ ions and the red atoms represent $\mathrm{O}^{-2}$ ions.

ify the developed model. $\mathrm{LaY}_{2} \mathrm{Fe}_{5} \mathrm{O}_{12}$ (La:YIG) material lattice is obtained by substituting one Yttrium with Lanthanum in $\mathrm{Y}_{3} \mathrm{Fe}_{5} \mathrm{O}_{12}$ (YIG). There have been few theoretical studies reporting the fundamental property study using DFT to calculate lattice parameter, electronic band structure and effective mass data for YIG [90], [91], [92] but there is no available electronic band structure data for La:YIG. Hence, first principle calculations based of DFT were performed on YIG to compare the effective mass and band gap data with literature available for YIG, and a similar approach has been applied to calculate the electronic band structure data for La:YIG. 


\subsection{Fundamental parameters of YIG}

A primitive $\mathrm{Y}_{3} \mathrm{Fe}_{5} \mathrm{O}_{12}$ unit cell has 20 atoms. A 2x2x1 super cell, Figure 4.3, was constructed that comprises 80 atoms. The Fe atoms with tetragonal and octahedral coordination carry majority of the magnetic moment of YIG while Y atoms having dodecahedral coordination and $\mathrm{O}$ atoms have little contribution to the magnetic moment. Fe atoms have highest number of unpaired d electrons that contribute to magnetic moment in the material. The electronic structure of YIG was calculated by implementing the scheme discussed in the computational details section in the previous chapters of this thesis. The computed lattice constant of YIG of $11.62 \AA$ matches well with the available experimental data of $12.39 \AA$ [92].

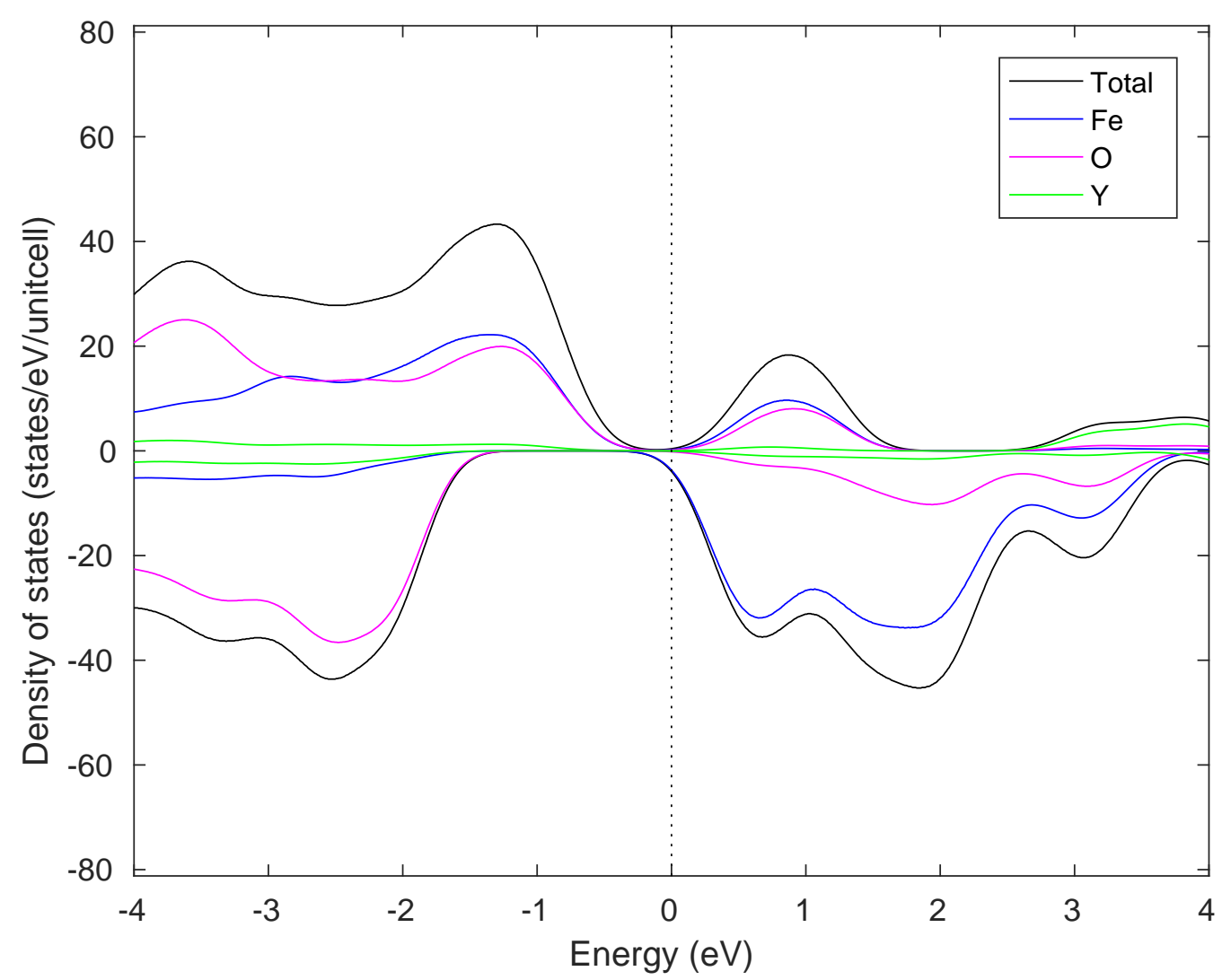

Figure 4.4 The atom projected density of states of iron, yttrium and oxygen show their contributions towards the total density of states near the Fermi region in the YIG material lattice. The positive density of states represent the contribution due to spin up electrons and the negative density of states represent the contribution due to spin down electrons. 
Figure 4.4 shows the calculated total density of states of pure YIG represented by the formula $\mathrm{Y}_{3} \mathrm{Fe}_{5} \mathrm{O}_{12}$. The immediate states below Fermi energy level are contributed by spin up states of $\mathrm{O}^{-2}$ and $\mathrm{Fe}^{+3}$ ions. And the available states above fermi are due to spin down electrons from $\mathrm{Fe}^{+3}$ ions in the tetrahedral sites. This material clearly shows a ferrimagnetic characteristics evident from the charge transfer from spin up states of valance bands to spin down states of conduction band.

The valence and conduction bands of spin up and spin down bands of YIG is shown in Figure 4.5 and Figure 4.6. The spin down contribution to the bands is given in red, the spin up contribution to bands is given in black and the combined valence and conduction bands for the YIG material is shown in Figure 4.7. The k-point path was chosen along the three reciprocal lattice vector directions. $\chi(0,0,1 / 2), \Gamma(0,0,0), \mathrm{L}(1 / 2,0,0)$ and $\eta(0,-1 / 2,1 / 2)$ represent the symmetry k-points. $\chi$ to $\Gamma, \Gamma$ to $\mathrm{L}$ and $\Gamma$ to $\eta$ represent the three reciprocal vector directions. The $\uparrow$ and $\downarrow$ channels have a band gap of $1.1689 \mathrm{eV}$ and $2.2287 \mathrm{eV}$ respectively suggesting that spin up channel is more conductive than the spin down channel. In the combined bands of the YIG, the spin up channel's valance band and spin down channel's conduction band contribute to the majority of the charge transport. In the presence of thermal gradient, the conduction band that corresponds to the $\downarrow$ channel contributes to the electron movement creating a non-equilibrium in spin distribution along the lattice.

The band gap of the YIG lattice calculated from the Ab intio calculations was $0.3262 \mathrm{eV}$ which is corroborated with values reported in the literature [90], where a band gap of $0.33 \mathrm{eV}$ was reported. As discussed in the previous section, each spin channel is treated independently. As spin down channel's conduction band contributes to the conduction band of the YIG material, its effective mass plays a vital role in calculating electron transport. The effective mass of the spin down conduction band was calculated as shown in Figures 4.8, 4.9 and 4.10. The effective mass values in the three reciprocal vector direc- 


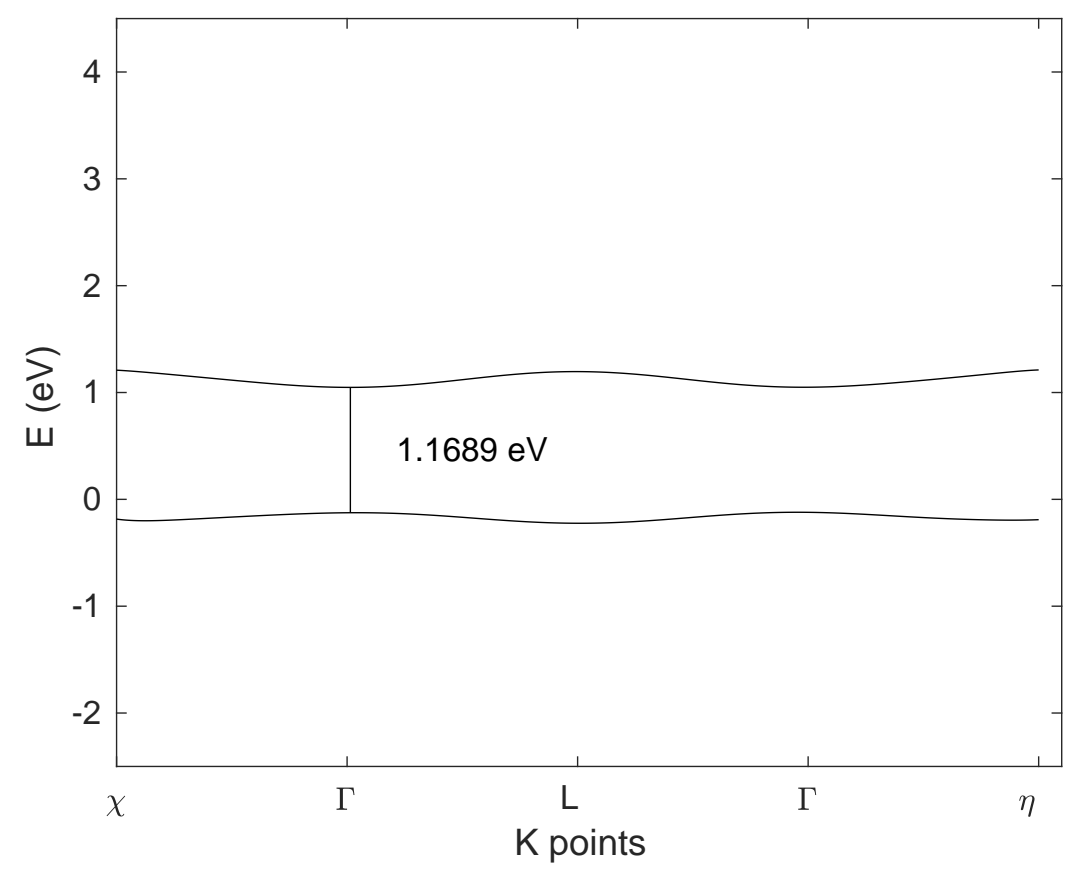

Figure 4.5 The spin up channel of YIG material

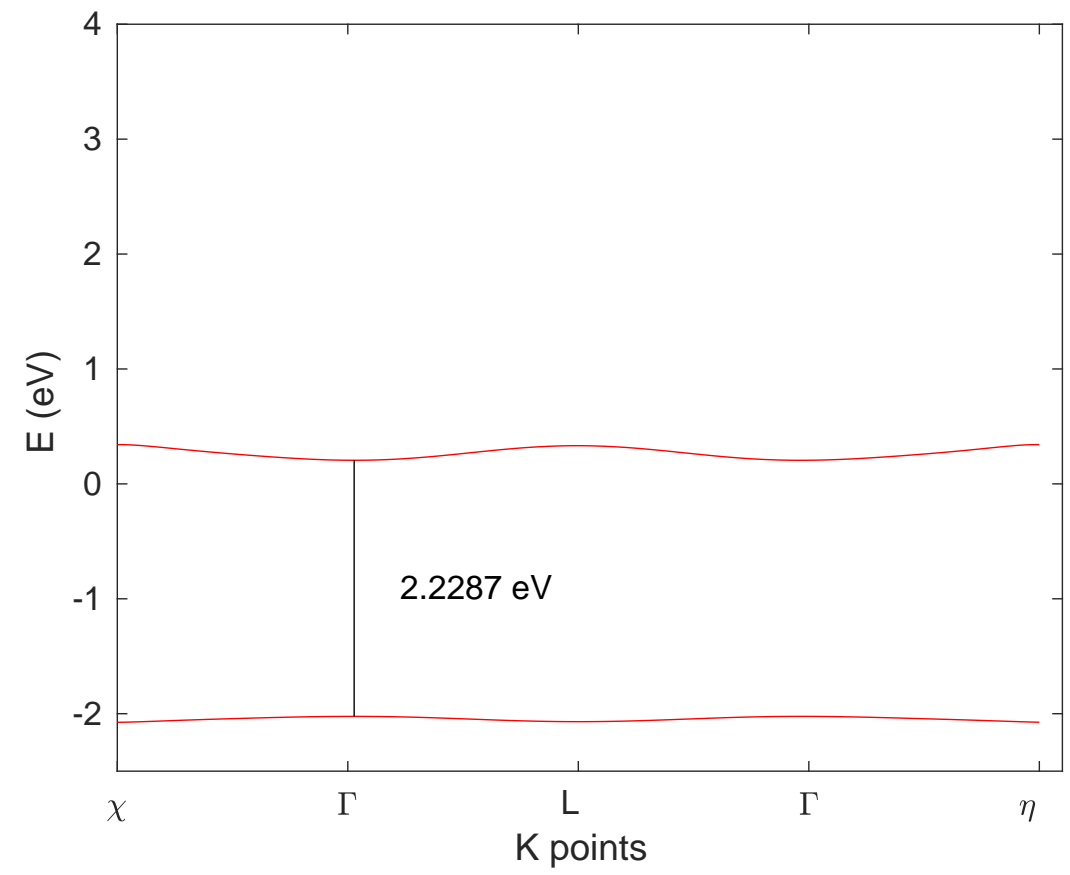

Figure 4.6 The spin down channel of YIG material 


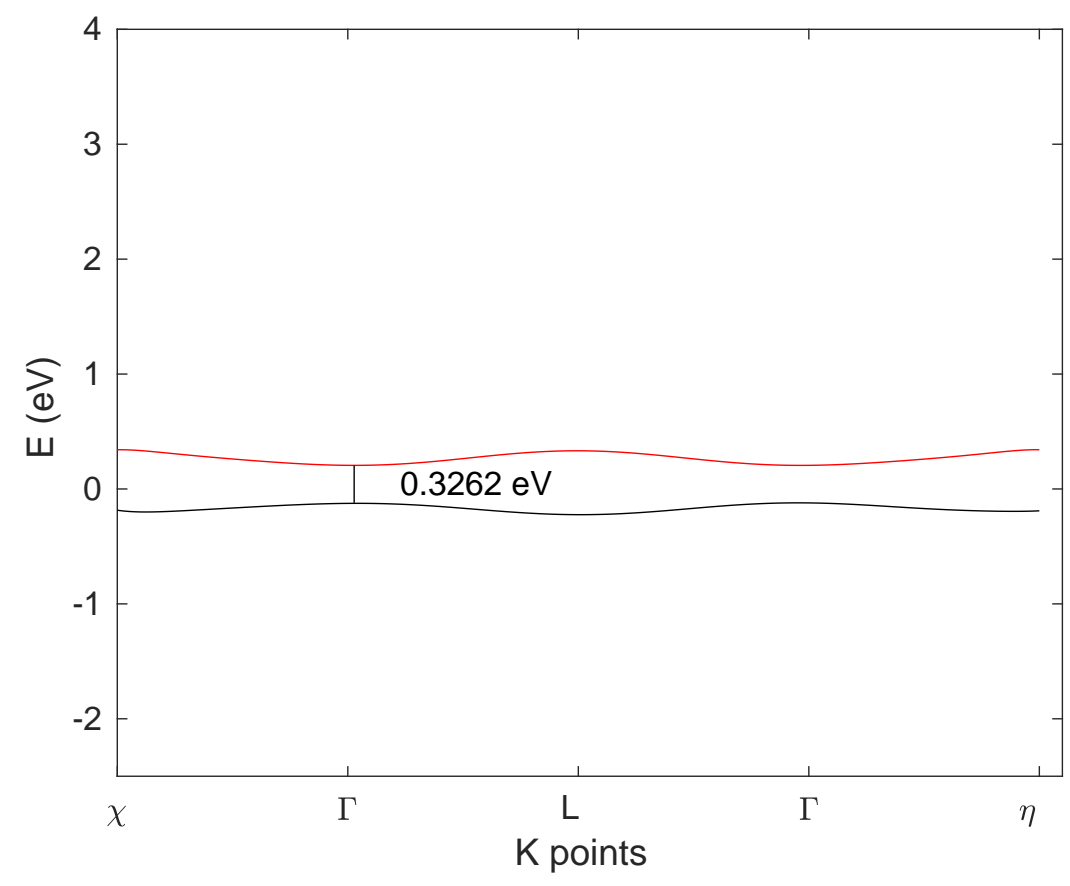

Figure 4.7 The valence band corresponds to the spin up channel and the conduction band corresponds to the spin down channel of YIG material.

tions $\chi-\Gamma\left(\mathrm{m}_{x}\right), \Gamma$ to $\mathrm{L}\left(\mathrm{m}_{y}\right)$ and $\Gamma$ to $\eta\left(\mathrm{m}_{z}\right)$ are $0.4821^{*} \mathrm{~m}_{e}, 0.5317^{*} \mathrm{~m}_{e}$ and $0.5048^{*} \mathrm{~m}_{e}$ respectively. The harmonic mean of the three effective mass values given in Equation 4.4 gives the effective mass of the spin down conduction band to be $\mathrm{m}_{\downarrow}=0.5054^{*} \mathrm{~m}_{e}$, which is in close approximation to the value of $0.52 * \mathrm{~m}_{e}$ reported in the literature [90]. Compared to the $\downarrow$ channel, the $\uparrow$ channel has a lower effective mass leading to its high mobility but as the energy gap of the $\uparrow$ conduction band from the Fermi region being high $1.1689 \mathrm{eV}$ in comparison to $0.3262 \mathrm{eV}$ of the YIG lattice, makes $\uparrow$ channel less conductive. Having the fundamental parameters of YIG, such as the lattice constant, band gap and effective mass match with the literature, a similar first principles approach is implemented for La:YIG. 


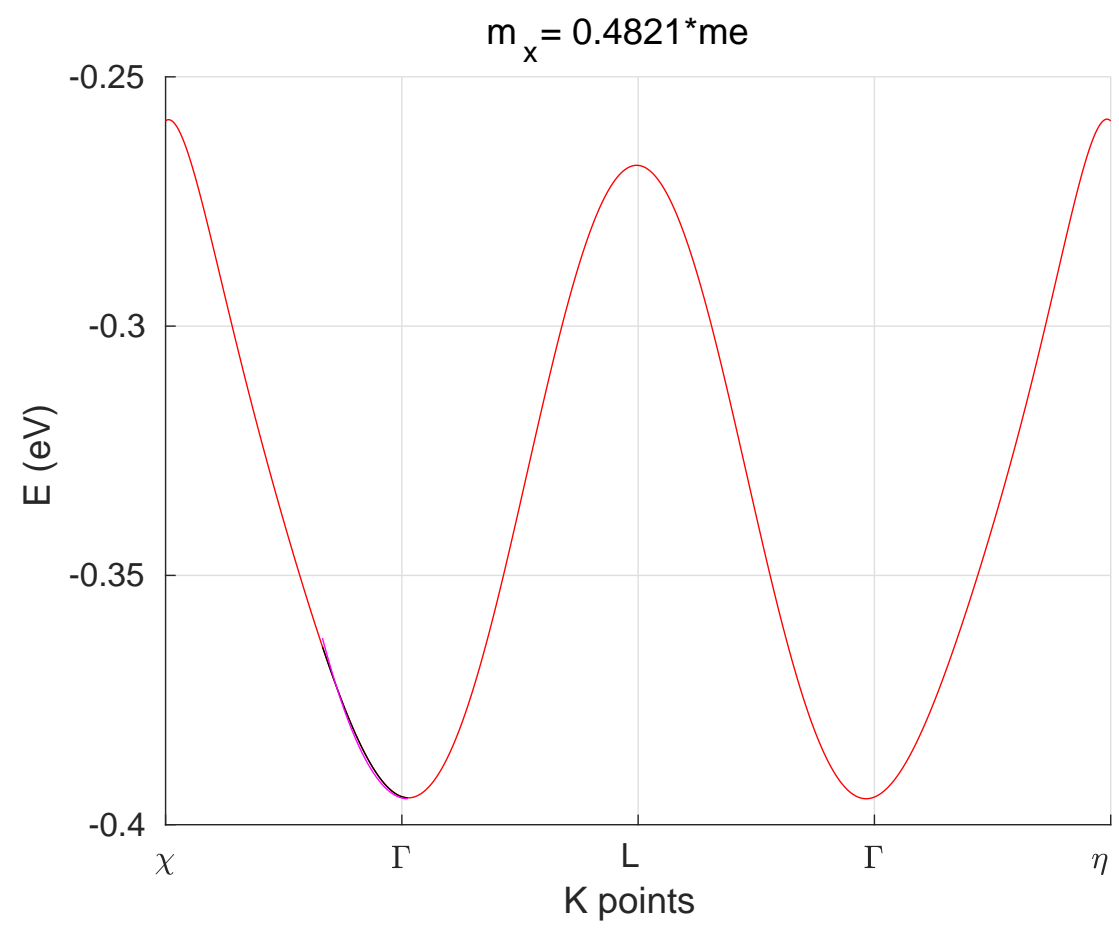

Figure 4.8 Effective mass of spin down conduction band of YIG in X to $\Gamma$ direction, $\left(\mathrm{m}_{x}\right)$. The blue color curve shows the fit of effective mass using the Equation 4.1 with a value of $\mathrm{m}_{x}=0.4821^{*} \mathrm{~m}_{e}$.

$$
\begin{array}{r}
E_{\downarrow(\uparrow)}(k)=E_{\downarrow(\uparrow) c x}+\frac{\hbar^{2} * k_{x-\Gamma}^{2}}{2 * m_{\downarrow(\uparrow) x}^{*}}, \\
E_{\downarrow(\uparrow)}(k)=E_{\downarrow(\uparrow) c y}+\frac{\hbar^{2} * k_{y-\Gamma}^{2}}{2 * m_{\downarrow(\uparrow) y}^{*}}, \\
E_{\downarrow(\uparrow)}(k)=E_{\downarrow(\uparrow) c z}+\frac{\hbar^{2} * k_{z-\Gamma}^{2}}{2 * m_{\downarrow(\uparrow) z}^{*}}, \\
m_{\downarrow(\uparrow) \text { conduction }}^{*}=3 *\left[\frac{1}{m_{\downarrow(\uparrow) x}^{*}}+\frac{1}{m_{\downarrow(\uparrow) y}^{*}}+\frac{1}{m_{\downarrow(\uparrow) z}^{*}}\right]^{-1},
\end{array}
$$

where $\mathrm{k}$ is the wavevector and $\mathrm{E}_{\downarrow(\uparrow) c x}, \mathrm{E}_{\downarrow(\uparrow) c y}$ and $\mathrm{E}_{\downarrow(\uparrow) c z}$ are the conduction band edges in the $\mathrm{x}-\Gamma, \mathrm{y}-\Gamma$ and $\mathrm{z}-\Gamma$ directions respectively of $\downarrow(\uparrow)$ electrons. 


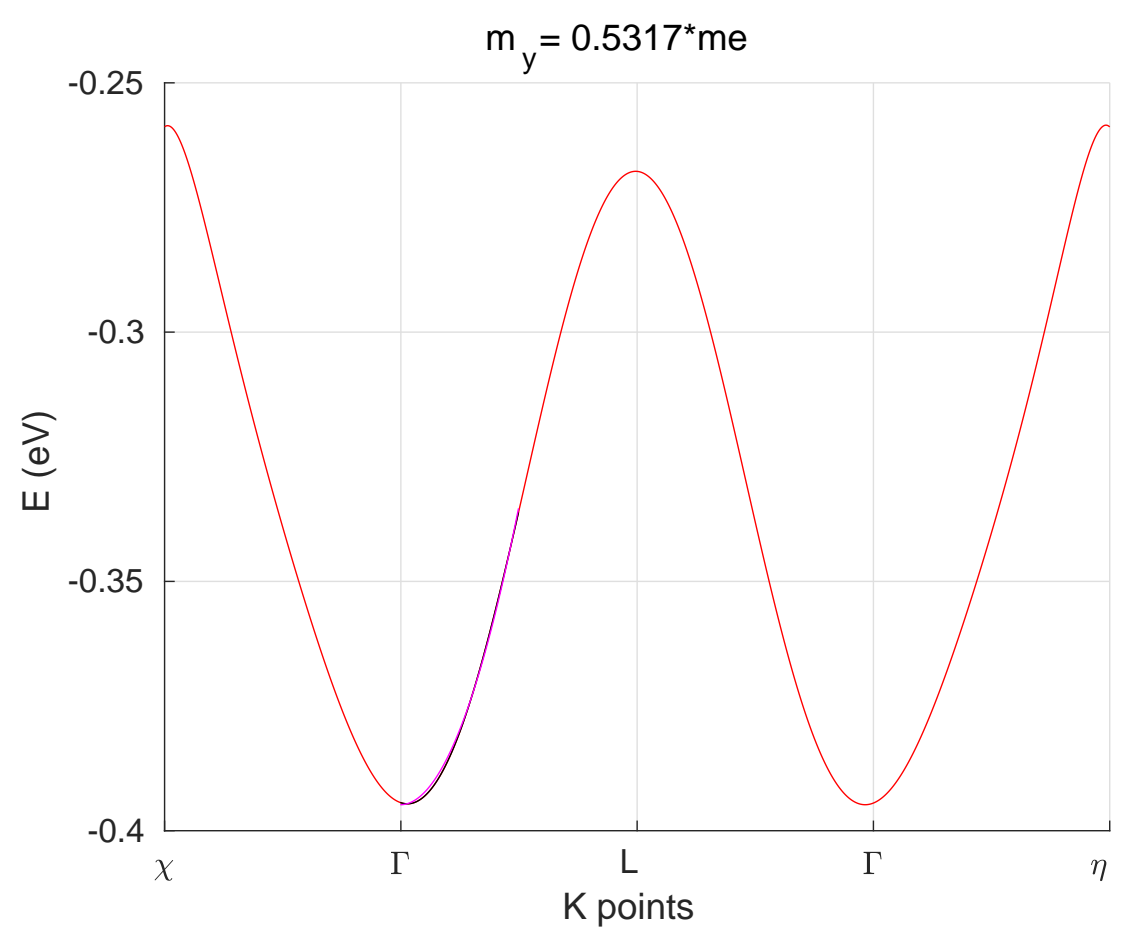

Figure 4.9 Effective mass of spin down conduction band of YIG in $\Gamma$ to $\mathrm{L}$ direction, $\left(\mathrm{m}_{y}\right)$. The blue color curve shows the fit of effective mass using the Equation 4.2 with a value of $\mathrm{m}_{y}=0.5317^{*} \mathrm{~m}_{e}$.

\subsection{Fundamental parameters of La:YIG}

The $\mathrm{Y}$ in the garnets are large cations having dodecahedral coordination. Substituting one $\mathrm{Y}$ atom with another large cation La yields $\mathrm{LaY}_{2} \mathrm{Fe}_{5} \mathrm{O}_{12}$ (La:YIG). Figure 4.11 shows the super cell of La:YIG. Here $4 \mathrm{Y}$ atoms are replaced with 4 La atoms and the relaxation of the unit cell is made following the description given in computational details section. As there are $12 \mathrm{Y}$ positions in an 80-atom super cell, to replace $4 \mathrm{Y}$ atoms with 4 La atoms there are 12C4 combinations. 12C4 yields 495, meaning 495 various unit cells must be optimized and the optimal cell that gives the least energy state must be chosen for further analysis. As this is computationally expensive, one means to perform this task is by mixing the pseudopotentials available in quantum espresso package. Mixing Y and La pseudopotentials can give freedom in choosing positions to replace $\mathrm{Y}$ with La. Following the mixing procedure embedded with quantum espresso package, a convergence in total energy and forces was 


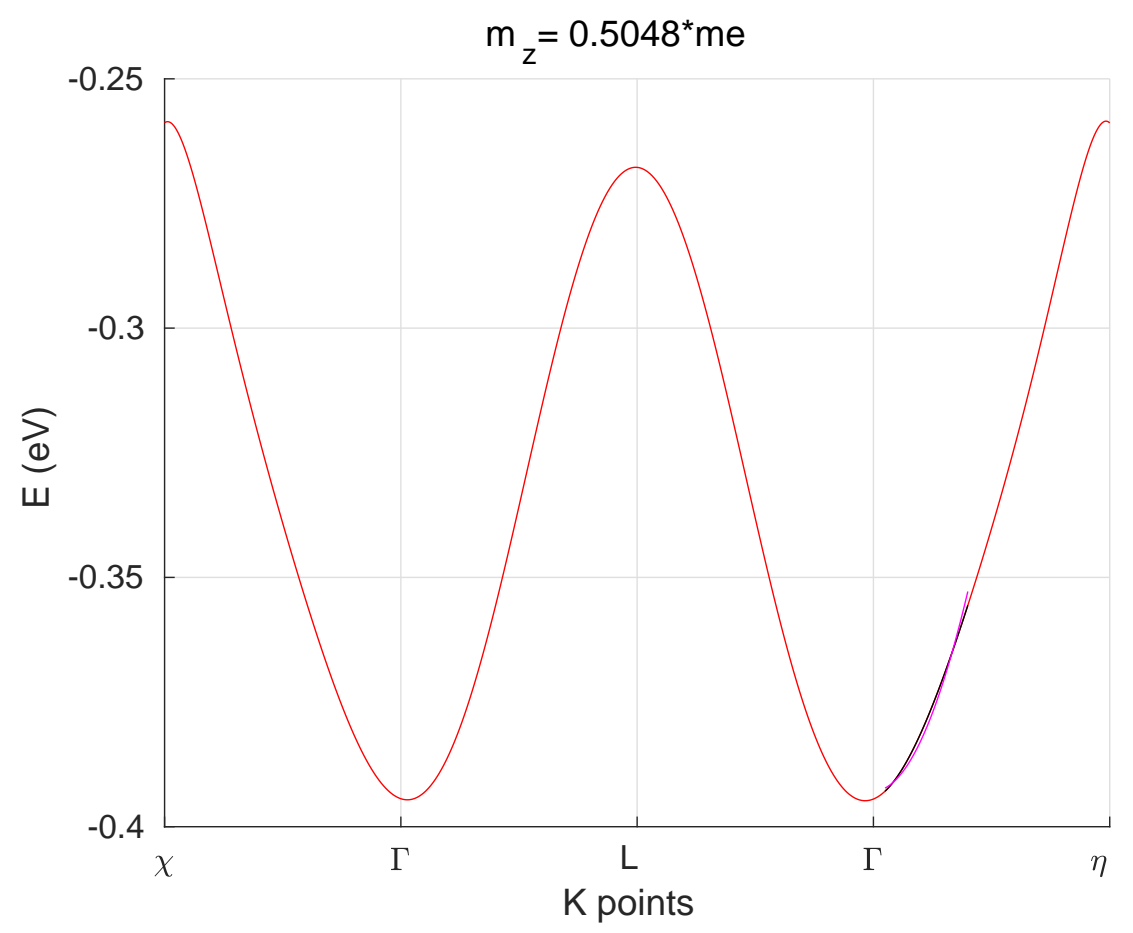

Figure 4.10 Effective mass of spin down conduction band of YIG in $\Gamma$ to $\eta$ direction, $\left(\mathrm{m}_{z}\right)$. The blue color curve shows the fit of effective mass using the Equation 4.3 with a value of $\mathrm{m}_{z}=0.5048^{*} \mathrm{~m}_{e}$.

achieved with the energy cutoff values the same as discussed in computational details section. The relaxed unit cell was used to obtain their electronic band structure, density of states, band gap, electron effective mass, the Fermi energy level, and the magnetization of the material. The lattice parameter increased by $0.87 \%$ compared to pure YIG due to La atoms being larger compared to $\mathrm{Y}$ and acquiring a value of $11.72 \stackrel{\circ}{A}$. These properties were used to calculate its spin transport characteristics in the presence of a temperature gradient.

As shown in Figures 4.12, 4.13 both the $\uparrow$ and $\downarrow$ channels of La:YIG with band gaps of $1.1546 \mathrm{eV}$ (Figure 4.12) and $2.1143 \mathrm{eV}$ (Figure 4.13) respectively, saw a reduction in band gaps when compared with respective counter parts of YIG (Figures 4.5 and 4.6). But the overall band gap of the La:YIG material with $0.3544 \mathrm{eV}$ (Figure 4.14) has a slight increase in the band gap when compared to YIG material (Figure 4.7). This makes each independent 


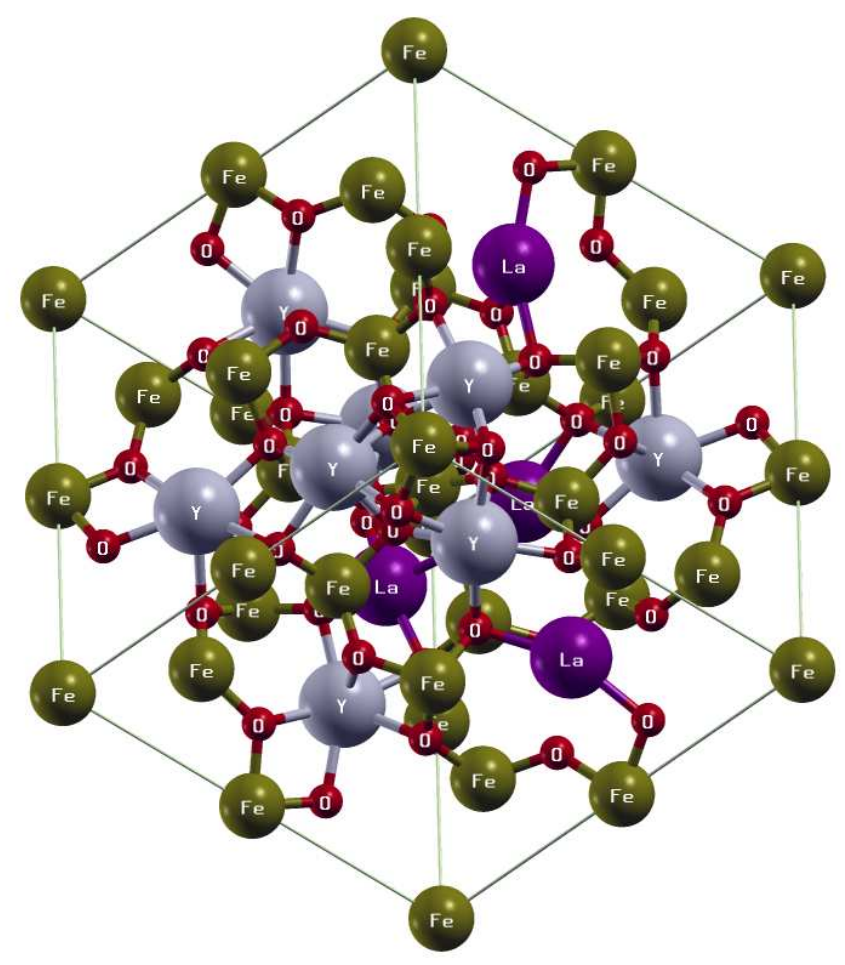

Figure 4.11 A 80 atom super cell of La:YIG. The green atoms represent $\mathrm{Fe}^{+3}$ ions, the gray atoms represent $\mathrm{Y}^{+3}$ ions and the red atoms represent $\mathrm{O}^{-2}$ ions. The four purple atoms in La:YIG super cell that replace the $\mathrm{Y}^{+3}$ ions represent $\mathrm{La}^{+3}$ ions. The positive density of states represent the contribution due to spin up electrons and the negative density of states represent the contribution due to spin down electrons.

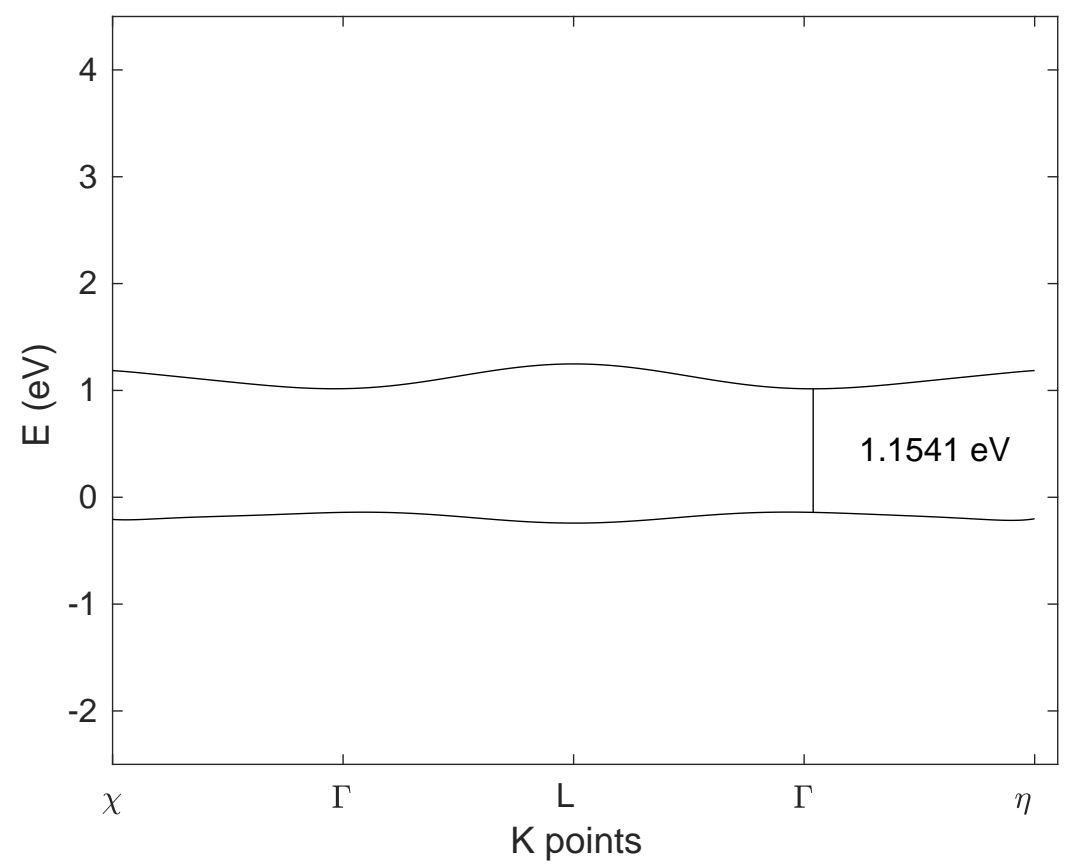

Figure 4.12 The spin up channel of La:YIG material 


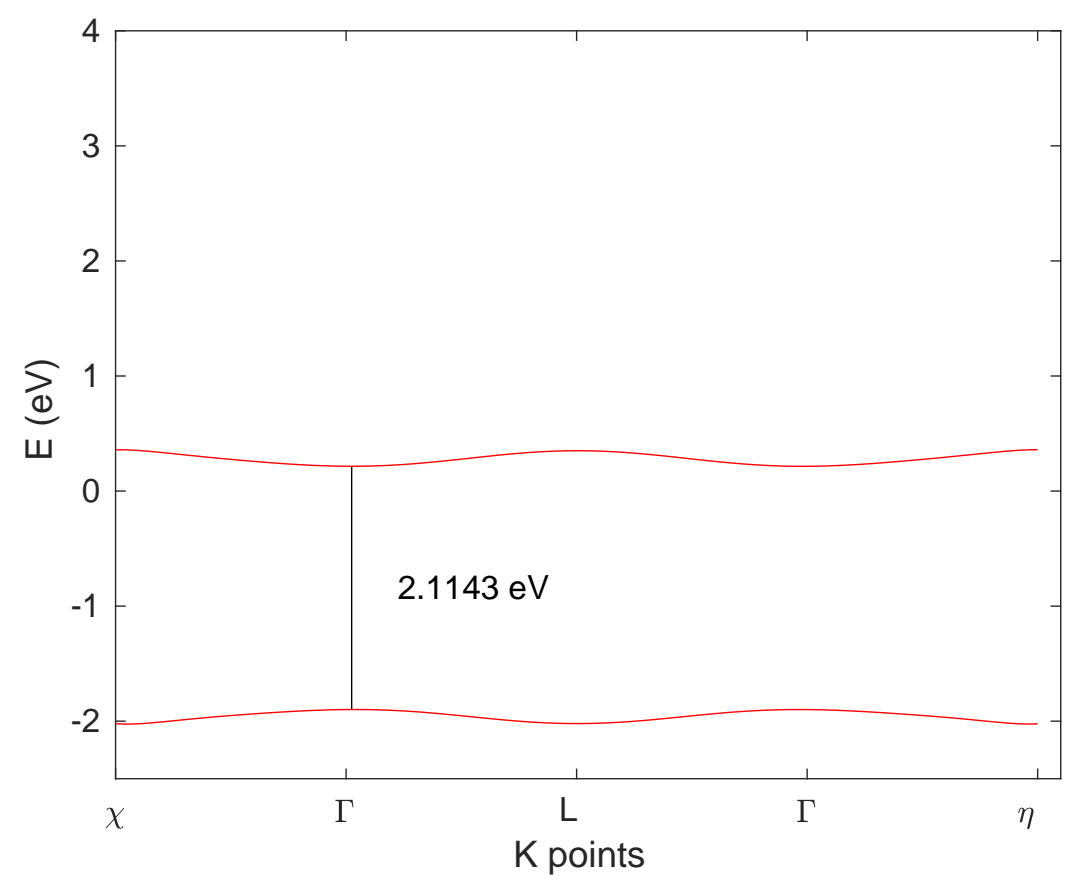

Figure 4.13 The spin down channel of La:YIG material

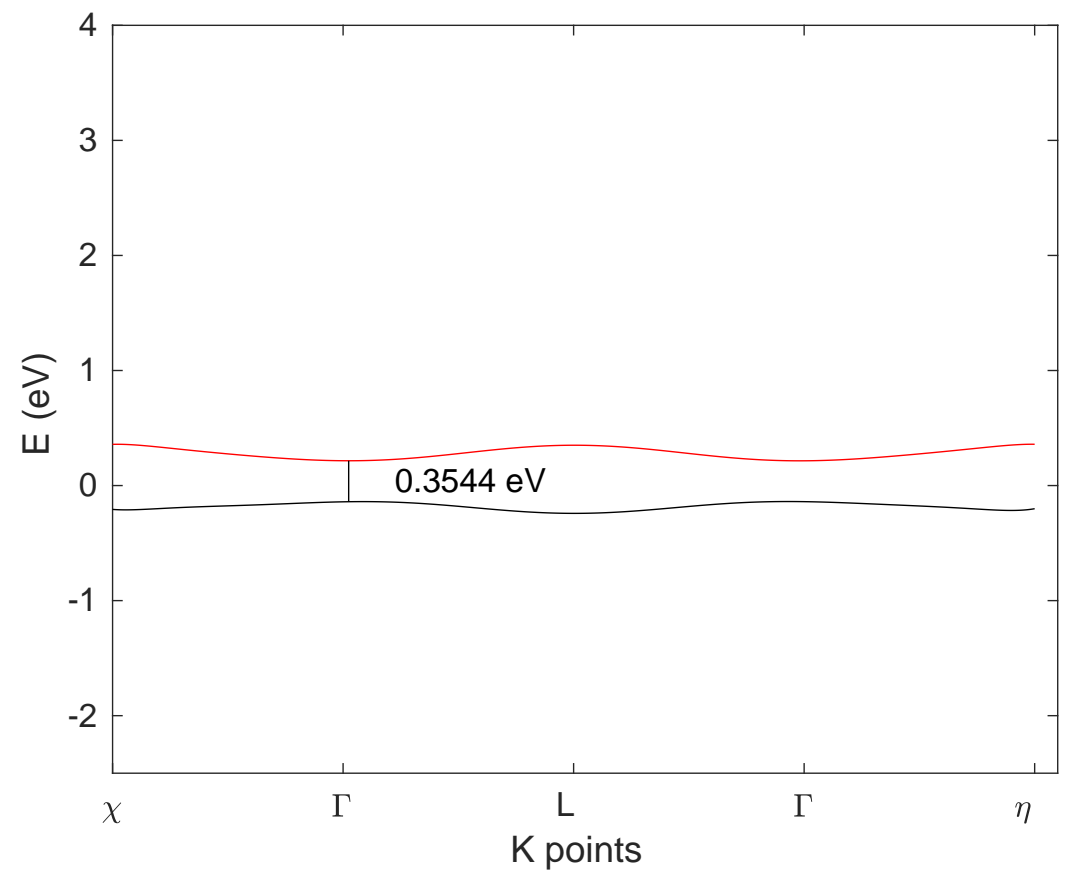

Figure 4.14 The valence band corresponds to the spin up channel and the conduction band corresponds to the spin down channel of La:YIG material. 


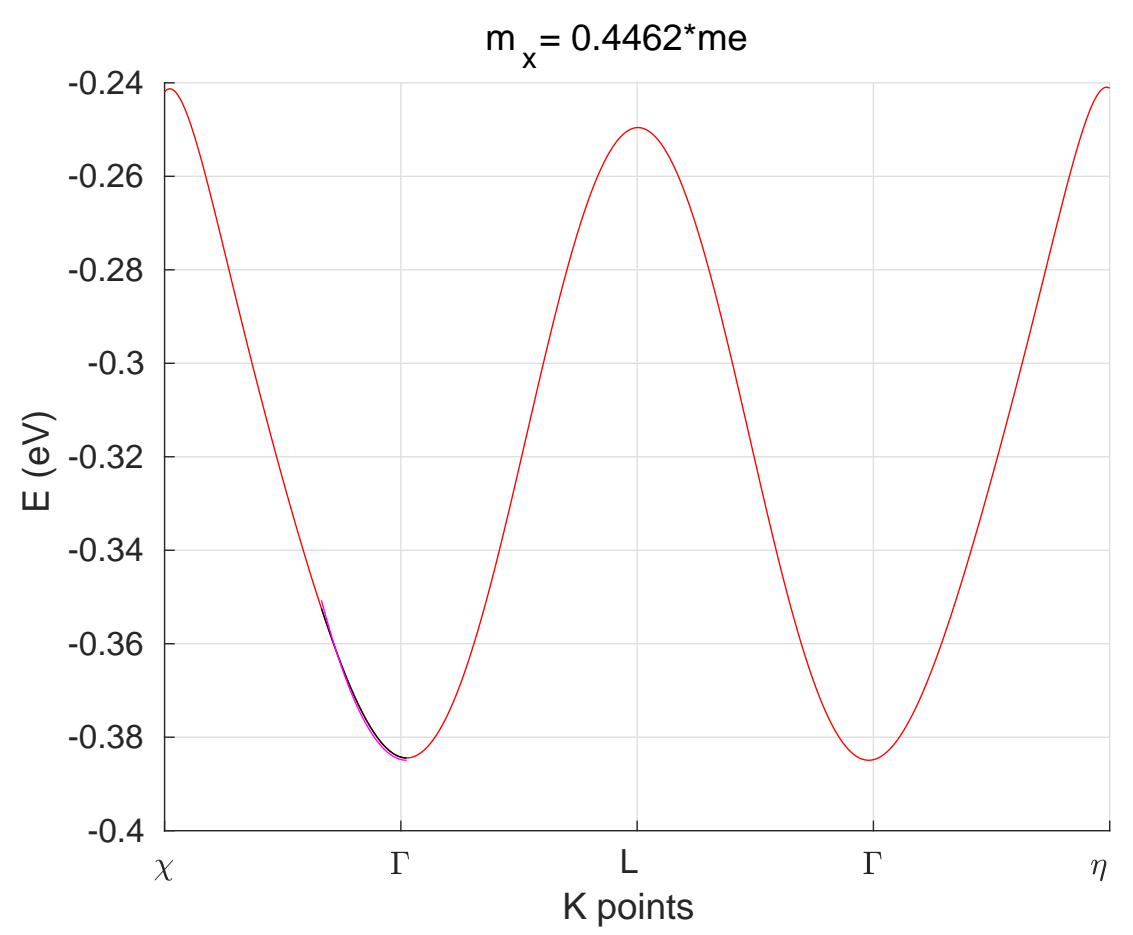

Figure 4.15 Effective mass of spin down conduction band of La:YIG in X to $\Gamma$ direction, $\left(\mathrm{m}_{x}\right)$. The blue color curve shows the fit of effective mass using the Equation 4.1 with a value of $\mathrm{m}_{x}=0.4462^{*} \mathrm{~m}_{e}$.

channel of La:YIG to be more conductive while the material is more insulating than YIG. Like YIG, La:YIG also has spin up channel to contribute to the valence band while spin down channel contributes to the conduction band. The effective mass values of La:YIG in the three reciprocal vector directions $\chi-\Gamma\left(\mathrm{m}_{x}\right)$ shown in Figure 4.15, $\Gamma$ to $\mathrm{L}\left(\mathrm{m}_{y}\right)$ shown in Figure 4.16 and $\Gamma$ to $\eta\left(\mathrm{m}_{z}\right)$ shown in Figure 4.17 are $0.4462^{*} \mathrm{~m}_{e}, 0.4898^{*} \mathrm{~m}_{e}$ and $0.4577^{*} \mathrm{~m}_{e}$ respectively. The harmonic mean of the three the values yields the effective mass of the $\downarrow$ conduction band of La:YIG to be $0.4639^{*} \mathrm{~m}_{e}$. The effective mass of La:YIG is less than that of YIG which has $0.5048^{*} \mathrm{~m}_{e}$, thus making the conduction electrons of La:YIG to have more mobility than YIG.

Figure 4.18 shows the calculated total density of states of La:YIG represented by the formula $\mathrm{LaY}_{2} \mathrm{Fe}_{5} \mathrm{O}_{12}$. The immediate states below Fermi energy level are contributed by spin 


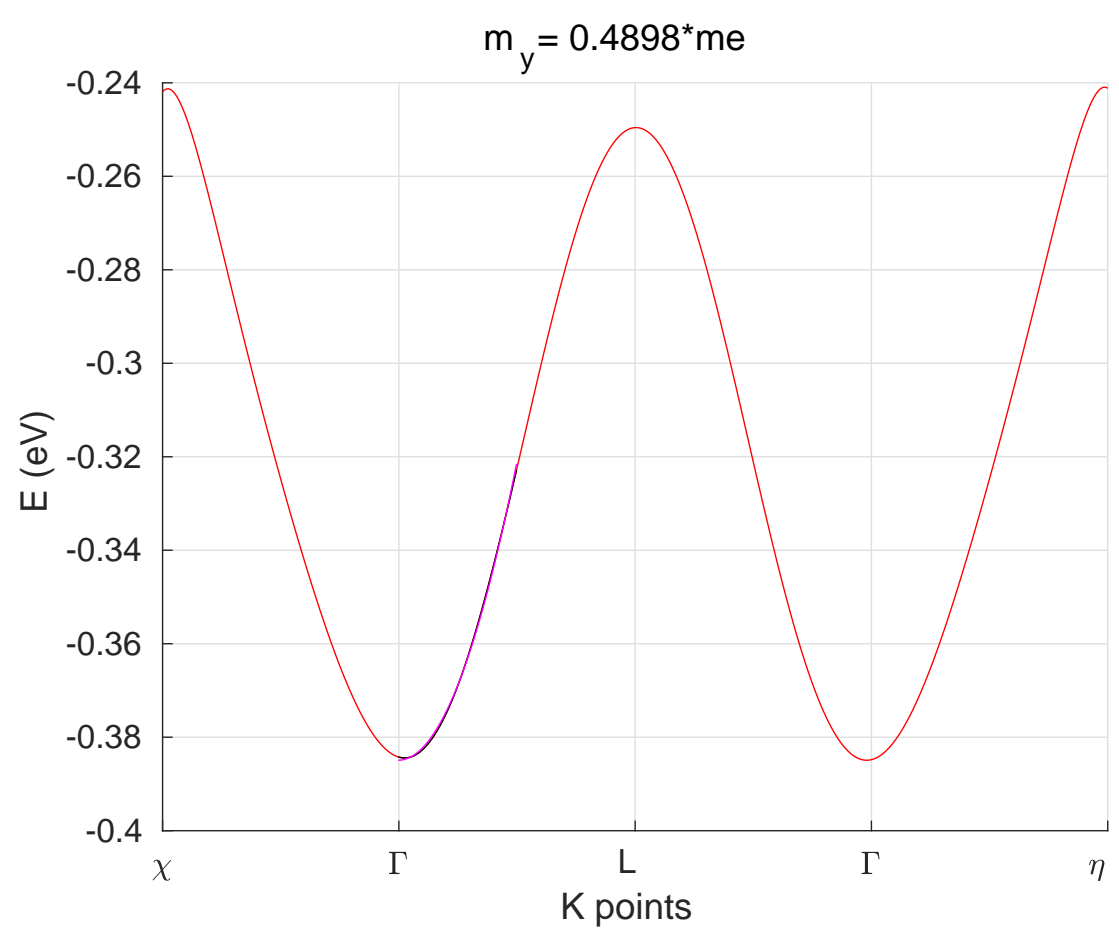

Figure 4.16 Effective mass of spin down conduction band of La:YIG in $\Gamma$ to L direction, $\left(\mathrm{m}_{y}\right)$. The blue color curve shows the fit of effective mass using the Equation 4.2 with a value of $\mathrm{m}_{y}=0.4898^{*} \mathrm{~m}_{e}$.

up states of $\mathrm{O}^{-2}$ and $\mathrm{Fe}^{+3}$ ions and the available states above fermi are due to spin down electrons from $\mathrm{Fe}^{+3}$ ions in the tetrahedral sites similar to that of YIG. Substituting Y with La increased the band gap while decreasing the effective mass of the material. These changes in the fundamental quantities effect the electron and spin transport properties of the material.

\subsection{Validation}

After creating the Hamiltonian of the scattering region, a fine spectrum of energy bands in a range 0 to $5 \mathrm{eV}$ was created for each eigen energy level to calculate the total current in the region due to thermal bias. As the model treats the two spin channels independent, the relative movement of the spin up and spin down electrons is different and leads 


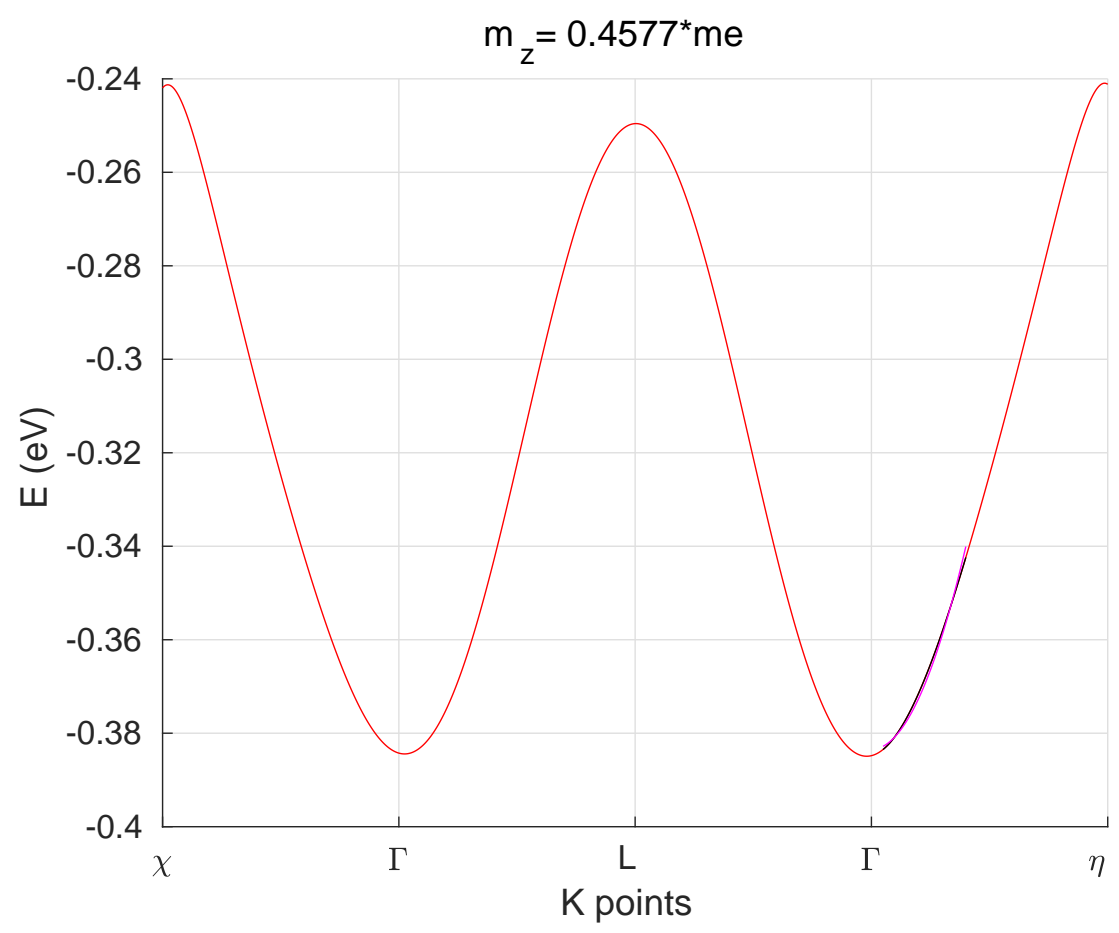

Figure 4.17 Effective mass of spin down conduction band of La:YIG in $\Gamma$ to $\eta$ direction, $\left(\mathrm{m}_{z}\right)$. The blue color curve shows the fit of effective mass using the Equation 4.3 with a value of $\mathrm{m}_{z}=0.4577^{*} \mathrm{~m}_{e}$.

to a spin redistribution in the material due to the non-equilibrium induced by the contacts.

Using the fundamental parameters from DFT calculation and the approach described in the Chapters 2 and 3 of this thesis, was implemented in MATLAB to calculate the spin voltage in the transversely attached Pt NM contact. To compare with the literature, Uchida et al [67], a temperature bias of $20 \mathrm{~K}$ is assumed through the analysis. The calculations were performed at $300 \mathrm{~K}$ temperature (of the NM contact) and linear increase of temperature from left contact (cold) to the right contact (hot). Table 4.1 shows the experimental values and the parameters incorporated in the model. The model used the length of the scattering region along $\mathrm{x}$-direction to be $36 \mathrm{~nm}$. A higher length can be incorporated depending on the computational power in reach. On an 8-core processor, a 36nm length scale took 4 hours to complete the calculation. The number of grid points, $\mathrm{N}=300$, were chosen until the relative 


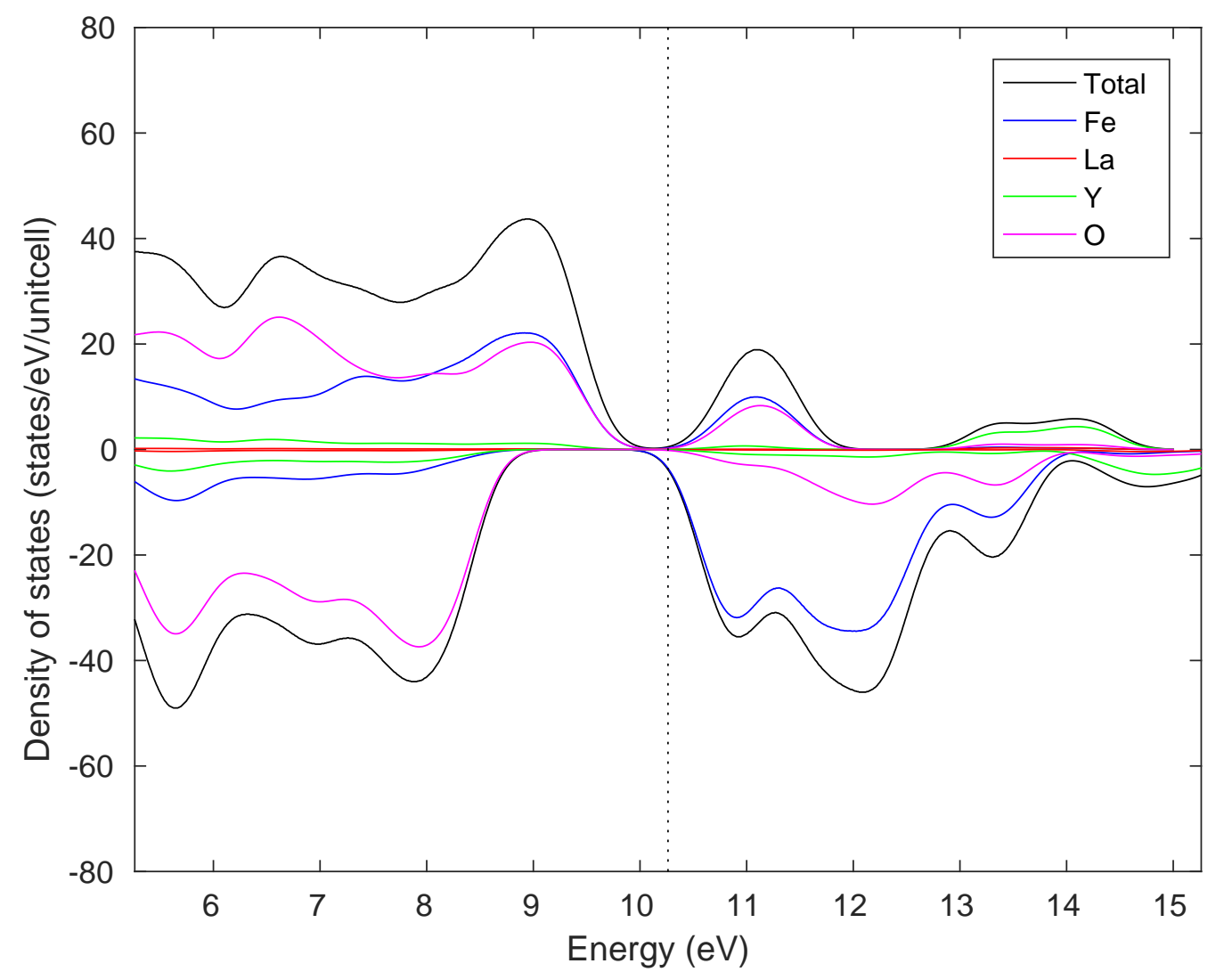

Figure 4.18 The atom projected density of states of iron, lanthanum, yttrium and oxygen show their contributions towards the total density of states near the Fermi region in the La:YIG material lattice.

channel potential $(\mathrm{U})$ reached a value $1 \mathrm{E}-6 \mathrm{eV}$.

The model incorporates the same ratio of length ${ }_{x-\text { direction }}:$ length $_{y-\text { direction }}: \operatorname{length}_{z-\text { direction }}$ of Pt NM contact as used in experimental verification. The ratio being $1: 40: 15 \mathrm{E}-5$. Here, the length of the Pt strip along y direction is related to the length of the La:YIG material along the y-direction which are the same values. Similarly, the length of FM material along the $\mathrm{x}$-direction is related to the length of the NM contact along the y-direction. The ratio of the parameters in the model are same as those in the experimental setup. As a 1-D model was developed in this research, the quantities like spin current injected into the NM contact along the z-direction have the units $\mathrm{A} / \mathrm{m}^{2}$ and the population density of individual spin channels will have the units $1 / \mathrm{m}^{2}$. Hence, the spin populations at each grid point along 
Table 4.1 Parameters used in the experimental setup as reported in literature [67] and the parameters used in verification.

\begin{tabular}{|c|c|c|c|}
\hline Material & $\begin{array}{c}\text { Length } \\
\text { x-direction }\end{array}$ & $\begin{array}{c}\text { Length } \\
\text {-direction }\end{array}$ & $\begin{array}{c}\text { Length } \\
\text { z-direction }\end{array}$ \\
\hline $\begin{array}{c}\text { La:YIG } \\
\text { experimental }\end{array}$ & $8 \mathrm{~mm}$ & $4 \mathrm{~mm}$ & $3.9 \mu \mathrm{m}$ \\
\hline $\begin{array}{c}\text { Pt } \\
\text { experimental }\end{array}$ & $0.1 \mathrm{~mm}$ & $4 \mathrm{~mm}$ & $15 \mathrm{~nm}$ \\
\hline $\begin{array}{c}\text { NEGF model } \\
\text { scattering region }\end{array}$ & $36 \mathrm{~nm}$ & $18 \mathrm{~nm}$ & $17.5 \mathrm{E}-3 \mathrm{~nm}$ \\
\hline $\begin{array}{c}\text { NEGF model } \\
\text { NM contact }\end{array}$ & $0.45 \mathrm{~nm}$ & $18 \mathrm{~nm}$ & $6.75 \mathrm{E}-5 \mathrm{~nm}$ \\
\hline
\end{tabular}

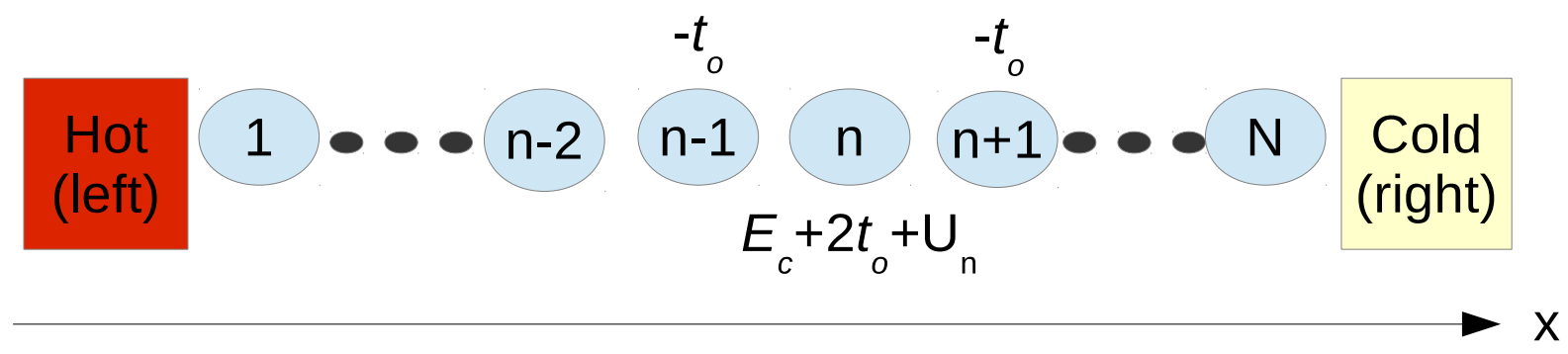

Figure 4.19 Description of the scattering region divided into $\mathrm{N}$ lattice points. The number of grid points $\mathrm{N}$ depends on the length of the lattice.

x-direction, Figure 4.19, were calculated and multiplied with the area of contact between NM (Pt) and FM (La:YIG), which is $0.45 \times 18 \mathrm{~nm}^{2}$. The difference in the populations of the spin channels causes the spin current to flow along the z-direction into the transversely NM contact. This spin current from La:YIG is converted into inverse spin Hall voltage $\mathrm{V}_{I S H E}$ in the Pt contact. The other constants extracted from literature are shown in Table 4.2.

Incorporating the parameters from Tables 4.1, 4.2 in the aforementioned model, the 
Table 4.2 Constants incorporated in the model.

\begin{tabular}{|c|c|}
\hline Constant & Value \\
\hline $\begin{array}{c}\gamma \\
\text { reference }([85])\end{array}$ & $1.4^{*} 10^{11} 1 / \mathrm{T} . \mathrm{s}$ \\
\hline $\begin{array}{c}\rho \\
\text { reference }([89])\end{array}$ \\
electrical resistivity of Pt at 300K \\
\hline $\begin{array}{c}\mathrm{g}_{r} / \mathrm{A} \\
\text { reference }([104])\end{array}$ & $0.1^{*} 10^{16} 1 / \mathrm{m}^{2}$ \\
\hline $\begin{array}{c}\theta_{H} \\
\text { reference }([105]) \\
\text { Hall angle of Pt }\end{array}$ & 0.0037 \\
\hline $\begin{array}{c}\mathrm{V}_{a} \\
\text { volume of } \mathrm{NM}\end{array}$ & $0.45^{*} 18^{*} 6.75 \mathrm{E}-5 \mathrm{~nm}^{3}$ \\
\hline
\end{tabular}

$\mathrm{V}_{I S H E}$ in the transversely attached Pt electrode at various locations along the scattering region is shown in Figure 4.20. The red line represents the trend calculated from the NEGF model for a $20 \mathrm{~K}$ temperature bias and the black line with the data points represented in black circles is the experimental trend for the same temperature obtained from literature [67]. The slope of the NEGF trend is close to the experimental data with an error of $6.5 \%$. The developed model can be applied to magnetic materials which have atoms with d-electrons. By assuming a $\pm 5 \%$ error in effective mass, the model predicts the curve with $\pm 15 \%$ close to that of experimental value. Hence, this model can be applicable to materials whose experimental transverse spin properties are not available.

With the given thermal difference between contacts the charge carriers drift away from the hot source and towards the cold source. This drift rate which related to the electric conductivity is different for the two spin channels. Hence, the presence of heat redistributes the spin carriers and thus causes a net difference in their populations along the material lattice. It has been experimentally verified that this difference in the spin populations is 


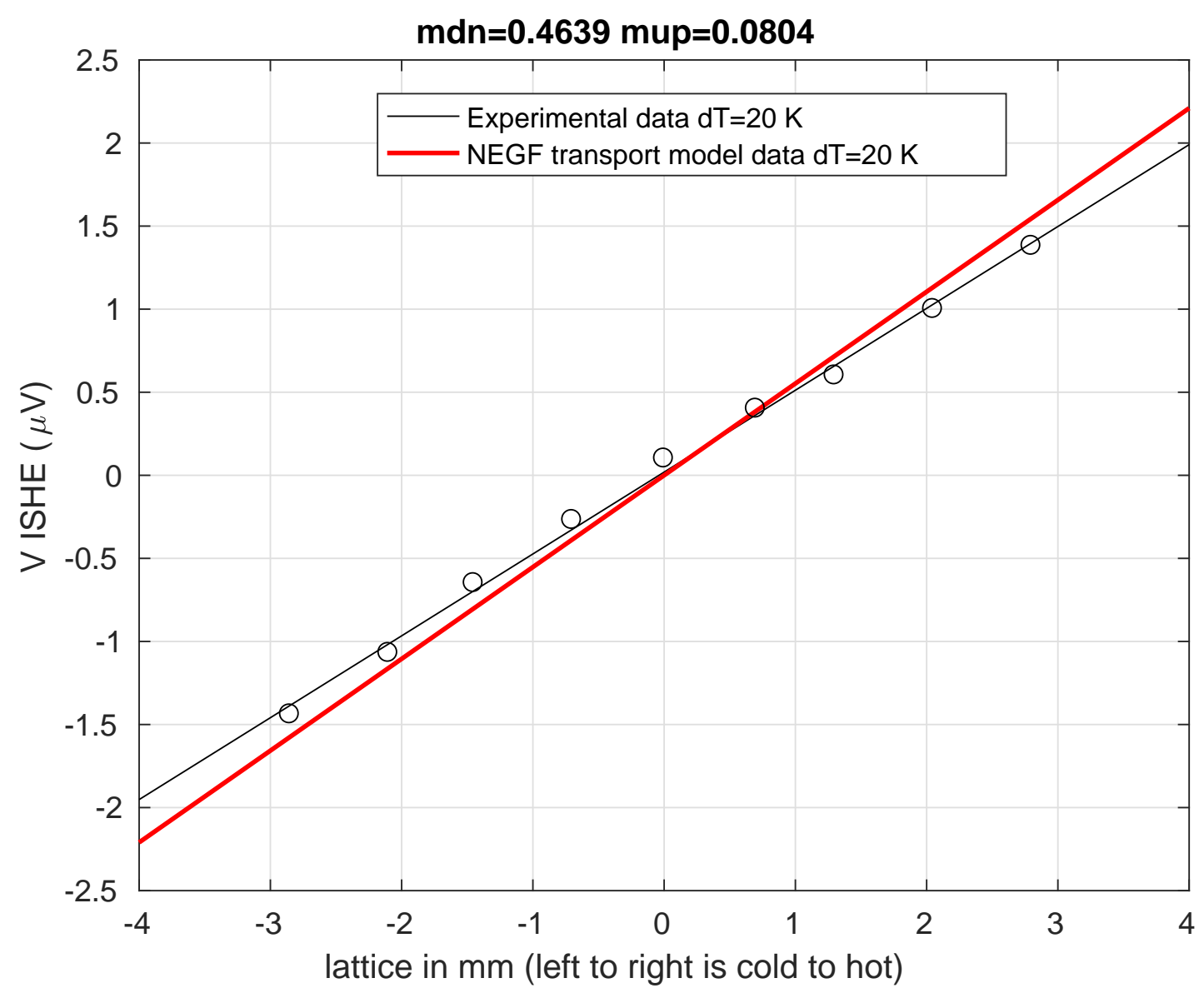

Figure 4.20 Validation of the model with the experimental data available for La:YIG. The $V_{I S H E}$ voltage in $\mu \mathrm{V}$ is calculated at the different points on the material lattice along the $\mathrm{x}$-direction.

high at the ends of the ferromagnetic material. The same phenomena was observed using the spin transport theory where the Figure 4.20 shows a linear trend calculated from the incorporated theory (represented in red color). This slope is in close approximation to the experimental data available (represented in black color). Interestingly both the experimental data and the NEGF spin transport theory find that the voltage sign changes at the centre of the device proving the uniform spin redistribution in the material due to the temperature 
difference. 


\section{CHAPTER 5}

\section{EXPLORING DESIGN SPACE OF NI AND ZN SUBSTITUTED CO}

\subsection{The effect of band gap and effective mass on Seebeck coefficient}

Performing validation highlights the role of band gap of the material and the effective mass of conduction electrons on the Seebeck coefficient of the material. As DFT is known for its property of underestimating the band gap of materials, sole dependence on the parameters attained from DFT calculations might not always yield accurate data. DFT, on the other hand, can be used to provide a trend to study the effect of substitutions in a material.

As in conventional thermoelectric materials, the electronic and thermal conductivities are intertwined, it is reasonable to think if band gap and effective mass are intertwined. To study this, a Hubbard U parameter is used to increase the band gap and depict the effect of increasing band gap on the shape of the conduction band.

Table 5.1 gives the values of effective mass with an increasing Hubbard U parameter.

With increasing $\mathrm{U}$, the bang gap increased while effective mass showed a decreasing trend.

Table 5.1 The effect of Hubbard U parameter on the band gap and effective mass spin down conduction electrons of La:YIG material.

\begin{tabular}{|c|c|c|c|c|c|c|c|}
\hline \multicolumn{7}{|c|}{ cell parameter, band gap and spin polarization } \\
\hline DFT+U eV & \multicolumn{3}{|c|}{ Effective mass $\mathrm{m}^{*}$} & \multicolumn{3}{c|}{ Band gap in eV } \\
\hline & $\mathrm{m}_{x}^{*}$ & $\mathrm{~m}_{y}^{*}$ & $\mathrm{~m}_{z}^{*}$ & total & spin-up & spin-down & net \\
\hline 0.0 & 0.4462 & 0.4898 & 0.4577 & 0.4639 & 1.1541 & 2.1138 & 0.3539 \\
\hline 3.0 & 0.3478 & 0.5505 & 0.5297 & 0.456 & 3.033 & 2.3912 & 0.4096 \\
\hline 4.0 & 0.29342 & 0.3736 & 0.3739 & 0.3425 & 3.1155 & 2.422 & 0.5786 \\
\hline 5.0 & 0.2516 & 0.3654 & 0.3185 & 0.3046 & 3.1672 & 2.4473 & 0.7016 \\
\hline 6.0 & 0.2386 & 0.31706 & 0.28883 & 0.2776 & 3.2168 & 2.445 & 0.80095 \\
\hline
\end{tabular}


Not only in the overall effective mass, but even along the individual reciprocal vector directions, there is a consistent decrease in the effective mass. Low effective mass makes the electrons to have more mobility while high band gap increases the resistivity.

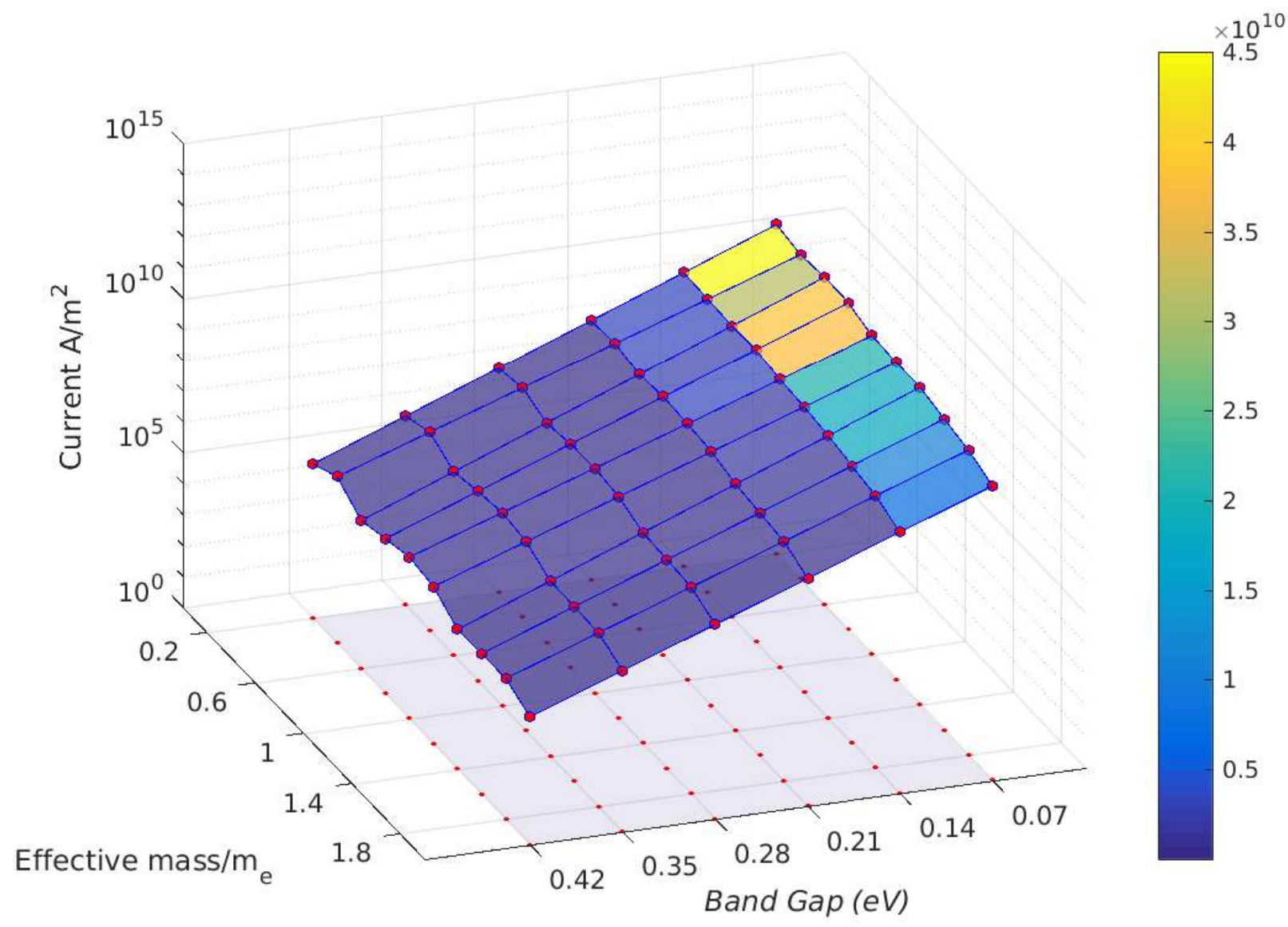

Figure 5.1 The variation of current density generated in the scattering channel with band gap and effective mass of conduction electrons in the scattering channel. The temperature difference between the end contacts was $20 \mathrm{~K}$. The $\mathrm{x}$-axis represents the band gap in the material, y-axis represents $\frac{\text { effectivemass }}{\text { massofelectron }}$ and z-axis represents the current density in the scattering channel on a log scale. An increase in the band gap causes an exponential decrease in the current density. An increase in effective mass causes a linear decrease in the cirrent density.

Using the model developed in this research, the variation of band gap and effective 
mass on the thermoelectric properties was studied. Figure 5.1 shows the variation of the current generated in the material with band gap and effective mass of conduction electrons. The maximum current was attained in materials with low band gap. Low band gap materials have can easily drive the valence electrons to the conduction band and hence are capable of generating high current. In the Figure 5.1, for a band gap of $0.07 \mathrm{eV}$ and low effective mass, a current density of $4^{*} 10^{10} \mathrm{~A} / \mathrm{m}^{2}$ was achieved, while for high band gaps like $0.42 \mathrm{eV} 5^{*} 10^{5}$ $\mathrm{A} / \mathrm{m}^{2}$ was achieved, which is very low compared to $0.07 \mathrm{eV}$ band gap materials.

Effective mass also has an effect on the current generated in the materials. Increasing effective mass decreases the current density generated in the material. As the mobility of electrons inversely varies with the effective mass, lower effective mass causes high electron mobility while materials with high conduction band effective mass have low electron mobility. The z-axis in Figure 5.1 is a $\log$ scale with base 10. An exponential decrease in the electrical conductivity was observed with the increasing band gap, while a linear trend was observed with the variation in effective mass. This suggests that the model is highly sensitive to the band gap of the material than on effective mass of its conduction electrons. Hence, for accurately calculating the current density of a material, an accurate band gap must be known.

Figure 5.2 shows the variation of electrical conductivity in a material with band gap and effective mass of conduction electrons. The maximum electrical conductivity can be attained in materials with low band gap, a similar trend to that of current density in Figure 5.1. Increasing effective mass also decreases the electrical conductivity of a material. The mobility of electrons inversely varies with the effective mass. The z-axis in Figure 5.2 is a log scale with base 10. An exponential decrease in the electrical conductivity was observed with the increasing band gap. Both the conductivity and the current generated in the material can be related to one another as one property effects the other hence, both the current density and the electrical conductivity in the material were effected in a similar manner with 


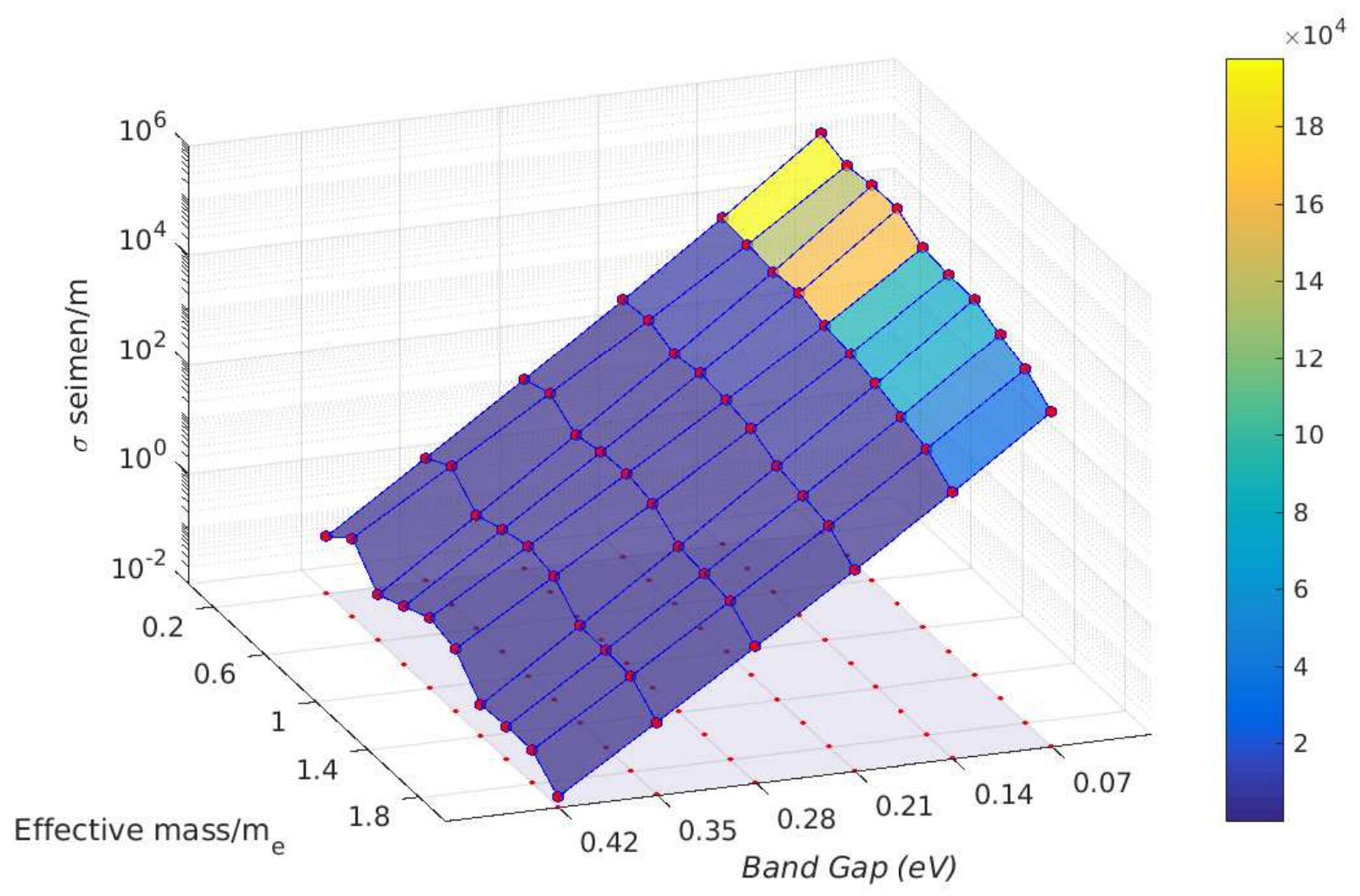

Figure 5.2 The variation of electrical conductivity $(\sigma,($ siemen $/ \mathrm{m}))$ in the scattering region with band gap and effective mass of conduction electrons in the scattering channel. The temperature difference between the end contacts was $20 \mathrm{~K}$. The $\mathrm{x}$-axis represents the band gap in the material, y-axis represents $\frac{\text { effectivemass }}{\text { massofelectron }}$ and z-axis represents the electrical conductivity in a log scale. $\sigma$ decreases exponentially with an increasing band gap.

the variation in the band gaps abd effective mass of conduction electrons.

Figure 5.3, shows the variation of Seebeck coefficient of the material with the band gap and effective mass. The effective mass has a minimal effect on the Seebeck coefficient when compared with the effect caused due to the band gap of the material. Higher the band gap of the material, higher is the Seebeck coefficient. But increasing band gap takes a negative toll on the electrical conductivity as was seed in Figure 5.2. The conductivity decreases by an order of 10 with every $0.07 \mathrm{eV}$ increase in the band gap, while the Seebeck coefficient increases by a magniture of $175 \mu \mathrm{V} / \mathrm{K}$ with every $0.07 \mathrm{eV}$ band gap. 


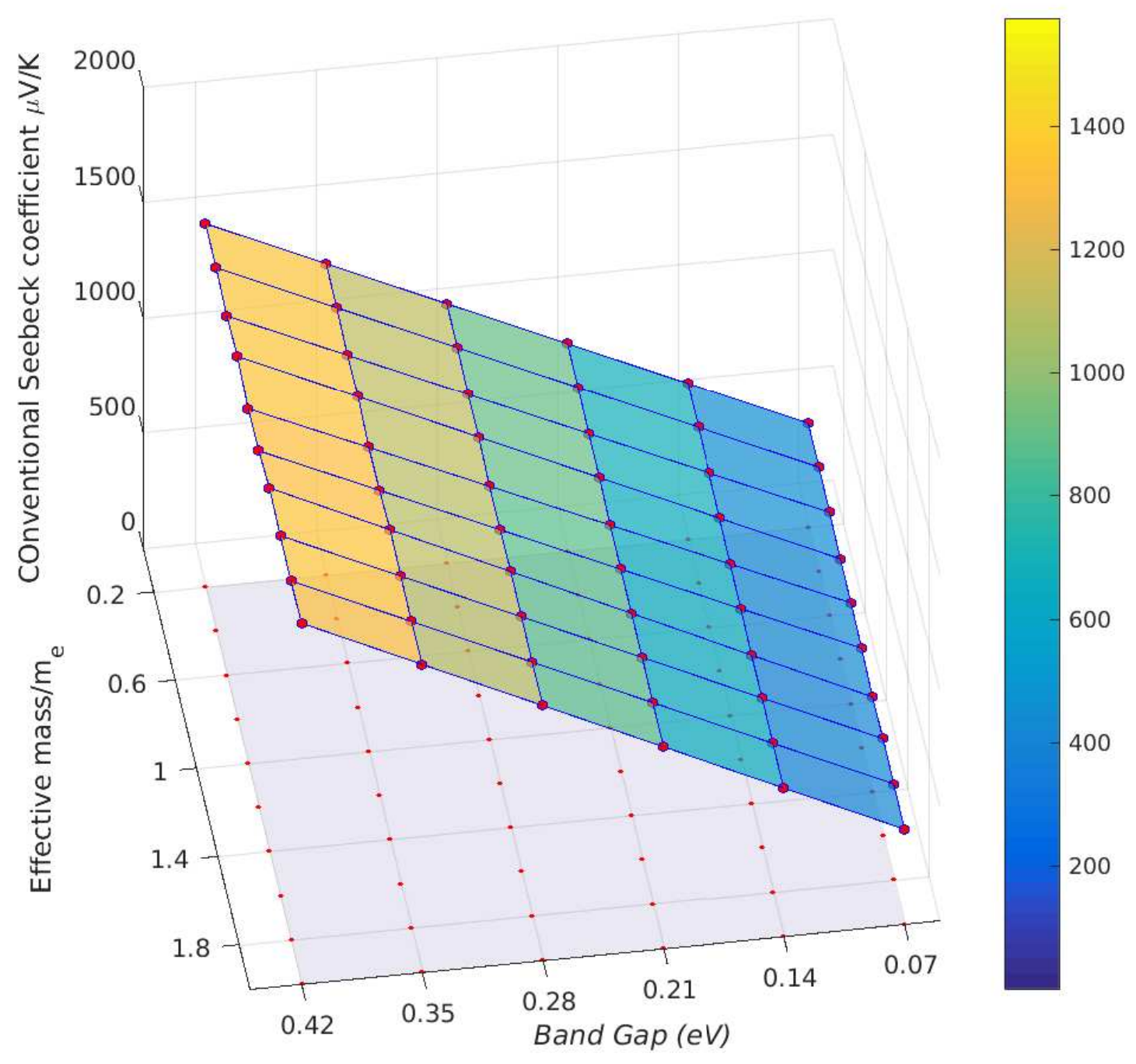

Figure 5.3 The variation of conventional Seebeck coefficient, in $\mu \mathrm{V} / \mathrm{K}$, with band gap and effective mass of conduction electrons in the scattering channel. The temperature difference between the end contacts was $20 \mathrm{~K}$. The $\mathrm{x}$-axis represents the band gap in the material, $\mathrm{y}$-axis represents $\frac{\text { effectivemass }}{\text { massofelectron }}$ and z-axis represents the conventional Seebeck coefficient. Higher band gap materials have high Seebeck coefficient.

In all the above trends, the model developed in this research was highly sensitive to the band gap of the material. And in order to accurately calculate the individual properties, the correct band gap must be obtained from the first principle calculations by incorporating 
a Hubbard U parameter. But the current thesis focuses on the variation of thermoelectric properties with $\mathrm{Zn}$ or $\mathrm{Ni}$ substitution in the $\mathrm{Co}_{3} \mathrm{O}_{4}$ spinel material lattice. Even without using $\mathrm{U}$ paramter, a reliable trend of band gaps with an increasing substitution of another atom in the material lattice can be obtained. Hence, in this research, a U parameter was not introduced to the $\mathrm{Co}_{3} \mathrm{O}_{4}$ spinel lattice. On the other hand, to study the impact of the substitutions in the material, only the trend caused due to substitutions can provide enough detail on tuning the properties. Hence, in this thesis the fundamental parameters of $\mathrm{Co}_{3} \mathrm{O}_{4}$ based spinel oxide materials were calculated without incorporating the Hubbard U paramerter.

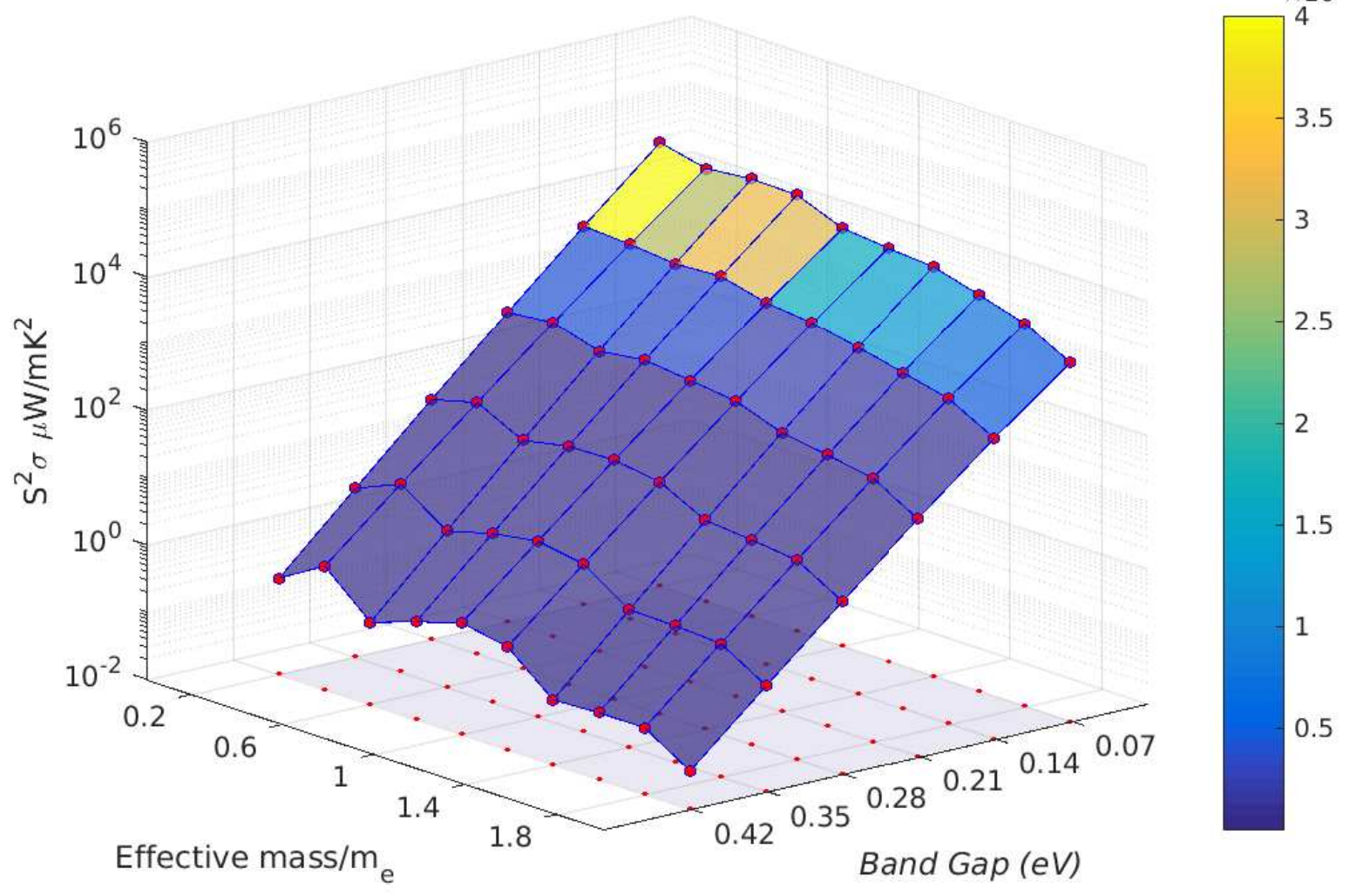

Figure 5.4 The variation of power factor, in $\mu \mathrm{W} / \mathrm{mK}^{2}$, with band gap and effective mass of conduction electrons in the scattering channel. The temperature difference between the end contacts was $20 \mathrm{~K}$. The $\mathrm{x}$-axis represents the band gap in the material, $\mathrm{y}$-axis represents $\frac{\text { effectivemass }}{\text { massofelectron }}$ and $\mathrm{z}$-axis represents the power factor of the material in log scale. 


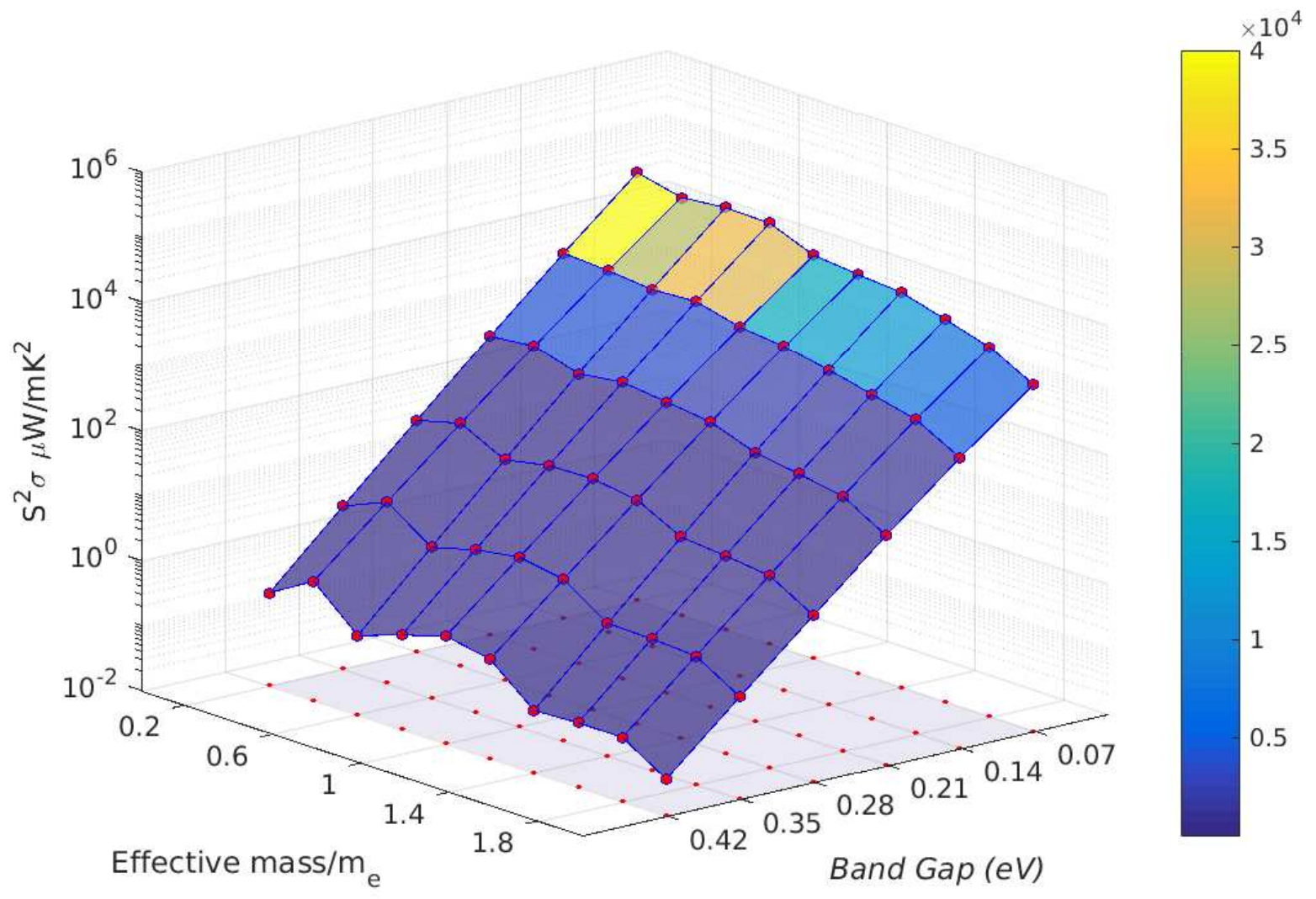

Figure 5.5 The variation of power factor, in $\mu \mathrm{W} / \mathrm{mK}^{2}$, with band gap and effective mass of conduction electrons in the scattering channel. The temperature difference between the end contacts was $20 \mathrm{~K}$. The $\mathrm{x}$-axis represents the band gap in the material, $\mathrm{y}$-axis represents $\frac{\text { effectivemass }}{\text { massofelectron }}$ and $\mathrm{z}$-axis represents the power factor of the material in log scale.

Figure 5.5, shows the variation of power factor of materials with varying band gaps and effective mass of conduction electrons. The power factor of a material is a major contributing factor to improve the thermoelectric performance of a material. Higher the value of power factor, the better is the performance. Power factor is the product of the square of Seebeck coefficient and the electrical conductivity of the material, $\mathrm{S}^{2 *} \sigma$. As shown in Figure 5.5, materials with low band gap seem to have high power factor as their electrical conductivity is huge, given that the high rate of conduction electrons flow is possible in the presence of a thermal gradient. With the Seebeck coefficient being relatively constant with the increasing effective mass compared to electrical conductivity which decreases with then 
increasing $\mathrm{m}^{*}$, a decrease in the overall power factor was observed with increasing band gap.

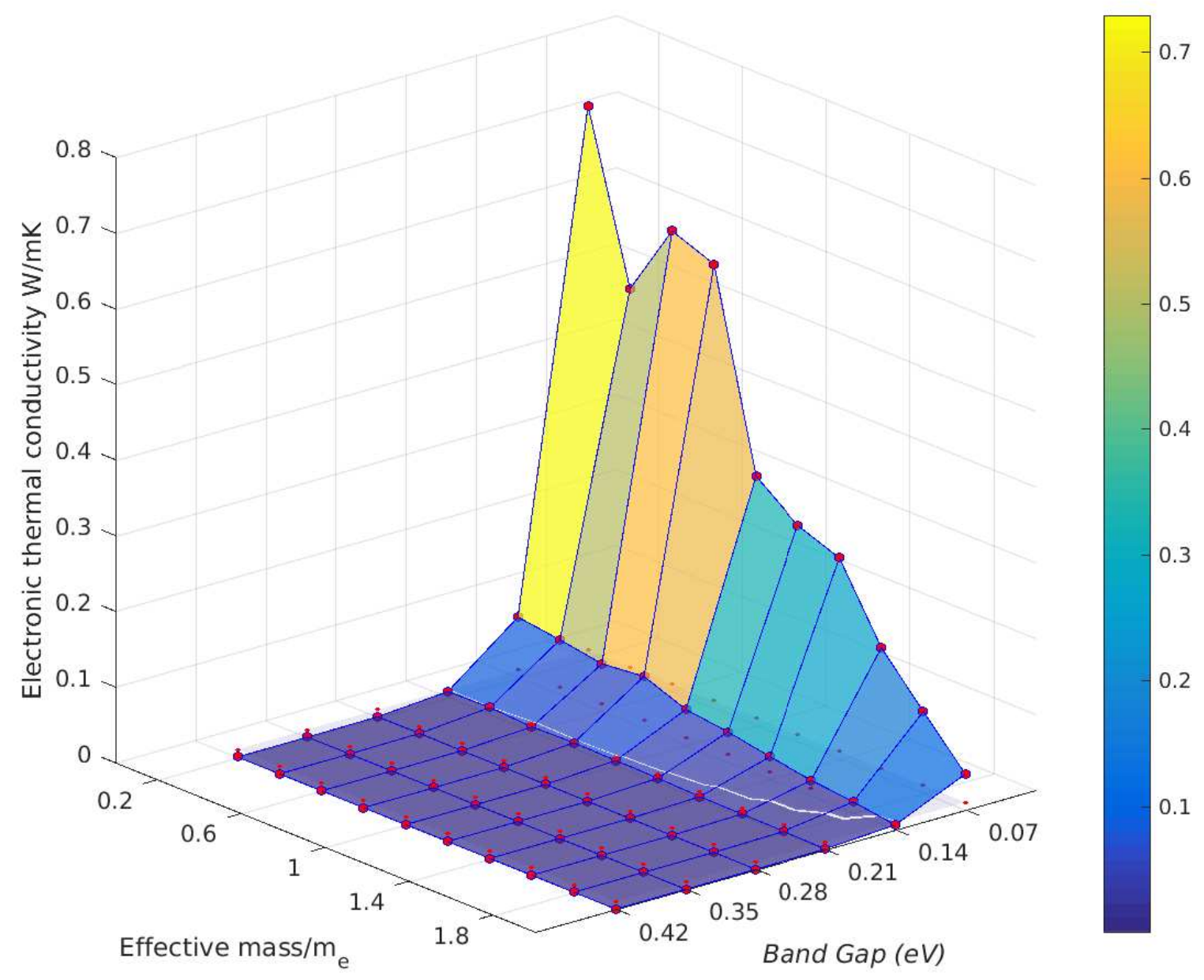

Figure 5.6 The variation of electronic contribution of thermal conductivity, $\kappa_{e}$ in $\mathrm{W} / \mathrm{mK}$, with variation in band gap and effective mass of conduction electrons in the scattering channel. The temperature difference between the end contacts was 20K. The $\mathrm{x}$-axis represents the band gap in the material, y-axis represents $\frac{\text { effectivemass }}{\text { massofelectron }}$ and z-axis represents the $\kappa_{e}$ of the material. The low band gap materials have high electronic contribution towards the thermal conductivity compmared to the low band gap materials.

Figure 5.6, shows the variation of electronic contribution to thermal conductivity of 
materials $\left(\kappa_{e}\right)$ with varying band gaps and effective mass of conduction electrons. The $\kappa_{e}$ of a material inversely effects the performance of a thermoelectric material. Through out the research community, there is a search for low thermlly conductive materials that simoultaneously equip high electrical conductivity. Higher value of $\kappa_{e}$ was found in materials that have high electrical conductiviy as expected. As shown in Figure 5.6, materials with low band gap have high $\kappa_{e}$, as their electrical conductivity is huge, while high band hap materials have relatively lower electronic thermal conductivites.

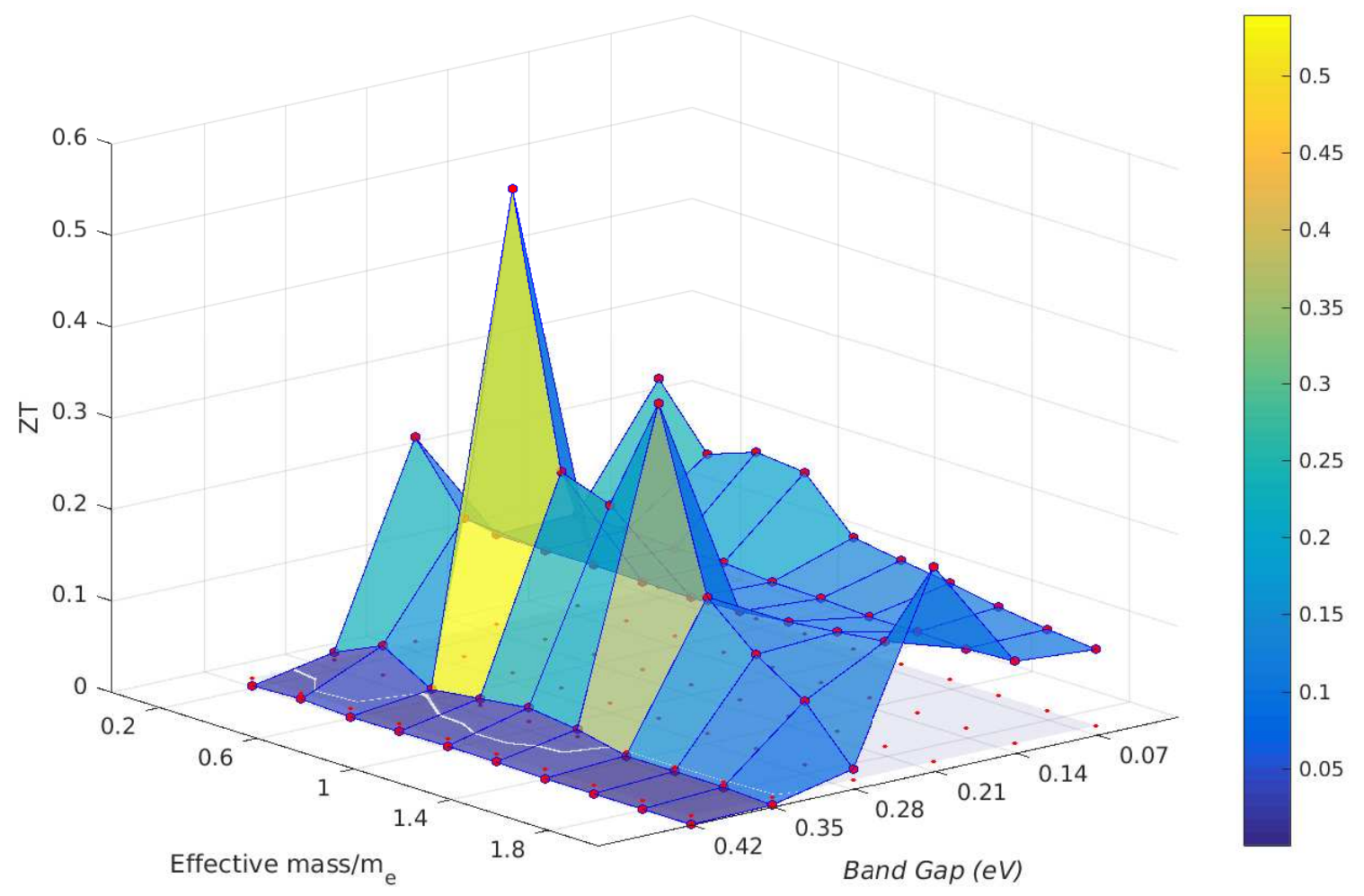

Figure 5.7 The variation of thermoelectric performance, ZT, with variation in band gap and effective mass of conduction electrons in the scattering channel. The temperature difference between the end contacts was $20 \mathrm{~K}$. The $\mathrm{x}$-axis represents the band gap in the material, $\mathrm{y}$ axis represents $\frac{\text { effectivemass }}{\text { massofelectron }}$ and z-axis represents the $\kappa_{e}$ of the material. The intermediate region having the band gaps around $0.14 \mathrm{eV}$ to $0.35 \mathrm{eV}$ seem to posess a ZT with a value of 0.5. Low band gap materials have high electronic thermal conductivity took a toll on their thermoelectric performance. 
The ZT, thermoelectric performance of a material, is an important fator to the thermoelectric research community that provides an estimate of a material's capability in thermoelectric energy applications. The ZT of a material is the ratio of it power factor to it

total thermal conductivity, $\mathrm{ZT}=\frac{S^{2} \sigma}{\kappa_{e}+\kappa_{e}}$. The experimental values of $\kappa_{p}$ of various materials were extracted from literature and passed into the model along with the calculated Seebeck, coefficient, electrical conductivity and electronic thermal conductivity. The Figure 5.7 shows the effect of variation of band gaps and effective mass on a material. It can be observed that an intermediate region having the band gaps around $0.14 \mathrm{eV}$ to $0.35 \mathrm{eV}$ seem to posess a ZT with a value of 0.5 . Also, low effective mass of conduction electrons have a posivite effect on ZT. A value of 0.5 has been calculated for materials with band gap $0.28 \mathrm{eV}$ and an effective mass $0.6^{*} \mathrm{~m}_{e}^{*}$, while a nmaterial with $0.28 \mathrm{eV}$ and an effective mass $1.2^{*} \mathrm{~m}_{e}^{*}$ have a ZT of 0.37 . This lead to a conclusion that intermediate band gap materials with low effective mass have a promising feature of equipping high ZT.

\subsection{Relaxation of the unit cell}

To estimate the spin and charge transport in cobalt spinel configurations using NEGF approximation, the fundamental parameters such as the fundamental band gap, effective mass of conduction electrons and net magnetization, have to be calculated using first principles approach. The unit cell coordinates were extracted from open source crystallography database, followed by relaxation of the forces of the atoms within the unit cell such that the total energy of the system attains least energy. This is based of the physical principle that a system is most stable in its lowest energy state.

In case of substitutions with $\mathrm{Ni}$ and $\mathrm{Zn}$ as discussed in Section 1.4, depending the amount of substitutions, $\mathrm{Ni}^{+2}$ or $\mathrm{Zn}^{+2}$ atoms prefer to occupy specific sites (tetrahedral or octahedral) in the spinel lattice. For instance in case of $\mathrm{Co}_{2.75} \mathrm{Zn}_{0.25} \mathrm{O}_{4}$, the 56 atom supercell 
formula can be written as $\mathrm{Co}_{16} \mathrm{Co}_{6} \mathrm{Zn}_{2} \mathrm{O}_{32}$. Here the two zinc atoms in $\mathrm{Co}_{2.75} \mathrm{Zn}_{0.25} \mathrm{O}_{4}$ configuration have 8 tetrahedral positions to substitute which yields 28 different combinations in the supercell. All 28 different combinations were relaxed and the combination which yielded lowest energy among all the combinations was chosen to further calculate the fundamental properties. A similar approach was applied for all configurations in the proposed design space where each configuration was tested with all possible combinations and the cell which yielded lowest energy was chosen to perform further analysis.

\subsection{Spin polarized calculations using density functional theory}

Density functional theory (DFT) is widely used to estimate the fundamental properties of material configurations which include band structure (effective mass, band gap), lattice parameter, magnetization and atom projected density of states. As the spin transport model treats both spin channels independently, the spin up and spin down band gaps were calculated. Figure 5.8 shows the atom projected density of states of pure $\mathrm{Co}_{3} \mathrm{O}_{4}$ spinel lattice. The states represented below the $\mathrm{x}$-axis are spin down states and those above $\mathrm{x}$-axis are spin up states. It can be observed that the band gap is different for spin up and spin down channels suggesting that both channels have different electronic conductivities and hence the respective charge carries (along with the spin associated with them) travel at a different rate under an external temperature bias.

The blue lines in the Figure 5.8 indicate the cobalt atoms at the tetrahedral sites. It can be observed that the valance and conduction states in spin down channel are mainly contributed by the cobalt atoms in tetrahedral sites. And also as the fundamental band gap of $\mathrm{Co}_{3} \mathrm{O}_{4}$ spinel is same as the band gap of spin down channel, it can be hypothesized that Co atoms at tetrahedral sites have high contribution towards conduction in the lattice. In addition, the spin polarization in the material lattice which is caused due to the difference 


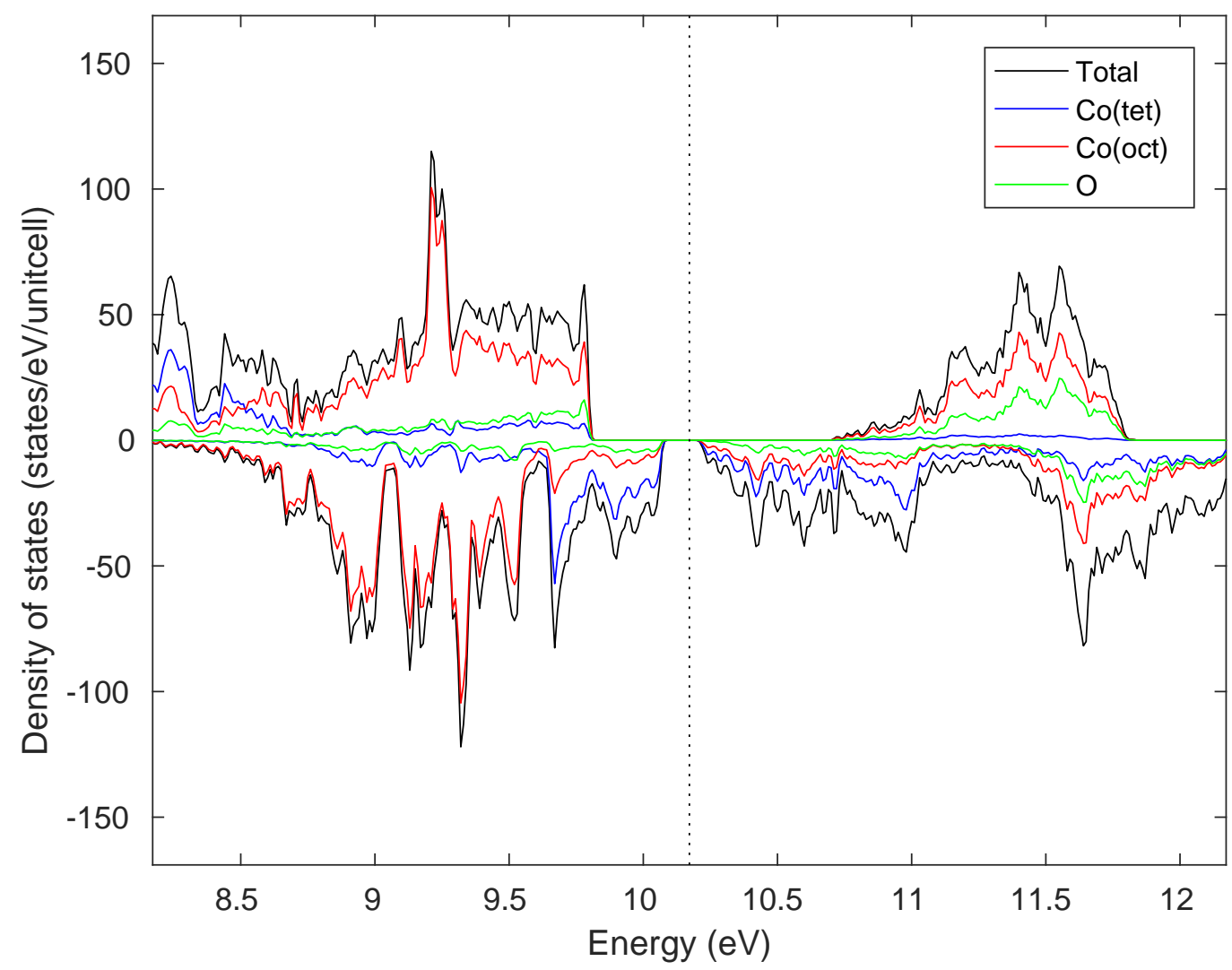

Figure 5.8 The atom projected density of states in $\mathrm{Co}_{3} \mathrm{O}_{4}$ spinel lattice. The Co atoms in the tetrahedral (tet) sites majorly contribute to the net spin polarization in the material due to their difference in the energy states while Co atoms in the Octahedral (oct) sites have minimal contribution towards spin polarization.

in the spin up and spin down populations is again due to the difference in the populations mainly from the cobalt atoms in the tetrahedral sites.

Through the above observations it is evident that tetrahedral sites in a spinel configuration are major contributors to the spin polarization. To strengthen this argument, the substitution of $\mathrm{Zn}$ in the tetrahedral sites to form the spinel configuration $\mathrm{Co}_{2.5} \mathrm{Zn}_{0.5} \mathrm{O}_{4}$, is shown in Figure 5.9. The majority of states near the fermi region are occupied by the cobalt atoms at the tetrahedral sites. Also, the spin polarization in $\mathrm{Co}_{2.5} \mathrm{Zn}_{0.5} \mathrm{O}_{4}$ is due to the spin population difference in the tetrahedral sites. 


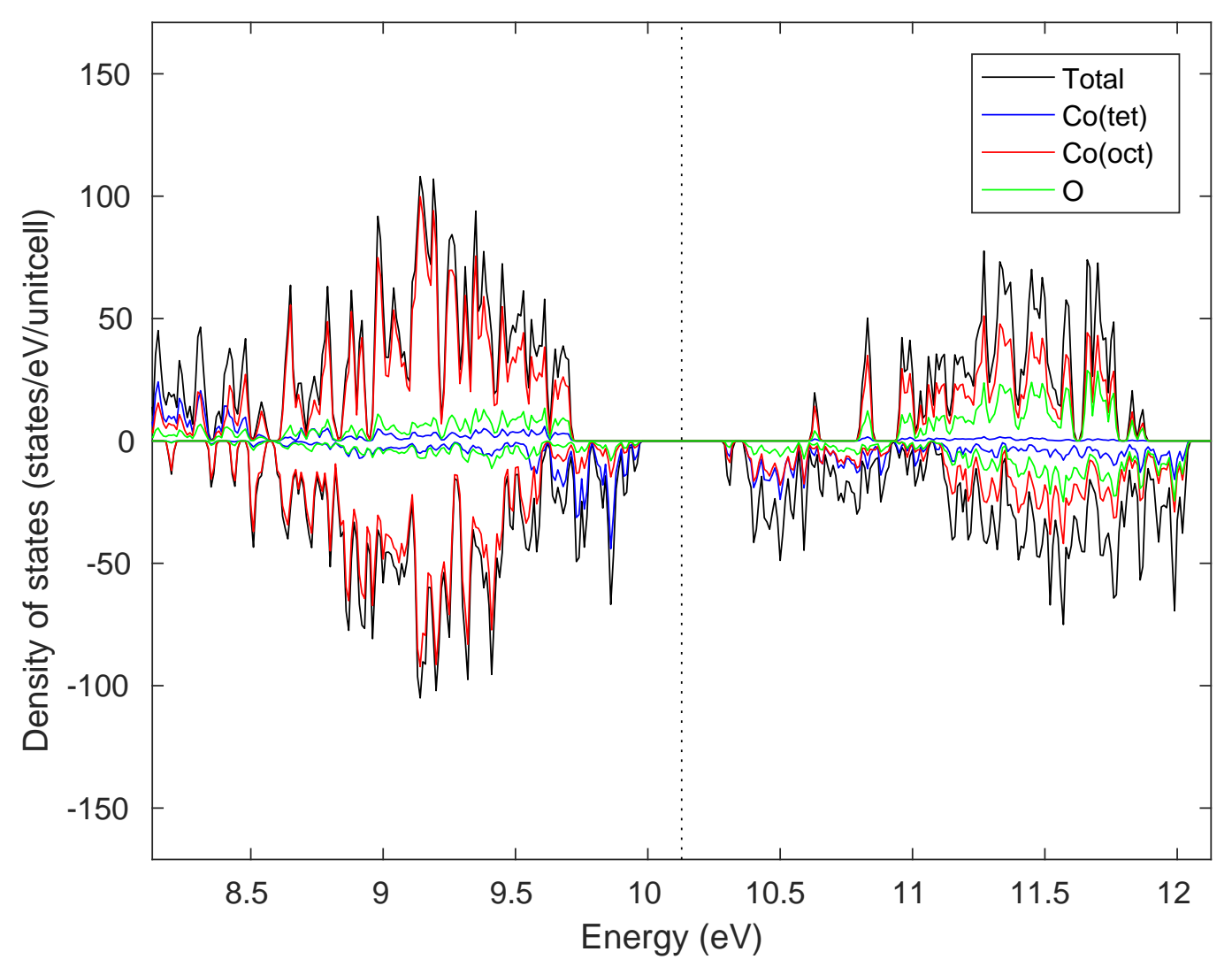

Figure 5.9 Atom projected density of states for $\mathrm{Co}_{2.5} \mathrm{Zn}_{0.5} \mathrm{O}_{4}$. Blue lines indicate the states due to cobalt atoms in the tetrahedral states that majorly influence the spin polarization and conductivity in a spinel matrix.

According to crystal field theory, zinc atom in a tetrahedral occupancy attains a d10 configuration as shown in Figure 5.10, which shows completely filled d orbitals. Due to this characteristic, the zinc atoms in a tetrahedral configuration donot contribute to spin polarization. This is evident from Figure 5.11, where all the eight tetrahedral sites were completely substituted with zinc atoms. Due to the d10 stable configuration, the electrons in d orbital of zinc donot contribute to conduction and hence there are near to zero states due to zinc tetrahedral sites near the Femi region. And also it is evident that the spin polarization is zero which strengthens the aurgument that atoms in tetrahedral sites in a 
spin lattice contribute to net spin polarization.

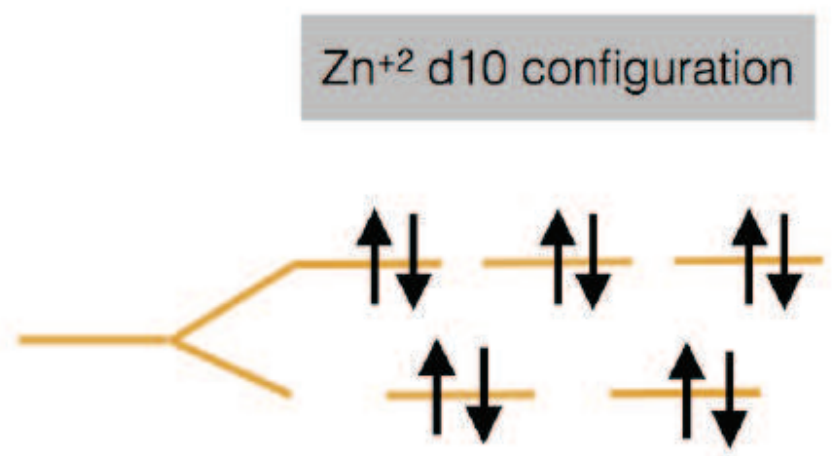

Figure 5.10 Crystal field splitting of $\mathrm{Zn}^{+2}$ in tetrahedral occupancy.

To study the effect of $\mathrm{Ni}^{+2}$ and $\mathrm{Zn}^{+2}$ substitutions, they were substituted in place of $\mathrm{Co}^{+2}$ on the tetrahedral sites which form normal spinel configuration. In the configurations that substituted both $\mathrm{Ni}^{+2}$ and $\mathrm{Zn}^{+2}$ in place of $\mathrm{Co}^{+2}$ in the tetrahedral sites, like $\mathrm{Co}_{2} \mathrm{Ni}_{0.25} \mathrm{Zn}_{0.75} \mathrm{O}_{4}$, the density of states are shown in Figure 5.12. It can be seen that this configuration must acts like a conductor with a zero band gap, but the electron density plot of this configuration, as shown in Figure 5.13, depicted the electron cloud localised only around the $\mathrm{Ni}^{+2}$ atoms in the tetrahedral sites. Though the density of states are present near the Fermi region, the localised electron density cloud present on specific atoms do not have interlinks with the rest of the cell and hence these states cannot provide a uniform conduction path inside the cell. So, these states were ignored while calculating the band gap of the spin channels.

In Figure 5.13, the electron density plot of $\mathrm{Co}_{2} \mathrm{Ni}_{0.25} \mathrm{Zn}_{0.75} \mathrm{O}_{4}$ is shown. Here, the 


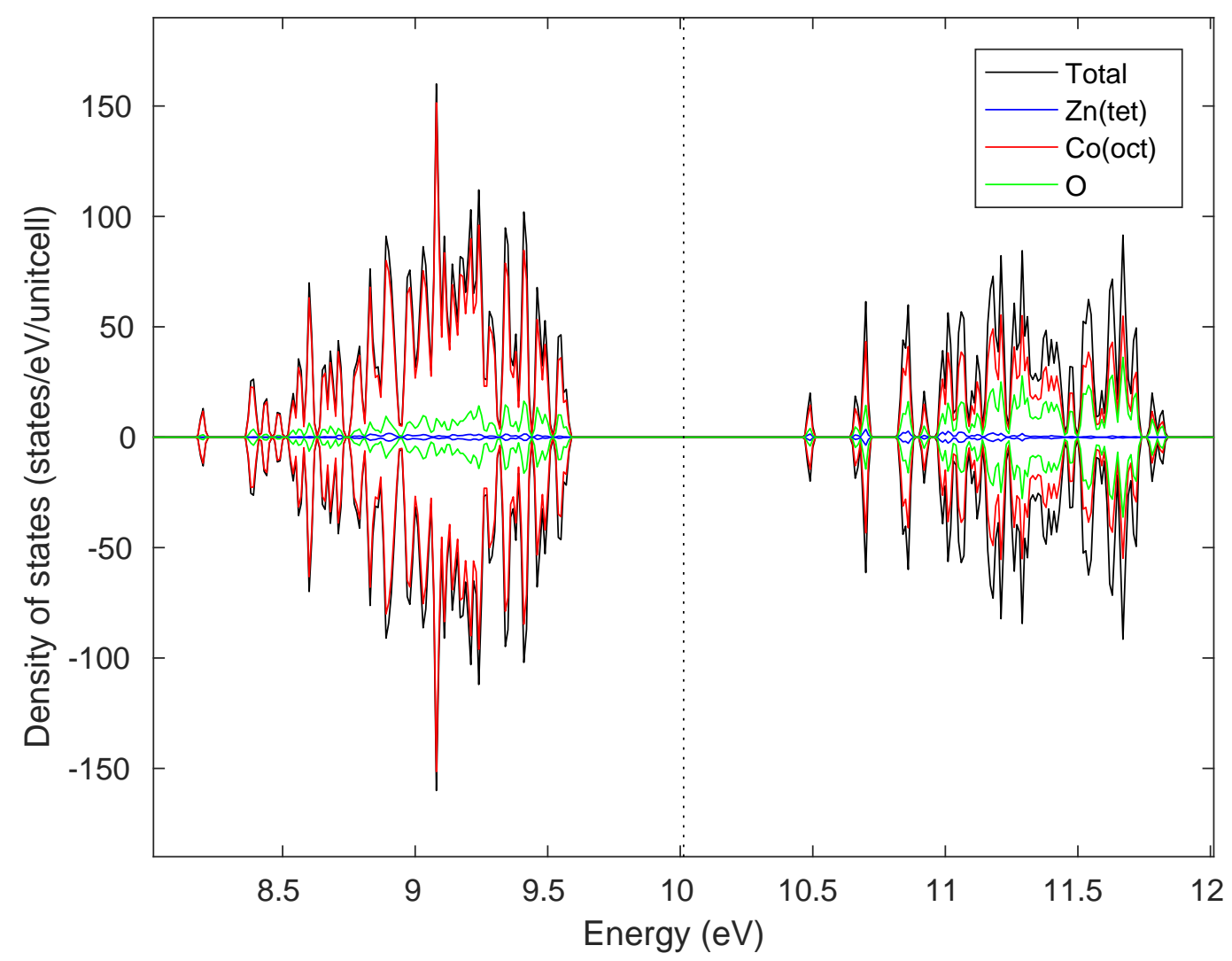

Figure 5.11 Density of states for $\mathrm{Co}_{2} \mathrm{ZnO}_{4}$. This spinel configuration shows no net spin polarization due to zero contribution from completely filled $\mathrm{Zn}^{+2}$ atoms occupying tetrahedral sites.

$0.25 \mathrm{eV}$ energy range is used to plot the electron density cloud in the material. The electron cloud is only localised around the Ni atoms and hence donot contribute to the over all electron conduction in the cell. Therefore in Figure 5.12, the localised energy states around the Fermi region can be associated only to Ni atoms and ignored as contributing factors to the overall electron conduction of $\mathrm{Co}_{2} \mathrm{Ni}_{0.25} \mathrm{Zn}_{0.75} \mathrm{O}_{4}$ material. Hence, the band gap of the material will be $0.78 \mathrm{eV}$ ignoring the localised energy levels in the DOS plot of $\mathrm{Co}_{2} \mathrm{Ni}_{0.25} \mathrm{Zn}_{0.75} \mathrm{O}_{4}$.

The band gaps of the spin up and spin down channels were calculated for the entire 


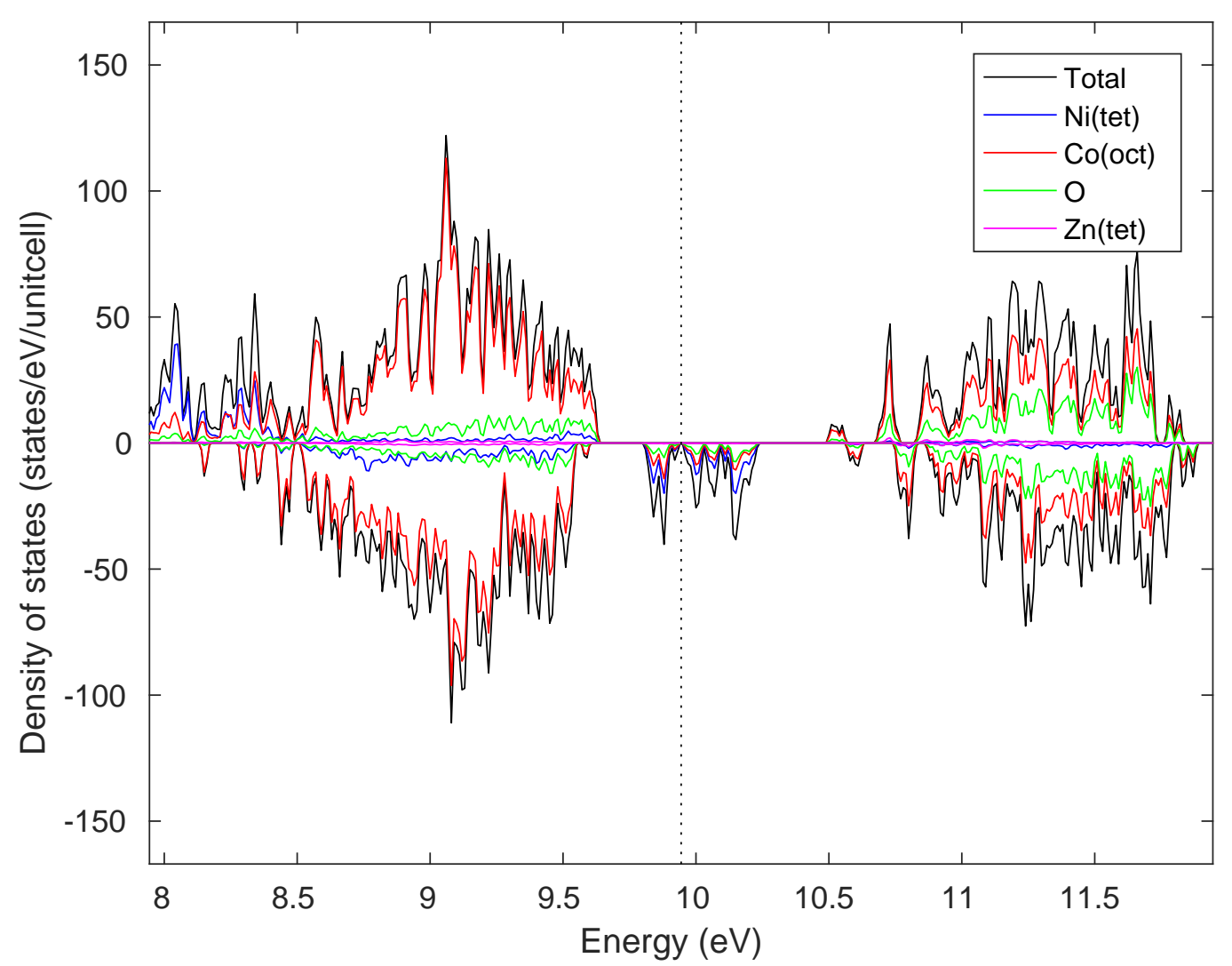

Figure 5.12 Density of states for $\mathrm{Co}_{2} \mathrm{ZnO}_{4}$. This spinel configuration shows no net spin polarization due to zero contribution from completely filled $\mathrm{Zn}^{+2}$ atoms occupying tetrahedral sites.

design space. The ternary plot depicts a trend of variation in band gap due to different substitutions along the borders and inside the design region. Spin down channels have relatively lower band gap and hence are relatively conductive than spin up channels. The low band gap of the spin down channels can be associated to the atoms present in the tetrahedral sites. Hubbard correction term was not implemented to these cases as the trend in the transport properties studied for configurations without $U$ along the design space, can have a similar trend when $\mathrm{U}$ correction is applied to all the configurations.

When a Co in tetrahedral site is substituted with a $\mathrm{Zn}$ or a $\mathrm{Ni}$, the band gap and fermi energy level shift towards the conduction or valance bands making them behave like a p-tpe or n-type materials. The individual band gaps of the two spin channels of the entire design 


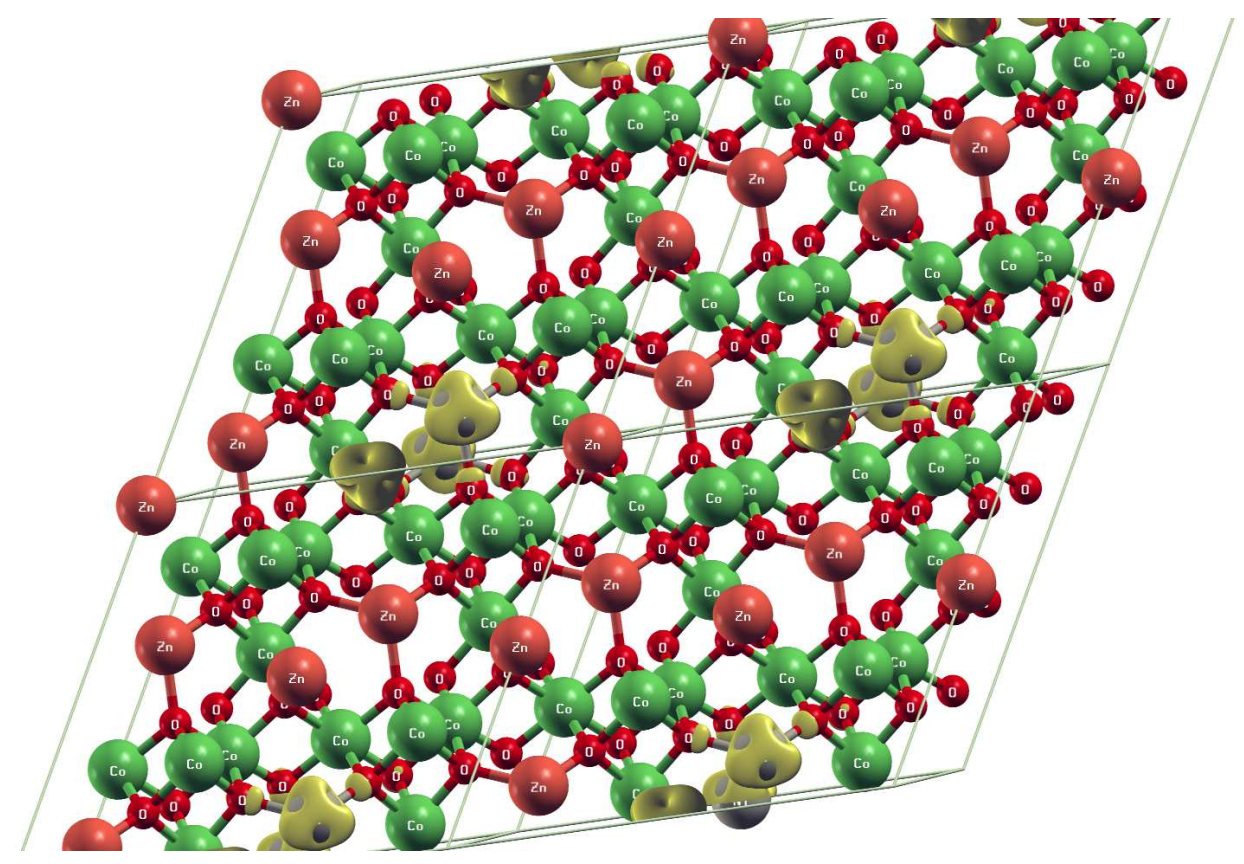

Figure 5.13 Electron density plot of the $\mathrm{Co}_{2} \mathrm{Ni}_{0.25} \mathrm{Zn}_{0.75} \mathrm{O}_{4}$ showing electron cloud localised around $\mathrm{Ni}$ atoms on the tetrahedral sites. The energy range within $0.25 \mathrm{eV}$ associates the localised energy states only to $\mathrm{Ni}$ atoms which donot form a unifom interlink through out the cell and hence they cannot contribute to electronic conduction within the cell.

space formed by substituting Zn and $\mathrm{Ni}$ atoms into the cobalt oxide spinel lattice is shown in Figure 5.14. It is evident from the Figure 5.14 that spin down channels have low band gap compared to the spin up channels. The trend of the current density of the entire configuration space for the substitutions of cobalt with zinc and nickel, the band gaps for which are as shown in the ternary plot given in Figure 5.14, is shown in Figure 5.16. Similarly, the electrical conductivity for the entire configuration space is shown in Figure 5.17. With the amount of Zn substitution, the active d orbital states became completely full causing the band gap to increase and hence resulting in the low electrical conductivity.

Along with the band gap, the curvature of the conduction band edge also influences the spin transport properties. The effective mass of the conduction electron depends on the curvature and k-point path. In order to attain effective mass of a conduction electron 

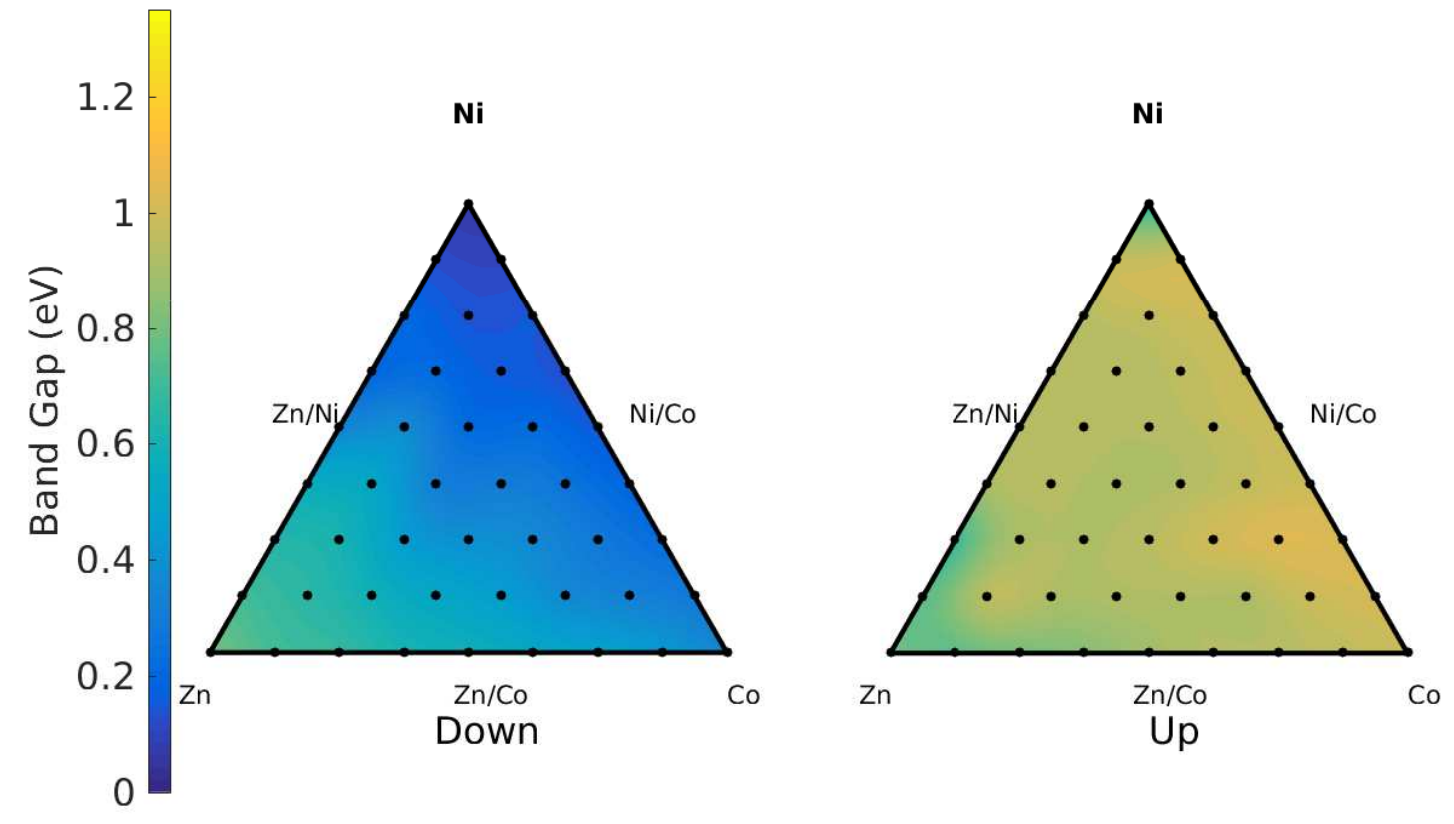

Figure 5.14 A ternary plot of spin up and spin down band gaps of different configurations in the design space.

the harmonic mean of effective mass considering three perpendicular directions has been calculated. Three perpendicular k-point path depends on the type of structure like FCC or BCC etc. Spinel lattice investigated in this research is an FCC strucuture. From the Brillouin zone of FCC, the three k-points paths perpendicular to one another centered at $\Gamma$ point are $\mathrm{X}$ to $\Gamma, \Gamma$ to $\mathrm{L}$ and $\Gamma$ to $\mathrm{N}$ where $\mathrm{X}$ is $\left(\begin{array}{lll}0.5 & 0.5 & 0.0\end{array}\right), \Gamma$ is $\left(\begin{array}{lll}0 & 0 & 0\end{array}\right), \mathrm{L}$ is $\left(\begin{array}{lll}0.0 & 0.5 & 0.0\end{array}\right)$ and $\mathrm{N}$ is $\left(\begin{array}{lll}0.5 & 0.5 & 0.5\end{array}\right)$.

Figure 5.15 shows the conduction and valence bands of $\mathrm{Co}_{3} \mathrm{O}_{4}$. For this band structure, the effective mass of the conduction band electrons were calculated. A similar approach is followed for the entire design space as shown in Figure 5.14 and for each of the structures, the mean effective mass has been calculated and tabulated in the Tables 5.2, 5.3 and 5.4 for 


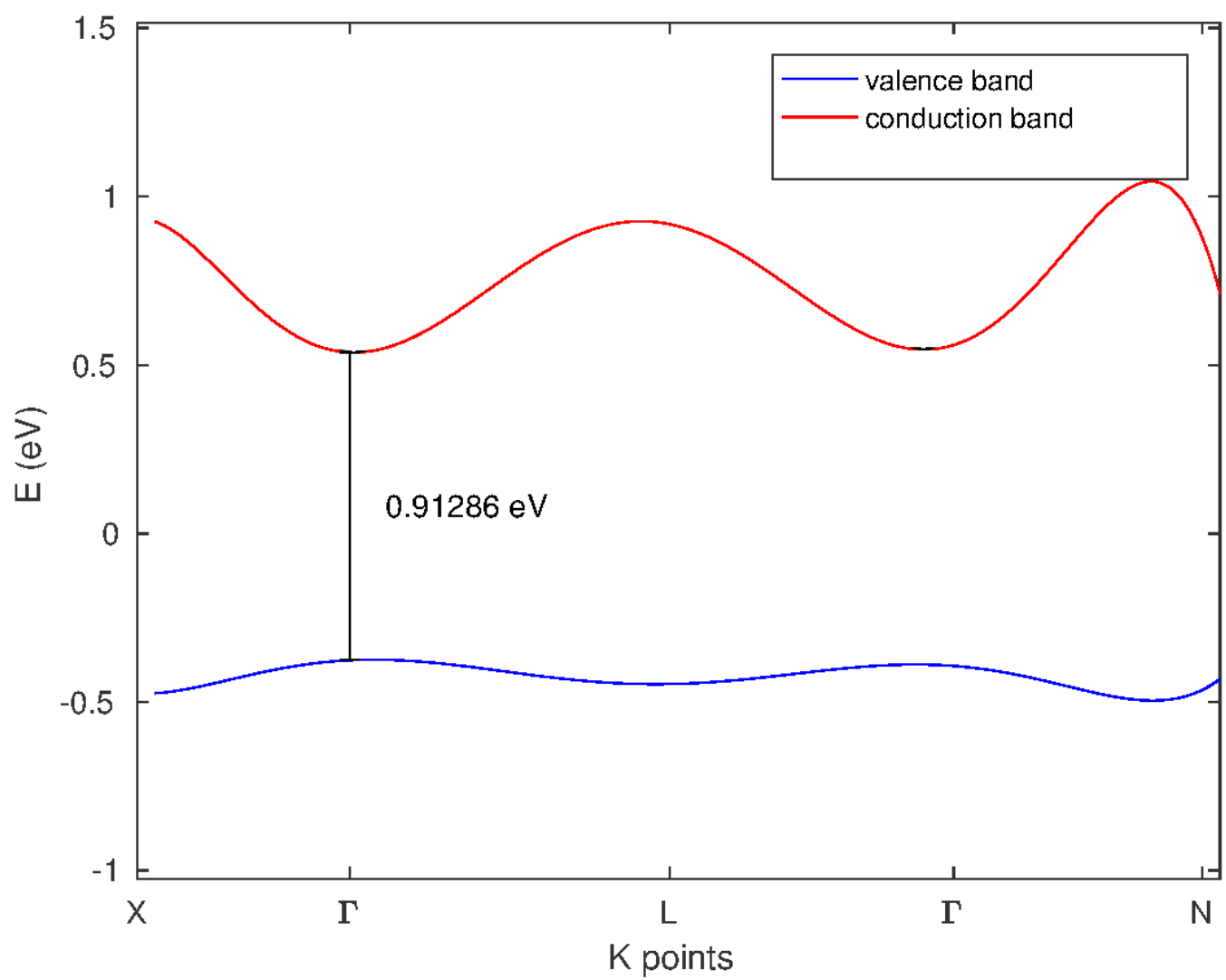

Figure 5.15 Spin up conduction and valance bands of $\mathrm{Co}_{3} \mathrm{O}_{4}$. The three effective mass $\mathrm{m}_{x}, \mathrm{~m}_{y}$ and $\mathrm{m}_{z}$ are calculated for three paths $\mathrm{X}$ to Gamma, $\Gamma$ to $\mathrm{L}$ and $\Gamma$ to $\mathrm{N}$ respectively. The calculated effective mass curves are plotted on the conduction band. This band structure plot gives the information about the band gap between conduction and valance band edges and the three effective mass.

$\mathrm{Co}_{3-x} \mathrm{Zn}_{x} \mathrm{O}_{4}, \mathrm{Co}_{3-x} \mathrm{Ni}_{x} \mathrm{O}_{4}$ and $\mathrm{Co}_{2} \mathrm{Ni}_{1-y} \mathrm{Zn}_{y} \mathrm{O}_{4}$ respectively where $0<\mathrm{y}<1$. 
Table 5.2 The band gap, effective mass of spin up and spin down conduction channels and, the magnetic moment of each configuration along the $0<\mathrm{x}<1 \mathrm{Co}_{3-x} \mathrm{Zn}_{x} \mathrm{O}_{4}$.

\begin{tabular}{|c|c|c|c|c|c|}
\hline \multicolumn{6}{|c|}{ Cell parameter with no spin polarization } \\
\hline Configuration & \multicolumn{2}{|c|}{ Band gap } & \multicolumn{2}{|c|}{ Effective mass ${ }^{*} \mathrm{~m}_{e}$} & Magnetic moment \\
\hline & spin up & spin down & spin up & spin down & in Bohr-Magneton \\
\hline $\mathrm{Co}_{3} \mathrm{O}_{4}$ & 0.912 & 0.35 & 1.5 & 3.51 & 6.0 \\
\hline $\mathrm{CO}_{2.75} \mathrm{Zn}_{0.25} \mathrm{O}_{4}$ & 0.936 & 0.49 & 2.33 & 3.23 & 4.1 \\
\hline $\mathrm{Co}_{2.5} \mathrm{Zn}_{0.5} \mathrm{O}_{4}$ & 0.89 & 0.58 & 1.02 & 2.84 & 3.0 \\
\hline $\mathrm{Co}_{2.25} \mathrm{Zn}_{0.75} \mathrm{O}_{4}$ & 0.8 & 0.68 & 1.12 & 2.31 & 1.5 \\
\hline $\mathrm{Co}_{2} \mathrm{ZnO}_{4}$ & 0.80 & 0.80 & 1.97 & 1.97 & 0 \\
\hline
\end{tabular}

Table 5.3 The band gap, effective mass of spin up and spin down conduction channels and, the magnetic moment of each configuration along the $0<\mathrm{x}<1 \mathrm{Co}_{3-x} \mathrm{Ni}_{x} \mathrm{O}_{4}$.

\begin{tabular}{|c|c|c|c|c|c|}
\hline \multicolumn{5}{|c|}{ cell parameter, band gap and spin polarization } \\
\hline Configuration & \multicolumn{2}{|c|}{ Band gap } & \multicolumn{2}{c|}{ Effective mass* ${ }_{e}$} & Magnetic moment \\
\hline & spin up & spin down & spin up & spin down & in Bohr-Magneton \\
\hline $\mathrm{Co}_{3} \mathrm{O}_{4}$ & 0.912 & 0.35 & 1.5 & 3.51 & 6.0 \\
\hline $\mathrm{Co}_{2.75} \mathrm{Ni}_{0.25} \mathrm{O}_{4}$ & 0.9 & 0.23 & 0.358 & 5.36 & 4.52 \\
\hline $\mathrm{Co}_{2.5} \mathrm{Ni}_{0.5} \mathrm{O}_{4}$ & 0.94 & 0.15 & 1.56 & 2.03 & 6.23 \\
\hline $\mathrm{Co}_{2.25} \mathrm{Ni}_{0.75} \mathrm{O}_{4}$ & 0.94 & 0.15 & 1.01 & 3.29 & 6.81 \\
\hline $\mathrm{Co}_{2} \mathrm{NiO}_{4}$ & 0.95 & 0.07 & 0.95 & 1.65 & 6.86 \\
\hline
\end{tabular}

Table 5.4 The band gap, effective mass of spin up and spin down conduction channels and, the magnetic moment of each configuration along the $0<\mathrm{y}<1 \mathrm{Co}_{2} \mathrm{Ni}_{1-y} \mathrm{Zn}_{y} \mathrm{O}_{4}$.

\begin{tabular}{|c|c|c|c|c|c|}
\hline \multicolumn{7}{|c|}{ cell parameter, band gap and spin polarization } \\
\hline Configuration & \multicolumn{2}{|c|}{ Band gap } & \multicolumn{2}{c|}{ Effective mass ${ }^{*} \mathrm{~m}_{e}$} & Magnetic moment \\
\hline & spin up & spin down & spin up & spin down & in Bohr-Magneton \\
\hline $\mathrm{Co}_{3} \mathrm{O}_{4}$ & 0.912 & 0.16 & 1.5 & 10.68 & 6.0 \\
\hline $\mathrm{Co}_{2} \mathrm{Ni}_{0.25} \mathrm{Zn}_{0.75} \mathrm{O}_{4}$ & 0.84 & 0.95 & 0.32 & 4.33 & 1.0 \\
\hline $\mathrm{Co}_{2} \mathrm{Ni}_{0.5} \mathrm{Zn}_{0.5} \mathrm{O}_{4}$ & 0.88 & 1.09 & 0.62 & 3.02 & 2.0 \\
\hline $\mathrm{Co}_{2} \mathrm{Ni}_{0.75} \mathrm{Zn}_{0.25} \mathrm{O}_{4}$ & 0.89 & 1.19 & 1.51 & 5.21 & 3.0 \\
\hline $\mathrm{Co}_{2.25} \mathrm{Ni}_{0.25} \mathrm{Zn}_{0.5} \mathrm{O}_{4}$ & 0.88 & 0.51 & 0.98 & 1.02 & 2.5 \\
\hline $\mathrm{Co}_{2.25} \mathrm{Ni}_{0.5} \mathrm{Zn}_{0.25} \mathrm{O}_{4}$ & 0.88 & 0.78 & 0.25 & 2.33 & 3.5 \\
\hline $\mathrm{Co}_{2.5} \mathrm{Ni}_{0.25} \mathrm{Zn}_{0.25} \mathrm{O}_{4}$ & 0.88 & 0.78 & 1.23 & 3.04 & 4.0 \\
\hline $\mathrm{Co}_{2.75} \mathrm{Ni}_{0.125} \mathrm{Zn}_{0.125} \mathrm{O}_{4}$ & 0.28 & 0.94 & 0.91 & 5.54 & 4.01 \\
\hline
\end{tabular}




\subsection{Spin Transport results}

With the available data of the fundamental properties of different configurations in the proposed design space formed by $\mathrm{Co}_{3} \mathrm{O}_{4}, \mathrm{Co}_{2} \mathrm{NiO}_{4}$ and $\mathrm{Co}_{2} \mathrm{ZnO}_{4}$, as tabultated in Tables 5.2, 5.3 and 5.4, the spin transport properties were investigated. Initially the conventional Seebeck, electrical conductivity and thermal conductivity of all spinel materials were calculated and then the improvement in the properties by extracting spin contribution to electrons was studied.

Figures 5.16, 5.17, 5.18, 5.19, 5.20, 5.21, are the conventional transport properties. The data from Table 5.2 shows that spin up channels have higher band gap compared to the spin down channels resulting in high Seebeck coefficient values of the spin up channels. This trend infers that the voltage generated by a spin channel that has higher band gap, will yield in high Seebeck coefficients as that can be observed in Figure 5.19.

It has to be noted that the net spin polarization in the configuration that has been completely substituted by zinc, $\mathrm{Co}_{2} \mathrm{ZnO}_{4}$, in tetrahedral sites is zero. This results in same band gap for both the spin channels. Hence, the voltage generated by both spin channels are the same. In addition the Figure 5.19 also presents the values of Seebeck coefficient of the focus region in the design space that comprises $\mathrm{Co}_{2.25} \mathrm{Ni}_{0.75} \mathrm{Zn}_{0.25} \mathrm{O}_{4}$. As $\mathrm{Co}_{2.25} \mathrm{Ni}_{0.75} \mathrm{Zn}_{0.25} \mathrm{O}_{4}$ displayed improved spin Seebeck properties, the results and discussion would follow, each trend also includes the respective data for $\mathrm{Co}_{2.25} \mathrm{Ni}_{0.75} \mathrm{Zn}_{0.25} \mathrm{O}_{4}$ to draw a comparison between the rest of the configurations with the optimal material in the focus region.

Due to the increase in the band gap, the conductivity of $\mathrm{Co}_{2-x} \mathrm{Zn}_{x} \mathrm{O}_{4}$ line decreased with the substitutions of $\mathrm{Zn}^{+2}$ in the tetrahedral sites, as shown in Figure 5.17. It has to be observed that the electrical conductivity of both the spin channels of $\mathrm{Co}_{2.25} \mathrm{Ni}_{0.75} \mathrm{Zn}_{0.25} \mathrm{O}_{4}$ have high conductivity while still maintaining a difference in their values by a factor of 10 , which results in a different diffusion rates in each of the spin channels. 


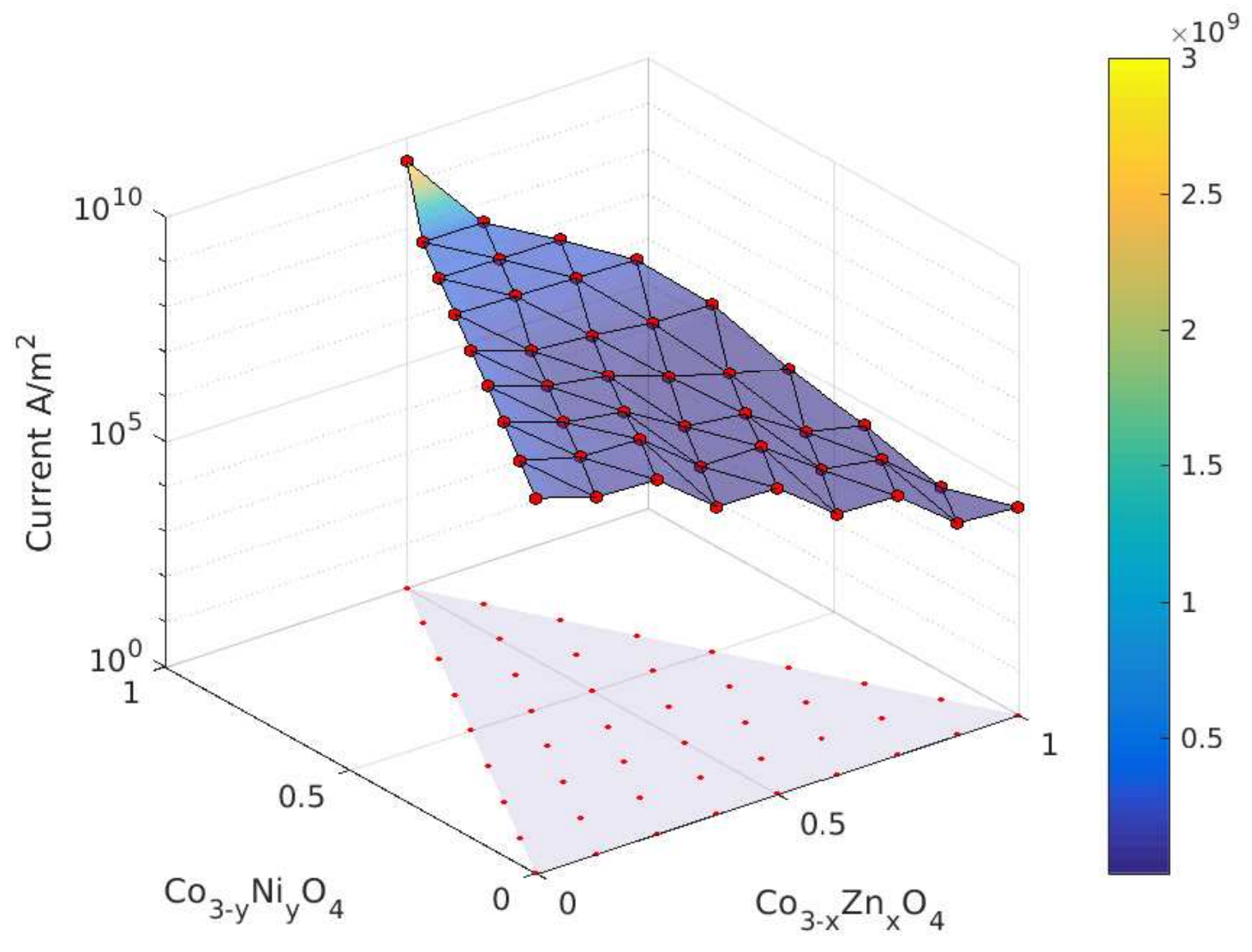

Figure 5.16 Current in $\mathrm{A} / \mathrm{m}^{2}$ for the configuraion space. The $\mathrm{x}$-axis shows $\mathrm{Co}_{2-x} \mathrm{Zn}_{x} \mathrm{O}_{4}$.

In case of $\mathrm{Ni}$ substituted $\mathrm{Co}_{3} \mathrm{O}_{4}$ giving rise to $\mathrm{Co}_{2-x} \mathrm{Ni}_{x} \mathrm{O}_{4}$ as shown in Figure 5.19, the overall Seebeck coefficient decreased with the $\mathrm{Ni}^{+2}$ substitution on the tetrahedral of $\mathrm{Co}_{2-x} \mathrm{Ni}_{x} \mathrm{O}_{4}$. The substitution of $\mathrm{Ni}^{+2}$ makes the material conductive as shown in Figure 5.17. Both the spin channels become conductive compared to the pure $\mathrm{Co}_{3} \mathrm{O}_{4}$. This is due to the interplay between effective mass and band gap in the material. Though the band gap remains nearly the same, low effective mass makes the channel more conductive and hence decreasing the Seebeck coefficient. The spin down channel of $\mathrm{Ni}^{+2}$ substituted $\mathrm{Co}_{3} \mathrm{O}_{4}$ has higher electric conductivity compared to both spin channels in $\mathrm{Co}_{2.25} \mathrm{Ni}_{0.75} \mathrm{Zn}_{0.25} \mathrm{O}_{4}$. While the Seebeck Coefficient is around $800 \mu \mathrm{V} / \mathrm{K}$ in the spin up channel of $\mathrm{Co}_{2.25} \mathrm{Ni}_{0.75} \mathrm{Zn}_{0.25} \mathrm{O}_{4}$. 


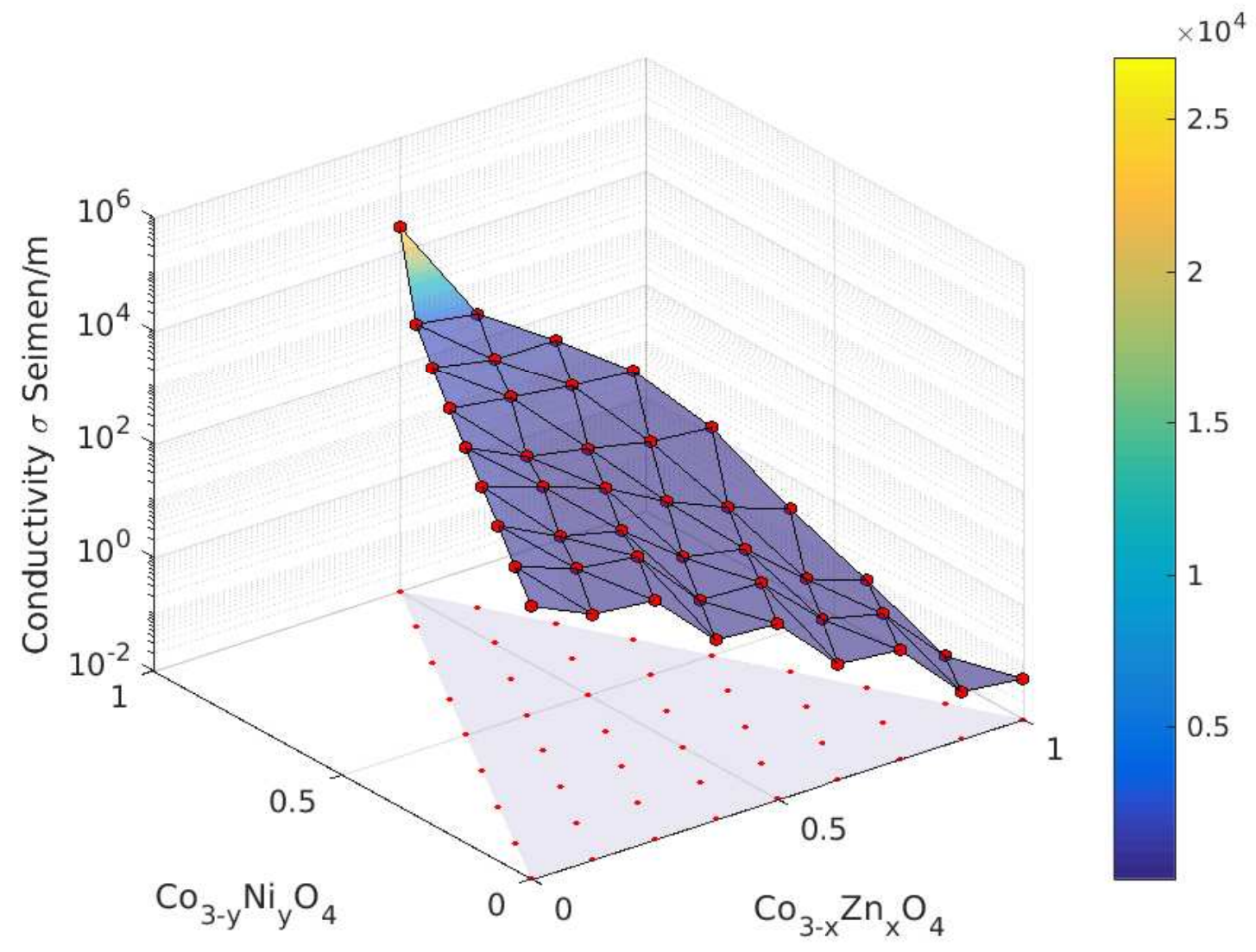

Figure 5.17 Electronic conductivity of the cobalt spinels, in Seimen/m for the configuraion space. High mobility associated with Ni substituted spinels has a high electronic conductivity and high band gap of $\mathrm{Zn}$ substituted cobalt spinels have low electronic conductivity.

$$
Z T_{\text {overall }}=2 *\left[\frac{1}{Z T_{\text {up }}}+\frac{1}{Z T_{\text {down }}}\right]^{-1}
$$

The power factor which is $S^{2} \sigma$, where $\mathrm{S}$ is the Seebeck coefficient and $\sigma$ is the electrical conductivity, decreases with $\mathrm{Zn}^{+2}$ substitution. While the power factor increases due to $\mathrm{Ni}^{+2}$ substitution. While the power factor of spin up channel $\mathrm{Co}_{2.25} \mathrm{Ni}_{0.75} \mathrm{Zn}_{0.25} \mathrm{O}_{4}$ is less when compared to $\mathrm{Ni}^{+2}$ substitution, the conventional $\mathrm{ZT}$ is higher for $\mathrm{Co}_{2.25} \mathrm{Ni}_{0.75} \mathrm{Zn}_{0.25} \mathrm{O}_{4}$ due low thermal conductivity in $\mathrm{Co}_{2.25} \mathrm{Ni}_{0.75} \mathrm{Zn}_{0.25} \mathrm{O}_{4}$ when compared to $\mathrm{Co}_{2} \mathrm{NiO}_{4}$ as shown in Figure 5.21. The $\mathrm{ZT}$ of $\mathrm{Co}_{2} \mathrm{NiO}_{4}$ is relatively higher than $\mathrm{ZT}$ of $\mathrm{Co}_{2} \mathrm{ZnO}_{4}$, given in Fig- 


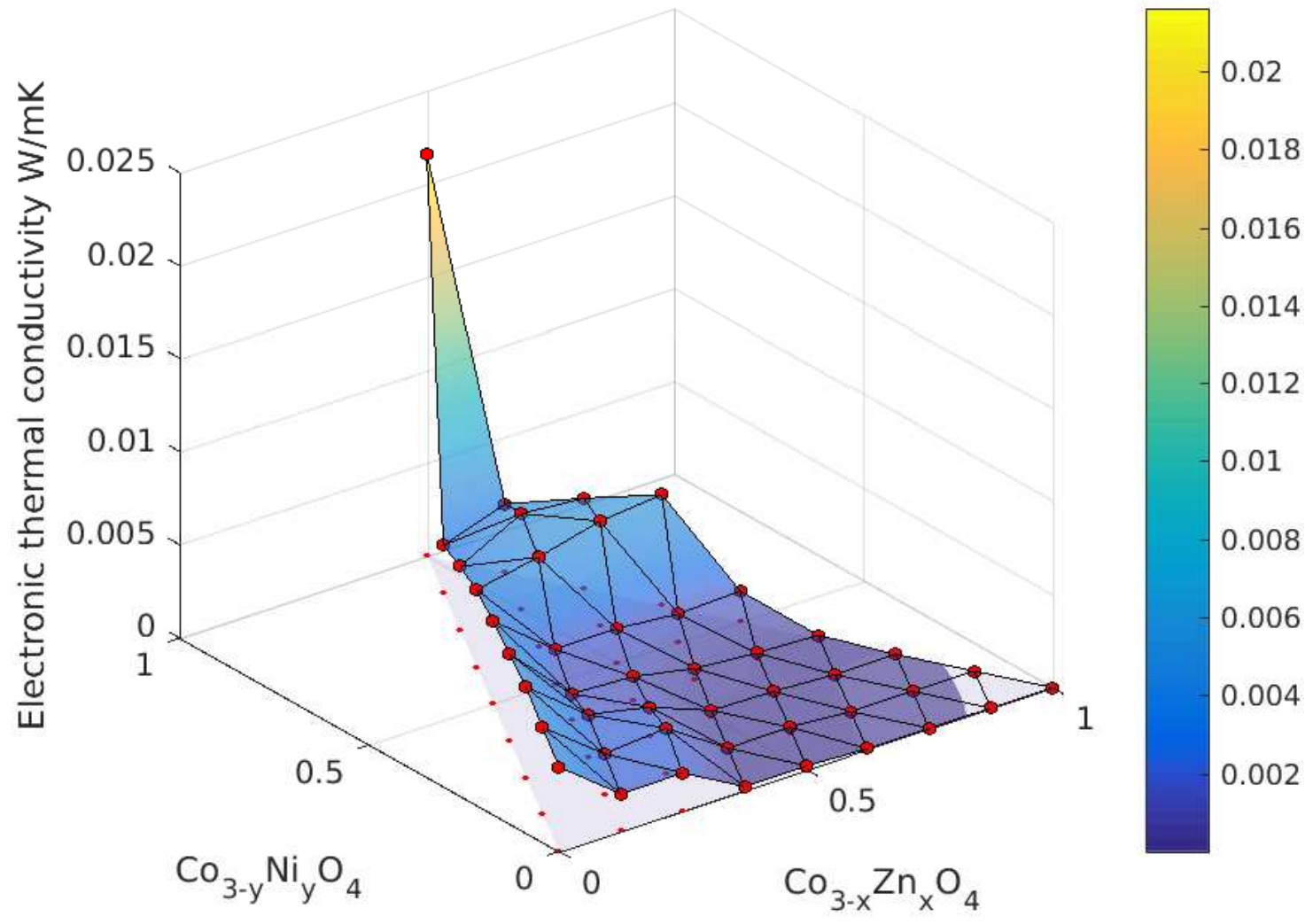

Figure 5.18 Electronic thermal conductivity of the cobalt spinels, in Seimen/m for the configuraion space. High mobility associated with Ni substituted spinels has a high electronic conductivity and electron contribution to thermal conductivity.

ure 5.21.

The conventional ZT data presents that zinc substitution in the cobalt spinels increase the Seebeck coefficient, while nickel substitution increases the electric conductivity. Hence, the focus region comprising $\mathrm{Co}_{2.25} \mathrm{Ni}_{0.75} \mathrm{Zn}_{0.25} \mathrm{O}_{4}$ is expected to have better thermoelectric properties. The overall ZT is calculated by the harmonic mean approach given by the Equation 5.1.

To increase the overall ZT, an additional spin voltage can be extracted using the inverse spin Hall effect. By employing the spin transport theory the additional voltage gen- 


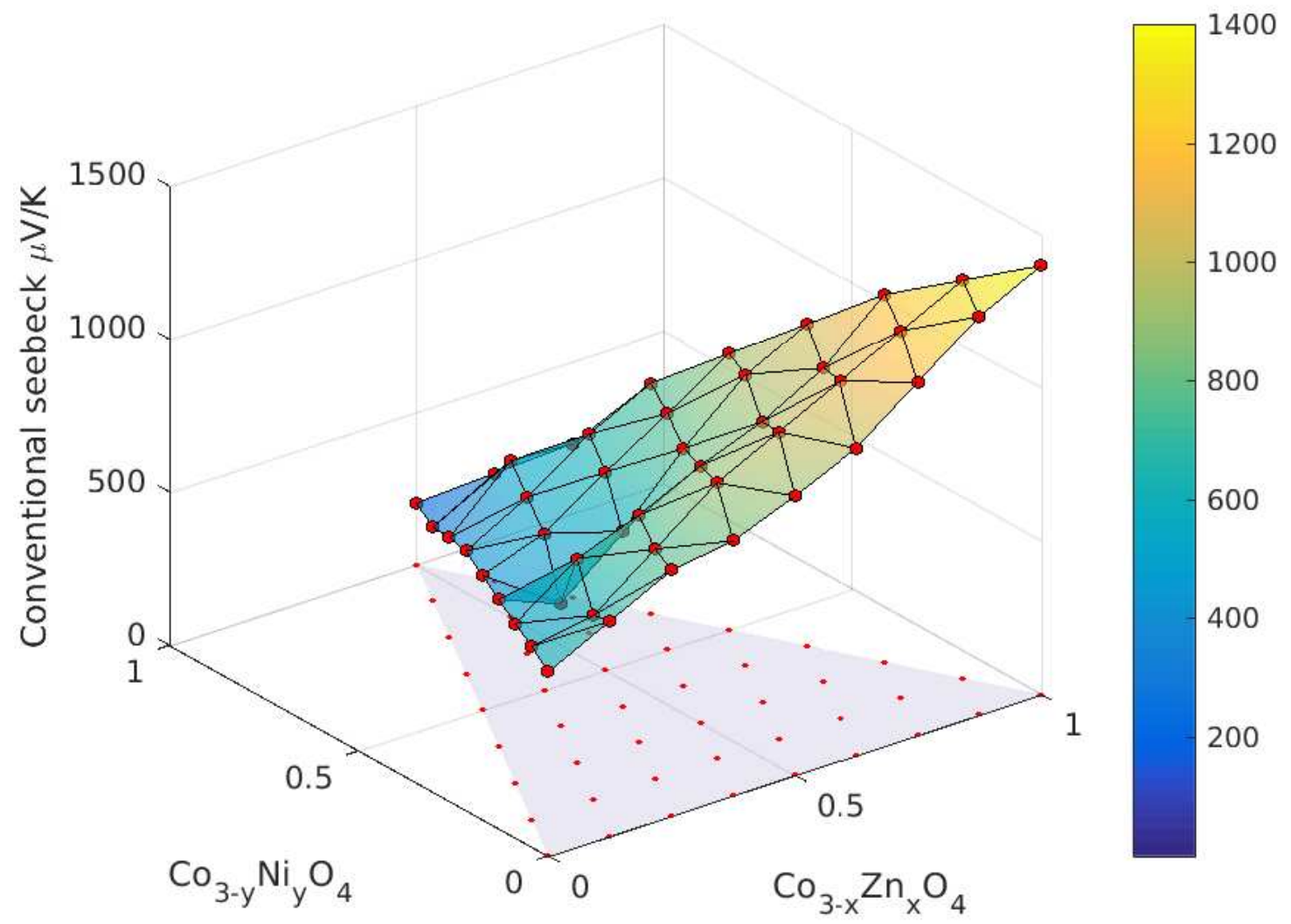

Figure 5.19 The Seebeck coefficient is proportional to the band gap in the material. Higer band gap associated to Zn substituted spinel shows a high Seebeck coefficient. Ni substituted cobalt spinel has the least band gap and hence has a low Seebeck coefficient.

erated purely depends on the difference in the spin populations along the magnetic material lattice. For instance, considering $\mathrm{Co}_{2.5} \mathrm{Zn}_{0.5} \mathrm{O}_{4}$, in addition to the conventional voltage of $0.03 \mathrm{~V}$, a spin voltage of $0.02 \mathrm{~V}$ can be extracted from the spinel lattice at $2 \mathrm{~nm}$ from the left end as shown in Figure 5.22. This additional voltage can be added to the conventional voltage generated in the matrial thereby increasing the overall Seebeck coefficient of the device and in overall $\mathrm{ZT}$ of the thermoelectric material.

Incorporating the additional spin Hall voltage, the overall ZT can be incresed by almost 5 folds as shown in Figure 5.23 and by more than 10 folds as shown in Figure 5.23. $\mathrm{Ni}$ is 


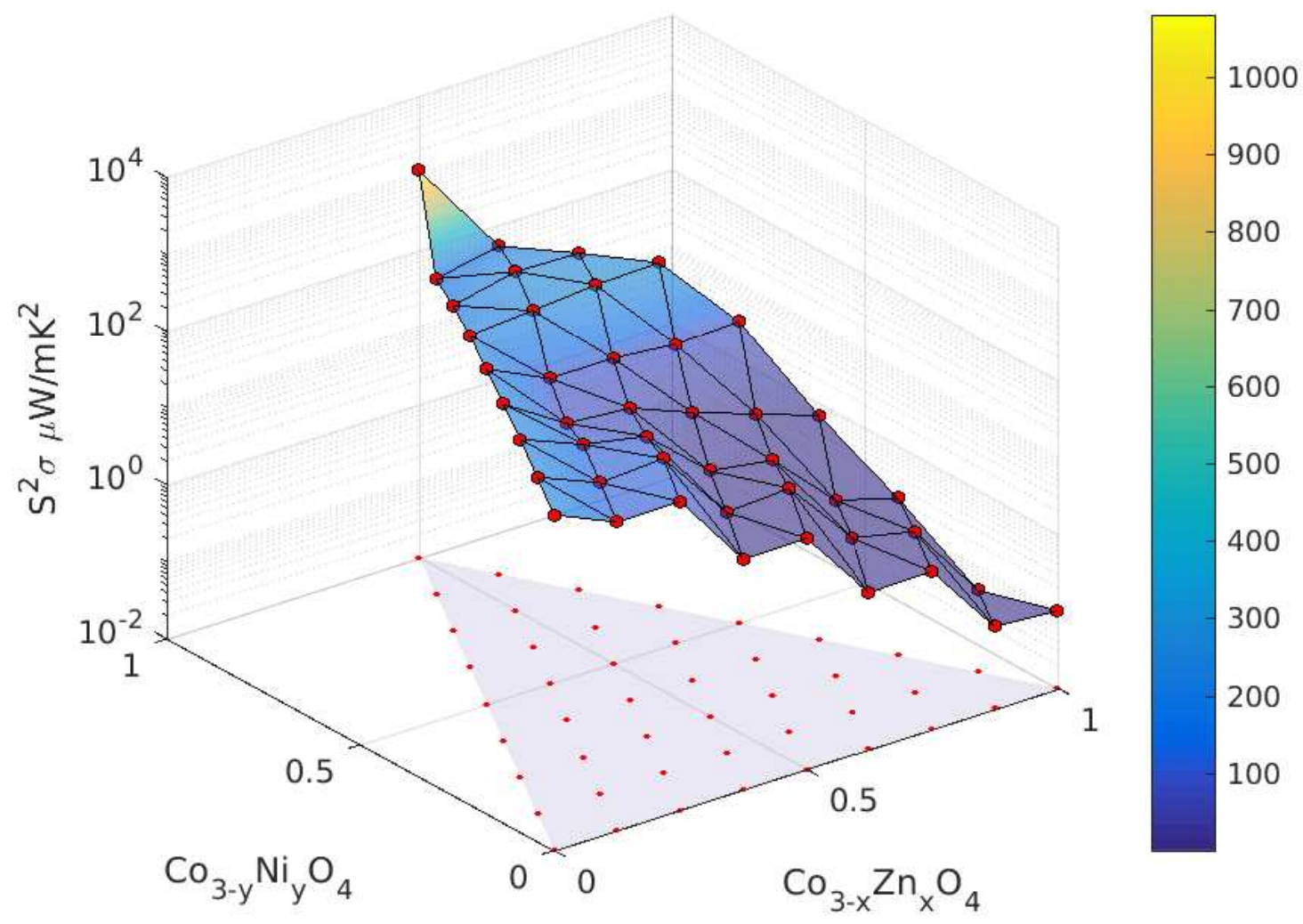

Figure 5.20 The power factor of the spinel configuration space. Power factor is directly proportional to the electrical conductivity of the material. Here, Ni substitution in the lattice showed the highest electrical conductivity and hence the power factor is highest for $\mathrm{Ni}$ occupation in tetrahedral sittes.

known for its property to induce spin polarization due to its incompletely filled d-orbitals and high spin configuration in the tetrahedral sites. Hence, higher spin Hall voltage can be extracted from the spinel lattice substituted with $\mathrm{Ni}$.

Hence, combining spin voltage and charge voltage in a thermoelectric material, the Seebeck coefficient can be increased. As, ZT of a thermoelectric material is proportional to the square of Seebeck coefficient, many folds increase in the performance can be possible. Thus, spin polarized materials have high scope in future thermoelectrics. 


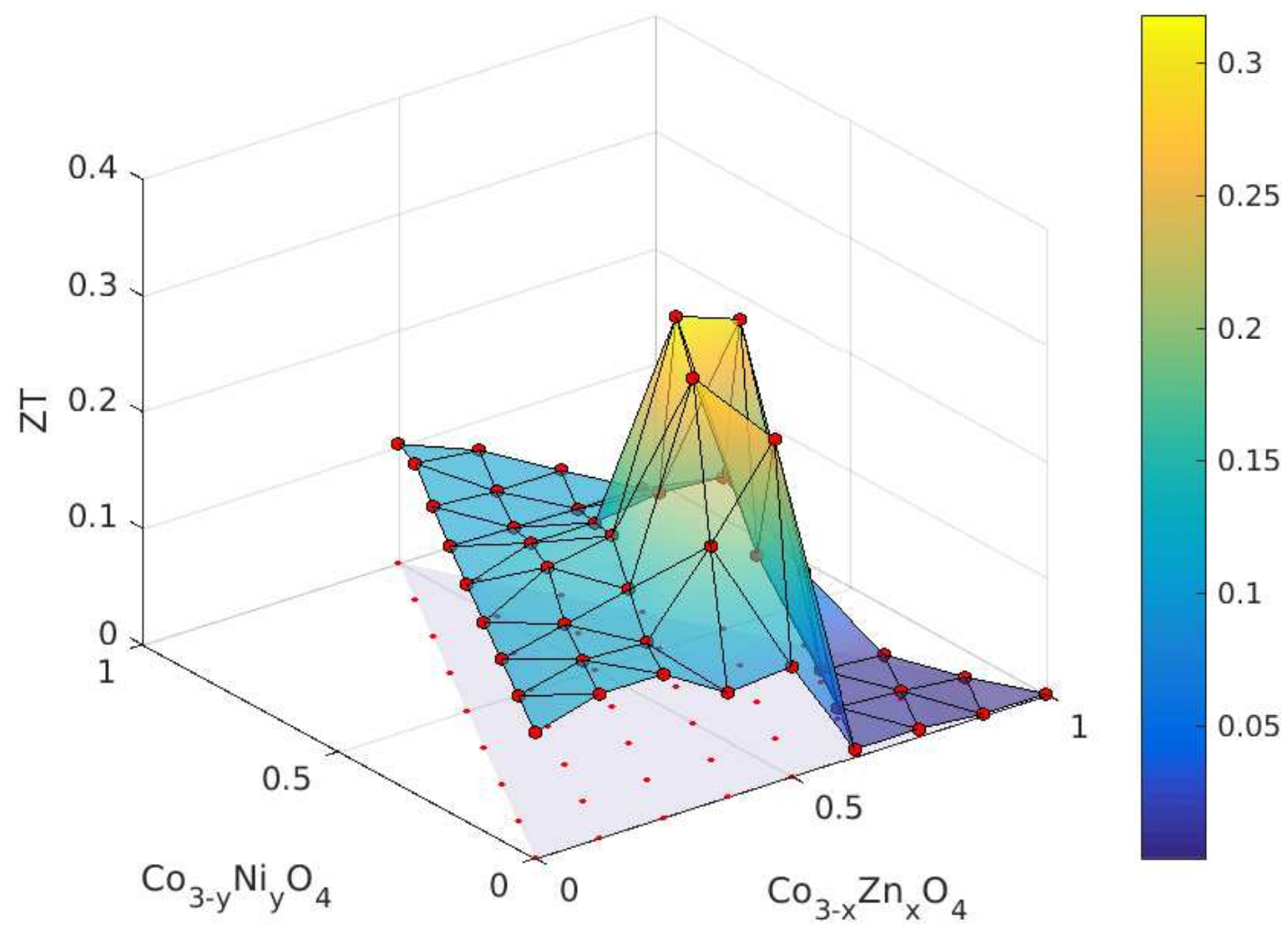

Figure 5.21 The thermoelectric performance of spinel oxides. The end configurations have a maximum ZT value of 0.15 , but the intermediate configurations having Ni and Zn substituted in the spinel lattice show an increase in the $\mathrm{ZT}$ to a value 0.3 . 


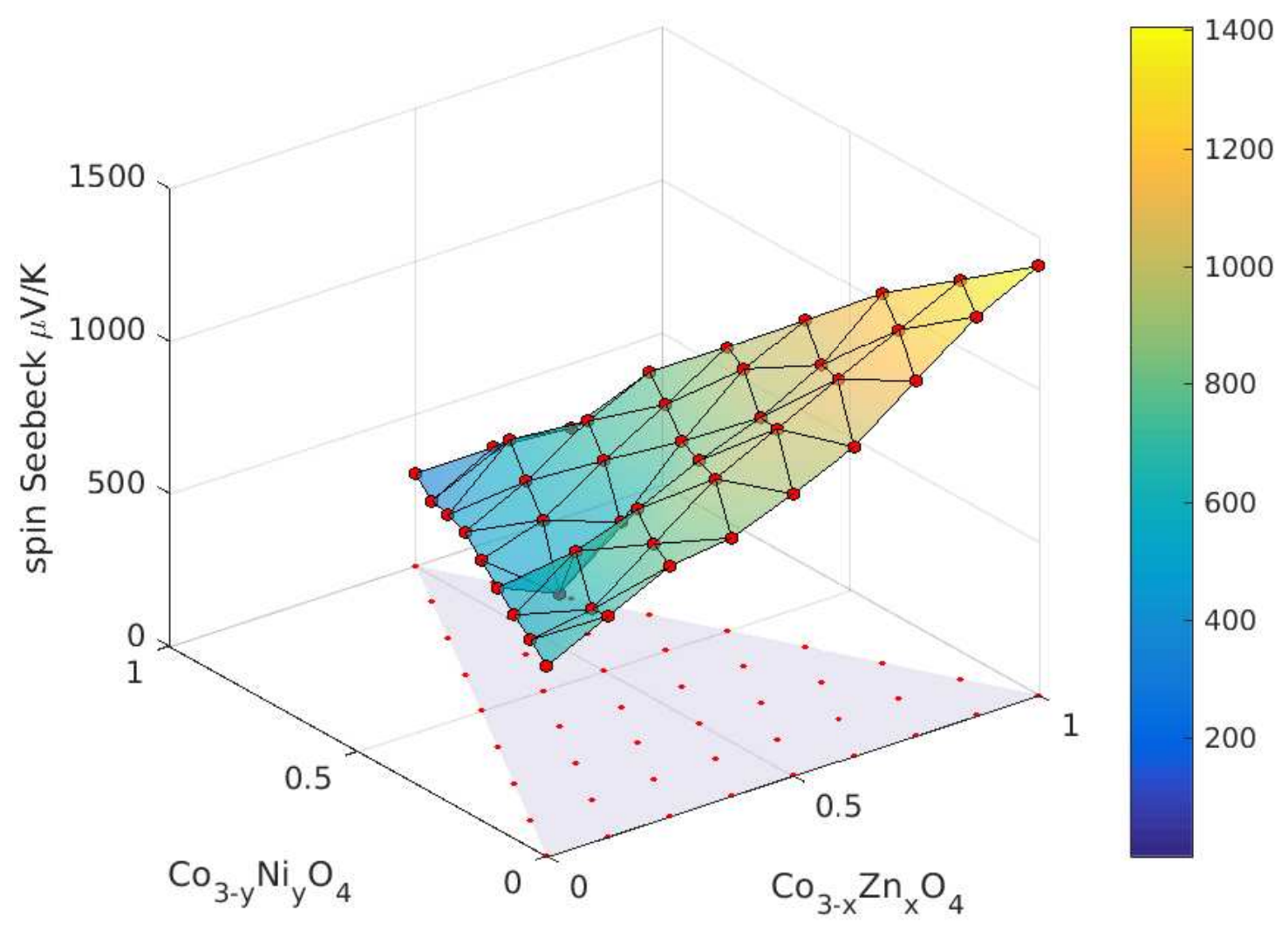

Figure 5.22 The total voltage output from the cell increases as the Seebeck coefficient increases. The inverse spin Hall voltage is improved due to the magnetization and the increase in the difference between the two spin populations. 


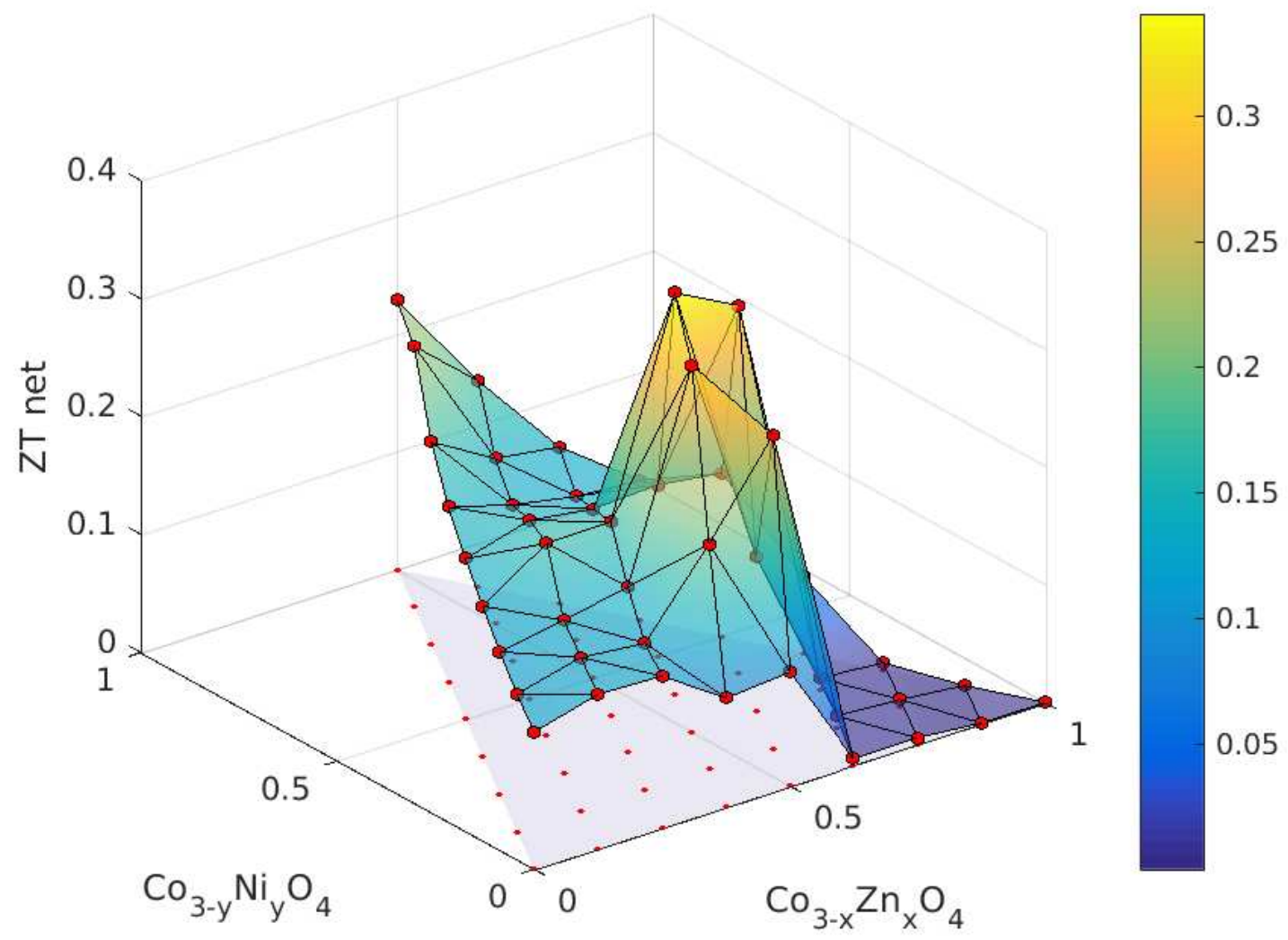

Figure 5.23 Spin Figure of merit of the configuraion space. Including the inverse spin Hall voltage, that is predominant in $\mathrm{Ni}$ substitution improved the ZT of $\mathrm{Ni}$ from 0.05 to 0.3 , which is almost 1 fold increase in ZT. 


\section{CHAPTER 6}

\section{CONCLUSIONS}

This thesis focused on development of the 1-D non-equilibrium Green's function for spin thermoelectric materials. This research presented a unique way to improve the ZT of the thermoelectric material by combining conventional charge voltage with additional spin voltage generated in a spin polarized material. The real contribution of this thesis was the development of a spin transport model that can be used to explore the design space spin based thermoelectric materials. To aid in increasing the autonomy of this model it was the goal of this research to also develop a method of deriving material properties from first principle density functional theory calculations. The developed method was validated against availble spin transverse device results and provided projections into the applicability of a new colbalt containing spinel design space for spin based thermoelectrics.

The inverse spin Hall voltage improves the overall performance of spin-polarized thermoelectric materials. Apt thermoelectric materials are neither metals nor insulators but possess semiconducting nature with high effective mass. Though effective mass has a less impact on conventional thermoelectric properties, it largely improves the inverse spin Hall voltage in the magnetic material thereby improving the performance of TE by many folds.

Validation of the model was performed using La:YIG garnites with the available experimental data. Using the similar approach, the model was applied to cobalt spinel oxides. The spin-polarization increases with Ni substitution into the material lattice and show more promising scope as spin based thermoelectrics. Theoretical calculations demonstrated a ten folds increase in ZT can be realized the combined contribution of both spin and conventional 
approaches. The compositional space formed by the end points of cobalt spinels $\mathrm{Co}_{3} \mathrm{O}_{4}$, $\mathrm{Co}_{2} \mathrm{ZnO}_{4}$ and $\mathrm{Co}_{2} \mathrm{NiO}_{4}$ was studied for spin based thermoelectric performance. $\mathrm{Ni}$ and $\mathrm{Zn}$ substitutions into the $\mathrm{Co}_{3} \mathrm{O}_{4}$ spinel matrix improved the spin caloritronic capability. It was observed that the focus region at $\mathrm{Co}_{2} \mathrm{Ni}_{0.25} \mathrm{Zn}_{0.75} \mathrm{O}_{4}$ showed a high increase in the overall $\mathrm{ZT}$ from 0.001 to 0.066 , hence providing a possible solution to the existing problem of low ZT in the current thermoelectric materials.

While this thesis focused on energy conversion the applications of spin based energy conversion is not restricted to energy generation but can also be used for data transfer in electronic devices. The model described in this research can be applied to study spin based transistors, spin based memory, and a wide variety of spin polarized materials in different applications. As this thesis presented the trend of increasing ZT by incorporating inverse spin Hall effect, more rigourous treatment of lattice thermal conductivity can yield reliable data for specific semiconducting thermoelectric materials.

The developed model is applicable to materials having a band gap between $0.07 \mathrm{eV}$ to $0.7 \mathrm{eV}$. Having near metallic nature with less than $0.07 \mathrm{eV}$, the model yields a low Seebeck coefficient that is close to $0 \mu \mathrm{V} / \mathrm{K}$. While having a band gap more than $0.7 \mathrm{eV}$, the modely gives negligible electrical conductivity thereby making it not effective for insulating materials. Semiconducting magnetic materials can be studied with the developed model.

Spin thermoelectric materials have immense scope for future energy applications and this thesis provides a proposed a possible model to perform theoretical study on a various semiconducting materials. 


\section{BIBLIOGRAPHY}

[1] Allon I. Hochbaum, Renkun Chen, Raul Diaz Delgado, Wenjie Liang, Erik C. Garnett, Mark Najarian, Arun Majumdar, and Peidong Yang. Enhanced thermoelectric performance of rough silicon nanowires. Nature, 451:163-167, Jan 2008.

[2] George S. Nolas, Joe Poon, and Mercouri Kanatzidis. Recent developments in bulk thermoelectric materials. MRS Bulletin, 31(3):199-205, 32006.

[3] H. Bottner, G. Chen, and R. Venkatasubramanian. Aspects of thin-film superlattice thermoelectric materials, devices and applications. MRS Bulletin, 31(3):211217, 2006.

[4] Terry M. Tritt and M. A. Subramanian. Thermoelectric materials, phenomena, and applications: A birds eye view. MRS Bulletin, 31(3):188198, Mar 2006.

[5] Patrick Gelsinger. Hot chips: Managing moores law. web source: news.utexas.edu, page last accessed 01/31/2017, Apr 2015.

[6] Tzu-Chen Hung, TY Shai, and S Kong Wang. A review of organic rankine cycles (orcs) for the recovery of low-grade waste heat. Energy, 22(7):661-667, 1997.

[7] Bahaa Saleh, Gerald Koglbauer, Martin Wendland, and Johann Fischer. Working fluids for low-temperature organic rankine cycles. Energy, 32(7):1210-1221, 2007.

[8] Edward Leo Murphy and RH Good Jr. Thermionic emission, field emission, and the transition region. Physical review, 102(6):1464, 1956.

[9] FA Padovani and R Stratton. Field and thermionic-field emission in schottky barriers. Solid-State Electronics, 9(7):695-707, 1966.

[10] Joseph P. Heremans, Mildred S. Dresselhaus, Lon E. Bell, and Donald T. Morelli. When thermoelectrics reached the nanoscale. Nat Nano, 8:471-473, July 2013. 
[11] Sun power datasheet x-21 335 residential solar panels.

[12] S. Manju and Netramani Sagar. Progressing towards the development of sustainable energy: A critical review on the current status, applications, developmental barriers and prospects of solar photovoltaic systems in india. Renewable and Sustainable Energy Reviews, 70:298 - 313, 2017.

[13] Daniel Kraemer, Bed Poudel, Hsien-Ping Feng, J. Christopher Caylor, Bo Yu, Xiao Yan, Yi Ma, Xiaowei Wang, Dezhi Wang, Andrew Muto, Kenneth McEnaney, Matteo Chiesa, Zhifeng Ren, and Gang Chen. High-performance flat-panel solar thermoelectric generators with high thermal concentration. Nat Mater, 10:532-538, 072011.

[14] P. Rojas Jhonathan, Singh Devendra, B. Inayat Salman, A. Torres Sevilla Galo, M. Fahad Hossain, and M. Hussain Muhammad. Reviewmicro and nano-engineering enabled new generation of thermoelectric generator devices and applications. ECS Journal of Solid State Science and Technology, 6:N3036-N3044, 2017.

[15] Photovoltaic (pv) pricing trends: Historical, recent, and near-term projections. Sunshot, Nov 2012.

[16] G. Jeffrey Snyder and Eric S. Toberer. Complex thermoelectric materials. Nat Mater, 7, 2008.

[17] Antoine Dewandre, Olle Hellman, Sandip Bhattacharya, Aldo H. Romero, Georg K. H. Madsen, and Matthieu J. Verstraete. Two-step phase transition in snse and the origins of its high power factor from first principles. Phys. Rev. Lett., 117:276601, Dec 2016.

[18] Gyung-Min Choi, Chul-Hyun Moon, Byoung-Chul Min, Kyung-Jin Lee, and David G. Cahill. Thermal spin-transfer torque driven by the spin-dependent seebeck effect in metallic spin-valves. Nature Physics, 11:576-581, 072015.

[19] D. M. Rowe. Crc handbook of thermoelectrics. 1995. 
[20] Zhi-Gang Chen, Guang Han, Lei Yang, Lina Cheng, and Jin Zou. Nanostructured thermoelectric materials: Current research and future challenge. Progress in Natural Science: Materials International, 22(6):535 - 549, 2012.

[21] Y. Nishino, S. Deguchi, and U. Mizutani. Thermal and transport properties of the heusler-type $\mathrm{fe}_{2} \mathrm{val}_{1-x} \mathrm{ge}_{x}(0 \leq \mathrm{x} \leq 0.20)$ alloys: Effect of doping on lattice thermal conductivity, electrical resistivity, and seebeck coefficient. Phys. Rev. B, 74:115115, Sep 2006.

[22] R Kitawaki and I Terasaki. Anomalous pd substitution effects in the thermoelectric oxide naco2xpdxo4. Journal of Physics: Condensed Matter, 14(47):12495, 2002.

[23] M A Hayward and R J Cava. The effect of elemental substitution on the electronic properties of ru2ge3. Journal of Physics: Condensed Matter, 14(25):6543, 2002.

[24] Wang Hong-Chao, Wang Chun-Lei, Zhang Jia-Liang, Zhao Ming-Lei, Liu Jian, $\mathrm{Su}$ Wen-Bin, Yin Na, and Mei Liang-Mo. Cu doping effect on electrical resistivity and seebeck coefficient of perovskite-type lafeo 3 ceramics. Chinese Physics Letters, 26(10):107301, 2009.

[25] H.C. Wang, C.L. Wang, W.B. Su, J. Liu, H. Peng, J.L. Zhang, M.L. Zhao, J.C. Li, N. Yin, and L.M. Mei. Substitution effect on the thermoelectric properties of reduced nb-doped sr0.95la0.05tio3 ceramics. Journal of Alloys and Compounds, 486(12):693 696, 2009.

[26] Haoshan Hao, Huizhi Yang, Yongtao Liu, and Xing Hu. High-temperature thermoelectric properties of cu-substituted bi2ba2co2-xcuxoy oxides. Journal of Materials Science \& Technology, 27(6):525 - 528, 2011.

[27] Yuichi Terazawa, Masashi Mikami, Takashi Itoh, and Tsunehiro Takeuchi. Effects of heavy element substitution on electronic structure and lattice thermal conductivity of fe2val thermoelectric material. Journal of Electronic Materials, 41(6):1348-1353, 2012. 
[28] Eric S. Toberer, M. Christensen, B. B. Iversen, and G. Jeffrey Snyder. High temperature thermoelectric efficiency in basga ${ }_{16} \mathrm{ge}_{30}$. Phys. Rev. B, 77:075203, Feb 2008.

[29] S. LeBlanc, S. K. Yee, M. L. Scullin, C. Dames, and K. E. Goodson. Material and manufacturing cost considerations for thermoelectrics. Renewable and Sustainable Energy Reviews, 32:313-327, 2014.

[30] Qing Jie, Hengzhi Wang, Weishu Liu, Hui Wang, Gang Chen, and Zhifeng Ren. Fast phase formation of double-filled p-type skutterudites by ball-milling and hot-pressing. Phys. Chem. Chem. Phys., 15:6809-6816, 2013.

[31] Glenn C. Catlin, Rajesh Tripathi, Geoffrey Nunes Jr., Philip B. Lynch, Howard D. Jones, and Devin C. Schmitt. An additive approach to low temperature zero pressure sintering of bismuth antimony telluride thermoelectric materials. Journal of Power Sources, 343:316 - 321, 2017.

[32] T. Okuda, K. Nakanishi, S. Miyasaka, and Y. Tokura. Large thermoelectric response of metallic perovskites: $\mathrm{sr}_{1-x} \mathrm{la}_{x} \mathrm{tio}_{3}(0 \& l t ; x \& l t ; 0.1)$. Phys. Rev. B, 63:113104, Mar 2001.

[33] Wolfgang G. Zeier, Jennifer Schmitt, Geoffroy Hautier, Umut Aydemir, Zachary M. Gibbs, Claudia Felser, and G. Jeffrey Snyder. Engineering half-heusler thermoelectric materials using zintl chemistry. Nature Reviews Materials, 50(4):16032 EP, 2016.

[34] Tian Yang, Bo Zhang, Ping Luo, Qing Zhao, Donglin He, and Aimin Chang. New ntc thermistors based on lacro3-mg(al0.7cr0.3)204 composite ceramics. Journal of Materials Science: Materials in Electronics, pages 1-4, 2017.

[35] Kei Hayashi, Tomohiro Nozaki, and Tsuyoshi Kajitani. Structure and high temperature thermoelectric properties of delafossite-type oxide cufe 1- x ni x o $2(0$ x 0.05). Japanese Journal of Applied Physics, 46(8R):5226, 2007. 
[36] Li-Dong Zhao, Shih-Han Lo, Yongsheng Zhang, Hui Sun, Gangjian Tan, Ctirad Uher, C. Wolverton, Vinayak P. Dravid, and Mercouri G. Kanatzidis. Ultralow thermal conductivity and high thermoelectric figure of merit in snse crystals. Nature, 508(7496):373377, 042014.

[37] G. S. Nolas, M. Kaeser, R. T. Littleton IV, and T. M. Tritt. High figure of merit in partially filled ytterbium skutterudite materials. Applied Physics Letters, 77(12):1855$1857,2000$.

[38] Tao He, Jiazhong Chen, H. David Rosenfeld, and M. A. Subramanian. Thermoelectric properties of indium-filled skutterudites. Chemistry of Materials, 18(3):759-762, 2006.

[39] Xun Shi, Jiong Yang, James R. Salvador, Miaofang Chi, Jung Y. Cho, Hsin Wang, Shengqiang Bai, Jihui Yang, Wenqing Zhang, and Lidong Chen. Multiple-filled skutterudites: High thermoelectric figure of merit through separately optimizing electrical and thermal transports. Journal of the American Chemical Society, 133(20):7837-7846, 2011. PMID: 21524125.

[40] G. Rogl, A. Grytsiv, P. Heinrich, E. Bauer, P. Kumar, N. Peranio, O. Eibl, J. Horky, M. Zehetbauer, and P. Rogl. New bulk p-type skutterudites dd0.7fe2.7co1.3sb12xxx $(\mathrm{x}=\mathrm{ge}, \mathrm{sn})$ reaching $\{\mathrm{ZT}\}$ \&gt; 1.3. Acta Materialia, 91:227 - 238, 2015.

[41] Duck-Young Chung, Tim Hogan, Paul Brazis, Melissa Rocci-Lane, Carl Kannewurf, Marina Bastea, Ctirad Uher, and Mercouri G. Kanatzidis. Csbi4te6: A highperformance thermoelectric material for low-temperature applications. Science, 287(5455):1024-1027, 2000.

[42] Bed Poudel, Qing Hao, Yi Ma, Yucheng Lan, Austin Minnich, Bo Yu, Xiao Yan, Dezhi Wang, Andrew Muto, Daryoosh Vashaee, Xiaoyuan Chen, Junming Liu, Mildred S. Dresselhaus, Gang Chen, and Zhifeng Ren. High-thermoelectric performance of nanostructured bismuth antimony telluride bulk alloys. Science, 320(5876):634-638, 2008. 
[43] L. Bocher, M. H. Aguirre, D. Logvinovich, A. Shkabko, R. Robert, M. Trottmann, and A. Weidenkaff. Camn1x nbxo3 (x 0.08) perovskite-type phases as promising new hightemperature n-type thermoelectric materials. Inorganic Chemistry, 47(18):8077-8085, 2008. PMID: 18698764.

[44] T. C. Harman, P. J. Taylor, D. L. Spears, and M. P. Walsh. Pbte-based quantum-dot thermoelectric materials with high zt. In Thermoelectrics, 1999. Eighteenth International Conference on, pages 280-284, Aug 1999.

[45] John Androulakis, Chia-Her Lin, Hun-Jin Kong, Ctirad Uher, Chun-I Wu, Timothy Hogan, Bruce A. Cook, Thierry Caillat, Konstantinos M. Paraskevopoulos, and Mercouri G. Kanatzidis. Spinodal decomposition and nucleation and growth as a means to bulk nanostructured thermoelectrics: enhanced performance in pb1-xsnxtepbs. Journal of the American Chemical Society, 129(31):9780-9788, 2007. PMID: 17629270.

[46] X. W. Wang, H. Lee, Y. C. Lan, G. H. Zhu, G. Joshi, D. Z. Wang, J. Yang, A. J. Muto, M. Y. Tang, J. Klatsky, S. Song, M. S. Dresselhaus, G. Chen, and Z. F. Ren. Enhanced thermoelectric figure of merit in nanostructured n-type silicon germanium bulk alloy. Applied Physics Letters, 93(19):193121, 2008.

[47] Wei-Shu Liu, Qinyong Zhang, Yucheng Lan, Shuo Chen, Xiao Yan, Qian Zhang, Hui Wang, Dezhi Wang, Gang Chen, and Zhifeng Ren. Thermoelectric property studies on cu-doped n-type cuxbi2te2.7se0.3 nanocomposites. Advanced Energy Materials, 1(4):577-587, 2011.

[48] Rutvik J. Mehta, Yanliang Zhang, Chinnathambi Karthik, Binay Singh, Richard W. Siegel, Theodorian Borca-Tasciuc, and Ganpati Ramanath. A new class of doped nanobulk high-figure-of-merit thermoelectrics by scalable bottom-up assembly. Nature Materials, 11(3):233-240, 2012. 
[49] Huili Liu, Xun Shi, Fangfang Xu, Linlin Zhang, Wenqing Zhang, Lidong Chen, Qiang Li, Ctirad Uher, Tristan Day, and G. Jeffrey Snyder. Copper ion liquid-like thermoelectrics. Nature Materials, 11(5):422-425, 2012.

[50] Paulo Carvillo, Yun Chen, Cullen Boyle, Paul N. Barnes, and Xueyan Song. Thermoelectric performance enhancement of calcium cobaltite through barium grain boundary segregation. Inorganic Chemistry, 54(18):9027-9032, 2015. PMID: 26357956.

[51] Steven N. Girard, Jiaqing He, Xiaoyuan Zhou, Daniel Shoemaker, Christopher M. Jaworski, Ctirad Uher, Vinayak P. Dravid, Joseph P. Heremans, and Mercouri G. Kanatzidis. High performance na-doped pbtepbs thermoelectric materials: Electronic density of states modification and shape-controlled nanostructures. Journal of the American Chemical Society, 133(41):16588-16597, 2011. PMID: 21902270.

[52] Qinyong Zhang, Hui Wang, Weishu Liu, Hengzhi Wang, Bo Yu, Qian Zhang, Zhiting Tian, George Ni, Sangyeop Lee, Keivan Esfarjani, Gang Chen, and Zhifeng Ren. Enhancement of thermoelectric figure-of-merit by resonant states of aluminium doping in lead selenide. Energy Environ. Sci., 5:5246-5251, 2012.

[53] Hui Zhang, Horst Borrmann, Niels Oeschler, Christophe Candolfi, Walter Schnelle, Marcus Schmidt, Ulrich Burkhardt, Michael Baitinger, Jing-Tai Zhao, and Yuri Grin. Atomic interactions in the p-type clathrate i ba8au5.3ge40.7. Inorganic Chemistry, 50(4):1250-1257, 2011. PMID: 21250680.

[54] Y. Saiga, B. Du, S.K. Deng, K. Kajisa, and T. Takabatake. Thermoelectric properties of type-viii clathrate ba8ga16sn30 doped with cu. Journal of Alloys and Compounds, $537: 303-307,2012$.

[55] Eyob K. Chere, Qian Zhang, Keshab Dahal, Feng Cao, Jun Mao, and Zhifeng Ren. Studies on thermoelectric figure of merit of na-doped p-type polycrystalline snse. $J$. Mater. Chem. A, 4:1848-1854, 2016. 
[56] Energy DOE. Thermoelectric materials devices and systems technology assessment. pages https://energy.gov/sites/prod/files/2015/02/f19/QTRic

[57] Seshadri Group. Energy materials datamining. UC Santa Barbara Materials Research Laboratory, Retrieved October 21, 2014:www.mrl.ucsb.edu:8080/datamine/thermoelectric.jsp, 2013.

[58] Gerrit E. W. Bauer, Eiji Saitoh, and Bart J. van Wees. Spin caloritronics. Nat Mater, 11, 2012.

[59] Li-Dong Zhao, Bo-Ping Zhang, Jing-Feng Li, Min Zhou, Wei-Shu Liu, and Jing Liu. Thermoelectric and mechanical properties of nano-sic-dispersed bi2te3 fabricated by mechanical alloying and spark plasma sintering. Journal of Alloys and Compounds, 455(1):259 - 264, 2008.

[60] Li-Dong Zhao, Vinayak P. Dravid, and Mercouri G. Kanatzidis. The panoscopic approach to high performance thermoelectrics. Energy Environ. Sci., 7:251-268, 2014.

[61] Yanzhong Pei, Xiaoya Shi, Aaron LaLonde, Heng Wang, Lidong Chen, and G. Jeffrey Snyder. Convergence of electronic bands for high performance bulk thermoelectrics. Nature, 473, 2011.

[62] Zhi-Gang Chen, Guang Han, Lei Yang, Lina Cheng, and Jin Zou. Nanostructured thermoelectric materials: Current research and future challenge. Progress in Natural Science: Materials International, 22(6):535 - 549, 2012.

[63] S. A. Wolf, D. D. Awschalom, R. A. Buhrman, J. M. Daughton, S. von Molnr, M. L. Roukes, A. Y. Chtchelkanova, and D. M. Treger. Spintronics: A spin-based electronics vision for the future. Science, 294(5546):1488-1495, 2001.

[64] Stephen R. Boona, Roberto C. Myers, and Joseph P. Heremans. Spin caloritronics. Energy Environ. Sci., 7:885-910, 2014. 
[65] K. Uchida, S. Takahashi, K. Harii, J. Ieda, W. Koshibae, K. Ando, S. Maekawa, and E. Saitoh. Observation of the spin seebeck effect. Nature Letters, 455:778-781, 2008.

[66] C. M. Jaworski, J. Yang, S. Mack, D. D. Awschalom, J. P. Heremans, and R. C. Myers. Observation of the spin-seebeck effect in a ferromagnetic semiconductor. Nat Mater, 9, Nov 2010.

[67] K. Uchida, J. Xiao, H. Adachi, J. Ohe, S. Takahashi, J. Ieda, T. Ota, Y. Kajiwara, H. Umezawa, H. Kawai, G. E. W. Bauer, S. Maekawa, and E. Saitoh. Spin seebeck insulator. Nat Mater, 9, Nov 2010.

[68] T. J. Seebek. Repts. Prussian Academy. Sci., 1823.

[69] J.C. Slonczewski. Current-driven excitation of magnetic multilayers. Journal of Magnetism and Magnetic Materials, 159(1):L1 - L7, 1996.

[70] G. Rogl and P. Rogl. Skutterudites, a most promising group of thermoelectric materials. Current Opinion in Green and Sustainable Chemistry, pages -, 2017.

[71] NK Appandairajan and J Gopalakrishnan. A study of co3xnixo4 (ox1) system. Proceedings of the Indian Academy of Sciences - Section A, Chemical Sciences, 87(4):115-120, 1978.

[72] J. Gopalakrishnan, N. K. Appandairajan, and B. Viswanathan. Co 3-x zn x o4 (0زxi1) spinel oxides. Proc. Indian Acad. Sci., 88 A:217 - 222, June 1979.

[73] Arthur J. Esswein, Meredith J. McMurdo, Phillip N. Ross, Alexis T. Bell, and T. Don Tilley. Size-dependent activity of co3o4 nanoparticle anodes for alkaline water electrolysis. The Journal of Physical Chemistry C, 113(33):15068-15072, 2009.

[74] Paul F. Ndione, Andres Garcia, N. Edwin Widjonarko, Ajaya K. Sigdel, K. Xerxes Steirer, Dana C. Olson, Philip A. Parilla, David S. Ginley, Neal R. Armstong, Robin E. Richards, Erin L. Ratcliff, and Joseph J. Berry. Highly-tunable nickel cobalt oxide as 
a low-temperature p-type contact in organic photovoltaic devices. Advanced Energy Materials, 3(4):524-531, 2013.

[75] Charles F. Windisch, Kim F. Ferris, and Gregory J. Exarhos. Synthesis and characterization of transparent conducting oxide cobaltnickel spinel films. Journal of Vacuum Science \& Technology A, 19(4):1647-1651, 2001.

[76] Florin Tudorache, Paul Dorin Popa, Marius Dobromir, and Felicia Iacomi. Studies on the structure and gas sensing properties of nickelcobalt ferrite thin films prepared by spin coating. Materials Science and Engineering: B, 178(19):1334 - 1338, 2013. Selected Papers from the 9th International Conference on Physics of Advanced Materials (ICPAM9).

[77] Yan Zhang, Jonathan Rosen, Gregory S. Hutchings, and Feng Jiao. Enhancing photocatalytic oxygen evolution activity of cobalt-based spinel nanoparticles. Catalysis Today, 225(0):171 - 176, 2014. Recent Progresses in the Area of Photocatalysis Research.

[78] N. Bahlawane*, P. A. Premkumar, J. Feldmann, and K. Kohse-Hinghaus. Preparation of doped spinel cobalt oxide thin films and evaluation of their thermal stability. Chemical Vapor Deposition, 13(2-3):118-122, 2007.

[79] Makhtar Guene, Abdou Aziz Diagne, Modou Fall, Mor Marme Dieng, and Grard Poillerat. Preparation of nickel - cobalt spinel oxides $\mathrm{ni}_{3-x} \mathrm{O}_{4}$. comparison of two physical properties stemming from four different preparation methods and using carbon paste electrode. Bulletin of the Chemical Society of Ethiopia, 21(2):255-262, 2007.

[80] N.K. Appandairajan, B.Viswanathan, and J.Gopalakrishnan. Magnetic-properties of nixco3-xo4 system. Revue Roumaine de Physique, 25(2):207 - 208, 1980.

[81] Donald S. McClure. The distribution of transition metal cations in spinels. Journal of Physics and Chemistry of Solids, 3(34):311 - 317, 1957. 
[82] Junichi Takaobushi, Mizue Ishikawa, Shigenori Ueda, Eiji Ikenaga, Jung-Jin Kim, Masaaki Kobata, Yukiharu Takeda, Yuji Saitoh, Makina Yabashi, Yoshinori Nishino, Daigo Miwa, Kenji Tamasaku, Tetsuya Ishikawa, Issei Satoh, Hidekazu Tanaka, Keisuke Kobayashi, and Tomoji Kawai. Electronic structures of $\mathrm{fe}_{3-x} \mathrm{~m}_{x} \mathrm{O}_{4}(\mathrm{~m}=\mathrm{mn}, \mathrm{zn})$ spinel oxide thin films investigated by x-ray photoemission spectroscopy and $\mathrm{x}$-ray magnetic circular dichroism. Phys. Rev. B, 76:205108, Nov 2007.

[83] Nicola H. Perry, Thomas O. Mason, Chengcheng Ma, Alexandra Navrotsky, Yezhou Shi, Joanna S. Bettinger, Michael F. Toney, Tula R. Paudel, Stephan Lany, and Alex Zunger. Co3o4co2zno4 spinels: The case for a solid solution. Journal of Solid State Chemistry, 190(0):143 - 149, 2012.

[84] Supriyo Datta. Nanoscale device modeling: the greens function method. Superlattices and Microstructures, 28(4):253 - 278, 2000.

[85] Jiang Xiao, Gerrit E. W. Bauer, Ken-chi Uchida, Eiji Saitoh, and Sadamichi Maekawa. Theory of magnon-driven spin seebeck effect. Phys. Rev. B, 81:214418, Jun 2010.

[86] K Stokbro. First-principles modeling of electron transport. Journal of Physics: Condensed Matter, 20(6):064216, 2008.

[87] Po-Hao Chang, Farzad Mahfouzi, Naoto Nagaosa, and Branislav K. Nikolić. Spinseebeck effect on the surface of a topological insulator due to nonequilibrium spinpolarization parallel to the direction of thermally driven electronic transport. Phys. Rev. B, 89:195418, May 2014.

[88] Mads Brandbyge, José-Luis Mozos, Pablo Ordejón, Jeremy Taylor, and Kurt Stokbro. Density-functional method for nonequilibrium electron transport. Phys. Rev. B, 65:165401, Mar 2002.

[89] K. Uchida, T. Ota, K. Harii, S. Takahashi, S. Maekawa, Y. Fujikawa, and E. Saitoh. 
Spin-seebeck effects in ni81fe19/pt films. Solid State Communications, 150(11):524528, 2010. Spin Caloritronics.

[90] Xingtao Jia, Kai Liu, Ke Xia, and Gerrit E. W. Bauer. Spin transfer torque on magnetic insulators. EPL (Europhysics Letters), 96(1):17005, 2011.

[91] Yong-Nian Xu, Zong quan Gu, and W. Y. Ching. First-principles calculation of the electronic structure of yttrium iron garnety3fe5o12. Journal of Applied Physics, 87(9):4867-4869, 2000.

[92] Pio Baettig and Tamio Oguchi. Why are garnets not ferroelectric? a theoretical investigation of y3fe5o12. Chemistry of Materials, 20(24):7545-7550, 2008.

[93] W. Kohn and L. J. Sham. Self-consistent equations including exchange and correlation effects. Phys. Rev., 140:A1133-A1138, Nov 1965.

[94] P. Hohenberg and W. Kohn. Inhomogeneous electron gas. Phys. Rev., 136:B864-B871, Nov 1964.

[95] Paolo Giannozzi, Stefano Baroni, Nicola Bonini, Matteo Calandra, Roberto Car, Carlo Cavazzoni, Davide Ceresoli, Guido L Chiarotti, Matteo Cococcioni, Ismaila Dabo, Andrea Dal Corso, Stefano de Gironcoli, Stefano Fabris, Guido Fratesi, Ralph Gebauer, Uwe Gerstmann, Christos Gougoussis, Anton Kokalj, Michele Lazzeri, Layla MartinSamos, Nicola Marzari, Francesco Mauri, Riccardo Mazzarello, Stefano Paolini, Alfredo Pasquarello, Lorenzo Paulatto, Carlo Sbraccia, Sandro Scandolo, Gabriele Sclauzero, Ari P Seitsonen, Alexander Smogunov, Paolo Umari, and Renata M Wentzcovitch. Quantum espresso: a modular and open-source software project for quantum simulations of materials. Journal of Physics: Condensed Matter, 21(39):395502, 2009.

[96] John P. Perdew, Kieron Burke, and Yue Wang. Generalized gradient approximation for the exchange-correlation hole of a many-electron system. Phys. Rev. B, 54:1653316539, Dec 1996. 
[97] John P. Perdew, Kieron Burke, and Matthias Ernzerhof. Generalized gradient approximation made simple. Phys. Rev. Lett., 77:3865-3868, Oct 1996.

[98] David Vanderbilt. Soft self-consistent pseudopotentials in a generalized eigenvalue formalism. Phys. Rev. B, 41:7892-7895, Apr 1990.

[99] P. M. Marcus and V. L. Moruzzi. Stoner model of ferromagnetism and total-energy band theory. Phys. Rev. B, 38:6949-6953, Oct 1988.

[100] Jørn Foros, Arne Brataas, Yaroslav Tserkovnyak, and Gerrit E. W. Bauer. Magnetization noise in magnetoelectronic nanostructures. Phys. Rev. Lett., 95:016601, Jun 2005.

[101] Jiang Xiao, Gerrit E. W. Bauer, Sadamichi Maekawa, and Arne Brataas. Charge pumping and the colored thermal voltage noise in spin valves. Phys. Rev. B, 79:174415, May 2009.

[102] Yaroslav Tserkovnyak, Arne Brataas, and Gerrit E. W. Bauer. Enhanced gilbert damping in thin ferromagnetic films. Phys. Rev. Lett., 88:117601, Feb 2002.

[103] E. Saitoh, M. Ueda, H. Miyajima, and G. Tatara. Conversion of spin current into charge current at room temperature: Inverse spin-hall effect. Applied Physics Letters, 88(18):182509, 2006.

[104] Y. Kajiwara, K. Harii, S. Takahashi, J. Ohe, K. Uchida, M. Mizuguchi, H. Umezawa, H. Kawai, K. Ando, K. Takanashi, S. Maekawa, and E. Saitoh. Transmission of electrical signals by spin-wave interconversion in a magnetic insulator. Nature, 464, Mar 2010.

[105] T. Kimura, Y. Otani, T. Sato, S. Takahashi, and S. Maekawa. Room-temperature reversible spin hall effect. Phys. Rev. Lett., 98:156601, Apr 2007. 